\title{
Phenotype-dependent social environment choice in the male Trinidadian guppy
}

\author{
by
}

Sophie Joanna Potter

A thesis submitted to the Faculty of Graduate and Postdoctoral Affairs in partial fulfillment of the requirements for the degree of Doctor of Philosophy in

Biology

Carleton University Ottawa, Ontario

(C) 2019, Sophie Joanna Potter 


\section{Abstract}

Prey animals living under threat of predation must maximise their fitness by minimising their risk of predation using strategies such as altering behaviour and selecting the most beneficial social environment. I used the Trinidadian guppy (Poecilia reticulata) as a model study species to investigate the potential effects of predation and conspecific phenotype on male social and sexual behaviours. Based on field collections of wild guppies from river populations in Trinidad that vary in their overall level of predation risk, I found significant differences in average male phenotype (i.e. body length and body coloration) among the populations, with males from the lowest predation-risk population being larger whereas males from an intermediate-risk population (where water is turbid due to mining activity) were the least colourful. I found no difference in guppy shoaling behaviour among populations but did note some differences in sexual behaviour with males from the high-predation Quaré River more frequently performing sneak copulation attempts compared to males from less risky environments.

In the laboratory, I evaluated whether and how the phenotype of conspecific males affected the social association preferences of focal males. I found that focal males exhibited no shoaling preference for a specific male phenotype when presented with mixed-sex stimulus shoals. However, when females were absent, focal males preferred to associate with large, colourful males. As there was no significant difference among populations, I also tested male association preferences in the presence and absence of chemical alarm cues to ascertain whether a perceived immediate risk of predation would 
affect social choice. I found an overall preference for more attractive males in both the presence and absence of the alarm cue (with some exceptions), though males from the high-predation river moved between shoals less in the presence of an alarm cue, suggesting minimisation of visual conspicuousness. I also tested whether an increased male shoal size affected female social preference for more attractive males, but results did not support this. Overall, my work suggests that wild-caught Trinidadian male guppies prefer to socially associate with certain conspecific males that differ in phenotype from themselves, though the evolutionary function(s) of such preferences remain uncertain and require further investigation. 


\section{Acknowledgments}

Firstly, I would like to extend my greatest appreciation and thanks to my cosupervisors Jean-Guy Godin and Tom Sherratt for their help and patience throughout my Ph.D. Your support has been invaluable and I would not have made it through this process without you. I am also deeply thankful that you supported my attendance at several international and national conferences throughout the course of my studies. Additionally, I am grateful for the help and guidance of the other members of my thesis advisory committee, Sue Bertram and Julie Morand-Ferron, for their support and input throughout the course of my Ph.D.

Many members of the Godin and Sherratt labs have provided support, but special mention should go to Heather Auld for help in collecting data in Trinidad, and Ryan Pusiak, Anne-Christine Auge and Skye Litvack for their help in maintaining our guppy colony at Carleton University. Additionally, Anne-Christine allowed me use of her data set of Upper Aripo male body lengths and coloration scores, which was included in Chapter 3 of my thesis. Casey Peet-Paré produced the guppy cartoons which I have used both in this thesis and in many presentations of this work, which have always made explanations of my experiments much easier to visualise. Changku Kang provided support with statistical analysis, along with many morale-boosting baked treats from Yongmi Kim. Richard and Francina Webster gave me a place to stay when I first arrived and have been my cheerleaders and theatre buddies ever since. Lauren Efford, Ian Dewan, Felipe Dargent and Nina Kniel have been instrumental in supporting me through the writing process and 
kept me from losing perspective. Rhiannon Adler, Ebony Demers, Tammy Duong, Matthew Potter, Siân Rowland, Ais Wyatt and Kai Zarkow formed my team of proofreaders, and have been vital in helping me catch errors that had slipped into the thesis during the repeated editing process. I greatly appreciate their time and help, and all remaining errors are entirely my fault!

I thank Grant Brown, Ebony Demers and Laurence Feyten for their companionship during my research in Trinidad. Ebony also provided significant moral support during the writing of this thesis, for which I am incredibly grateful. Indar Ramnarine allowed me use of research lab facilities at the University of the West Indies, St Augustine, without which this work would not have been possible. I also thank Kharran Deonarinesingh for his vital assistance in collecting and maintaining the guppies in Trinidad.

Despite the ocean between us, I am grateful to my family for their support throughout this process. My parents Alan and Chris have always encouraged my education and my interest in the natural world, and it is thanks to them that I was able to complete this Ph.D. thesis. My brother Matthew has been a vital ally throughout my Ph.D. studies and especially during the writing phase, always reminding me that there is light at the end of the tunnel. My thanks as well go to Emma, for tolerating all of us and for always supporting me. During the course of my Ph.D., I was unfortunate enough to lose my beloved Grandmas, Dot Potter and Rita Green. Their nurturing love and belief in me will never be forgotten, and I dedicate this thesis to them both. 
So many friends have been vital in getting me through this thesis and it is impossible to name everyone. However, particular thanks go to Ais Wyatt for providing a supportive ear and many delicious dinners. I thank my bookstore family, Lekan Oyelolo and lan Krzyzanowski, for always cheering me on and making me laugh. Genevieve Ferguson also provided valuable support and morale-boosts as a recent Ph.D. graduate.

I am indebted to Eric Guerra-Grenier for his unwavering belief in me, and for the numerous games of squash which helped keep me active and unwound throughout this process. Finally, my thanks and gratitude to Tammy Duong, who made this Ph.D. such a wonderful experience, and whose love and encouragement I will treasure forever. 


\section{Table of Contents}

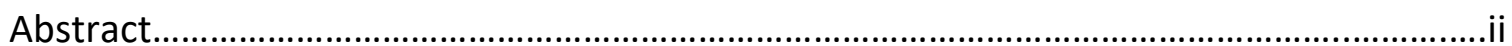

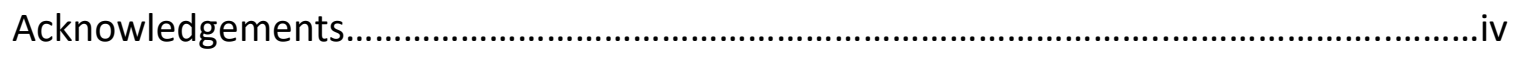

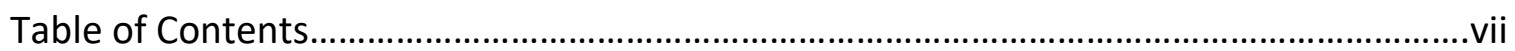

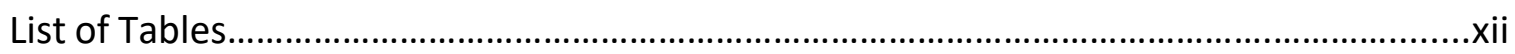

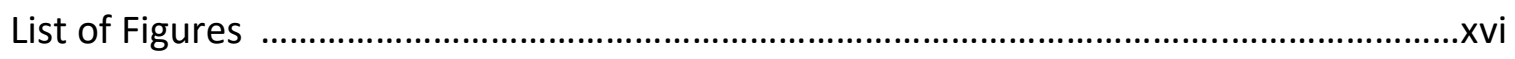

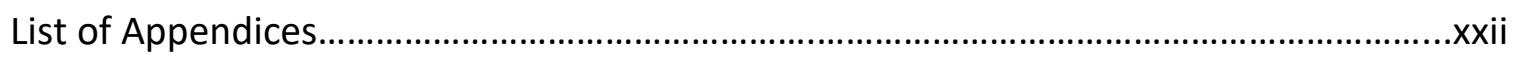

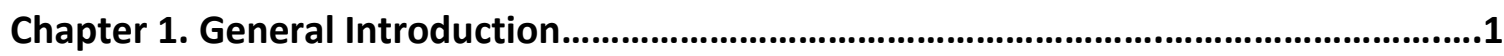

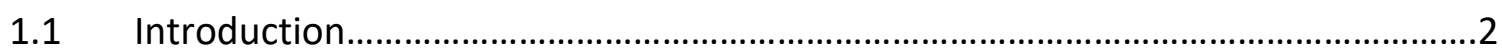

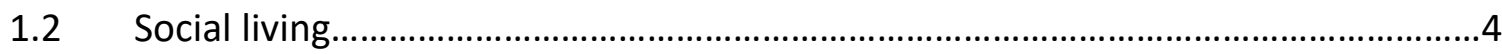

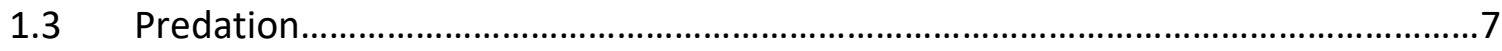

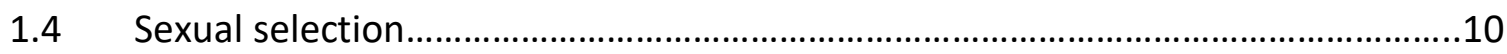

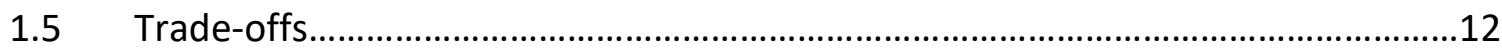

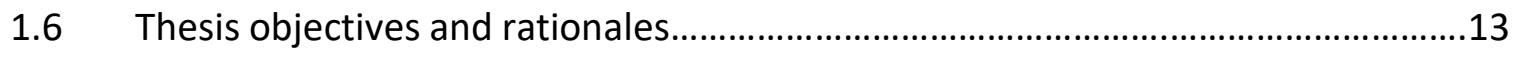

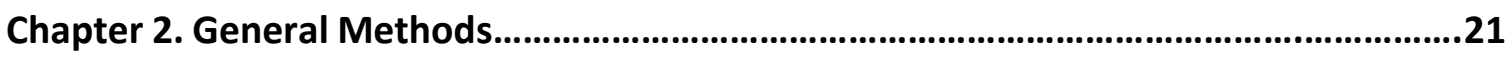

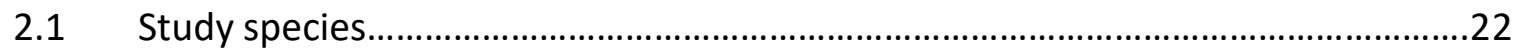

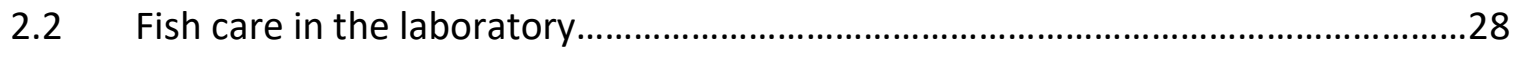


2.3 Dichotomous choice tests.

2.4 Measurements of fish body length and coloration

Chapter 3. Sex, lives, and videotape: A cross-population comparison of the social and sexual behaviours of free-ranging male Trinidadian guppies........................................34

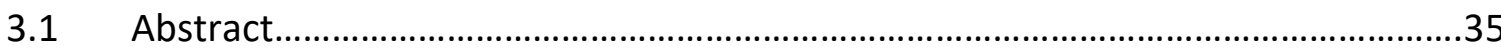

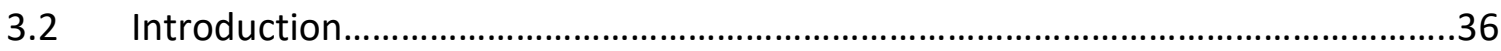

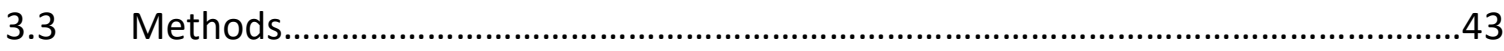



3.3.2 Video recording and quantification of male behaviour.............................45

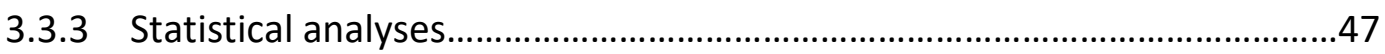

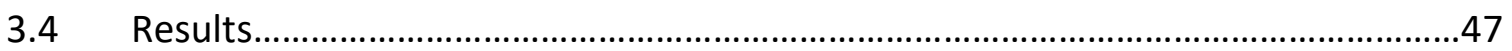

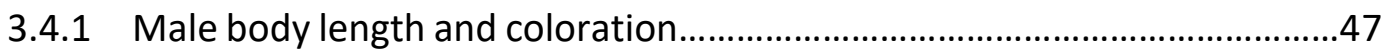

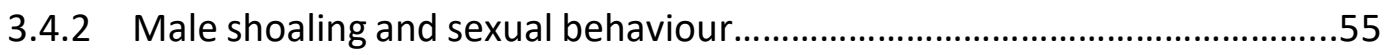

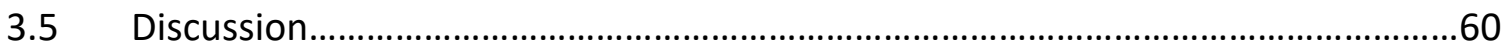

Chapter 4. Dangerous liaisons: Effects of females and population predation risk on male-male social associations in the Trinidadian guppy..................................................67

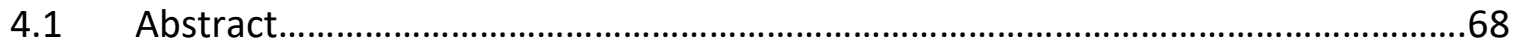

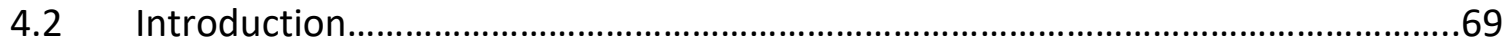




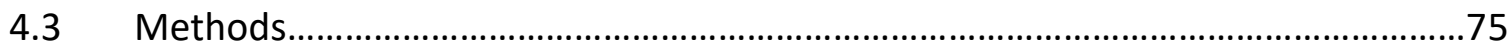

4.3.1 Fish collection and maintenance..............................................................

4.3.2 Experimental protocol and apparatus.........................................................76

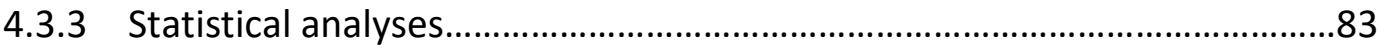

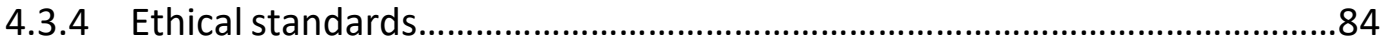

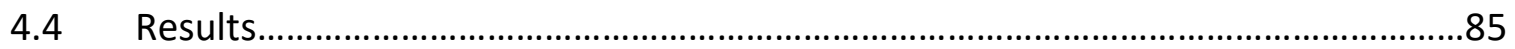

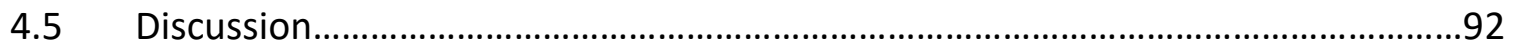

Chapter 5. Winning friends and influencing females: Does the number of available males alter female association preferences?.............................................................96

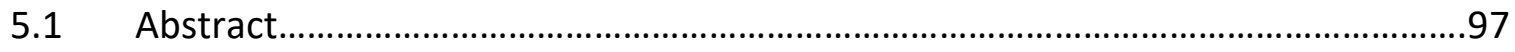

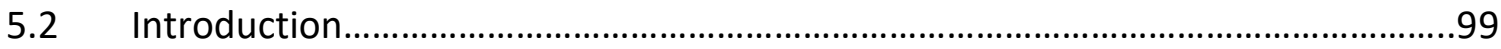

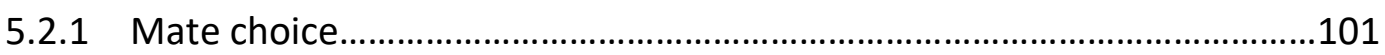

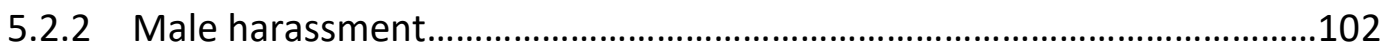

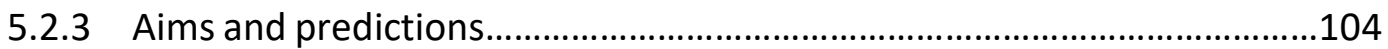

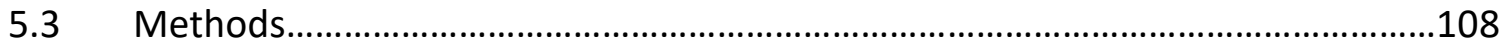

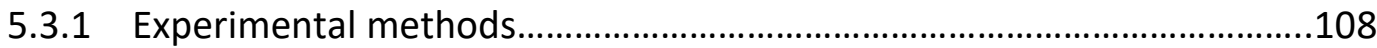

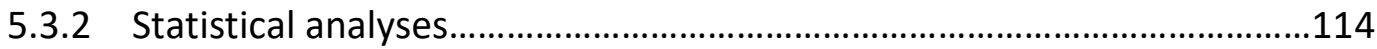

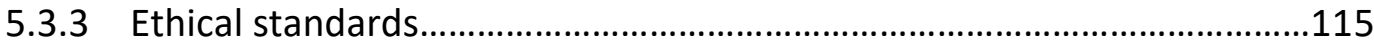


5.4 Results.

5.5 Discussion. .124

Chapter 6. With friends like these, who needs enemies? The effect of chemical alarm cues on male-male social environment choice.........................................................129

6.1 Abstract.

6.2 Introduction.

6.2.1 Group living and associative shoaling.

6.2.2 Population predation level.

6.2.3 Predation cues.

6.2.4 Aims and predictions

6.3 Methods

6.3.1 Fish collection and maintenance.

6.3.2 Experimental protocol and apparatus. .146

6.3.3 Statistical analyses.

6.3.4 Ethical standards

6.4 Results. .153

6.5 Discussion. .160

Chapter 7. General Discussion. .165 


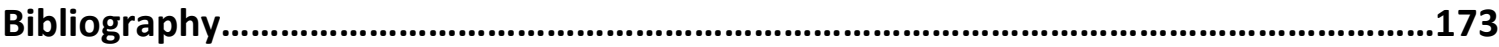

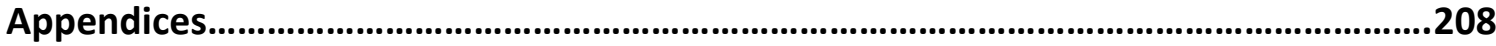

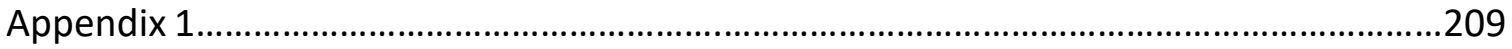

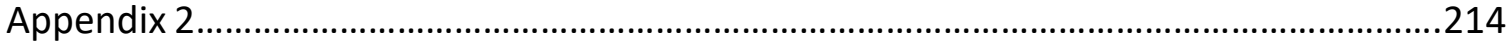

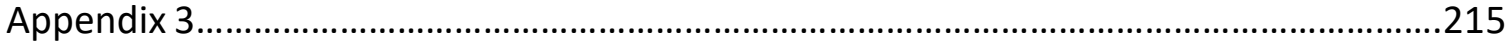

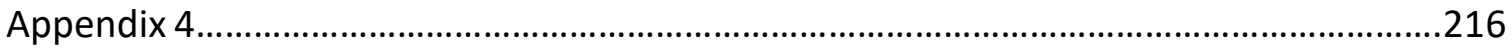

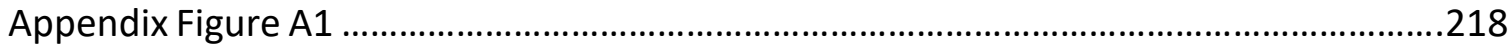

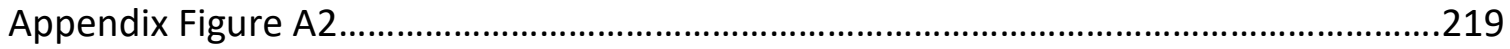




\section{List of Tables}

Table 3.1. Results of a post-hoc Dunn test comparing male guppy total body length data across the four study populations (Quaré: high-predation; Arima: intermediatepredation; Tunapuna \& Upper Aripo: low-predation) in a pairwise manner. Boldfaced pvalues are statistically significant.

Table 3.2. Results of a post-hoc Dunn test comparing male guppy body coloration scores across the four study populations (Quaré: high-predation; Arima: intermediatepredation; Tunapuna \& Upper Aripo: low-predation) in a pairwise manner. Boldfaced pvalues are statistically significant.

Table 3.3. Results of a post-hoc Dunn test comparing (a) the mean rate of focal male courtship displays and (b) the mean rate of focal male sneak copulation attempts across the three study populations (Quaré: high-predation; Tunapuna \& Upper Aripo: lowpredation) in a pairwise manner. Boldfaced $p$-values are statistically significant. .59

Table 4.1. Total number of trials (focal males) for each of the treatments and study populations (Quaré: high-predation; Arima: intermediate-predation; Tunapuna: lowpredation) 79

Table 4.2. Mean \pm SE total body length $(\mathrm{mm})$ and body colour score (proportion of left side covered with orange and black coloration) of focal males and stimulus males classified as more and less attractive stimulus fish. Each paired t-test compares the phenotypic measures for the paired more attractive and less attractive stimulus males used in individual trials .86 
Table 4.3 Results of paired t-tests comparing the body lengths of the two stimulus females (one in each of the paired stimulus shoals) presented during the female-present trials. As indicated by $p$ values of $>0.05$, there was no significant difference between the sets of females, suggesting female body length should not have had a significant effect on focal male shoal choice.

Table 4.4. Results of one-sample t-tests comparing the proportion of time the focal male spent shoaling with the more attractive shoal against the proportion $(=0.50)$ expected by chance (i.e. no preference). $\mathrm{P}$ values in boldface indicate significant differences, and therefore a preference for either stimulus shoal .90

Table 4.5. ANOVA table and parameter estimates of the general linear model testing for the effects of population, treatment (female presence/absence) and phenotypic traits (length and colour score of focal males, and the difference in the body length and colour score of the males in the paired stimulus shoals) on the time focal males spent associating with the more attractive stimulus shoal. The $P$ value in boldface indicates a significant treatment effect on the response variable. .91

Table 5.1 Number of replicates for each of the different sets of experimental conditions. .111

Table 5.2: Results of one-sample t-tests comparing the proportion of time the focal female spent near the sexually more attractive male(s) against that expected by chance (proportion $=0.50) . p$-values in boldface indicate significant differences and therefore a preference for the sexually more attractive males. .118 
Table 5.3 Results of paired t-tests comparing the proportion of time the focal female spent near the sexually more attractive male in Presentation 1 against the proportion of time spent near the sexually more attractive male(s) in Presentation 2 for each of the two treatments for both study populations. None of the p-values are statistically different.

Table 5.4. Results of significance testing dropping single predictors to determine important predictors in the fitted model: Proportion of time with MA Presentation Order + Population + Treatment + Length Difference + Colour Difference $+(1 \mid$ Female ID $)$. Treatment, presentation order and population were included as fixed factors. Values in boldface indicate significant effects.

Table 5.5. The fixed effects of model parameters for the fitted model: Proportion of time with MA Presentation Order + Population + Treatment + Length Difference + Colour Difference $+(1 \mid$ Female ID $)$

Table 6.1. Number of replicates for each of the different treatments used. Owing to inadvertent data recording errors, some of the shoal switches exhibited by the focal male in some of the trials were not noted and consequently the number of replicate trials shown below differed between the two behavioural measures. .143

Table 6.2. Mean \pm SE body length $(\mathrm{mm})$ and coloration score (proportion of body covered with patches of orange and black colours) for the more attractive shoal, focal males and less attractive shoal for each of the different conditions tested. .145 
Table 6.3: Results of Wilcoxon signed-rank tests comparing the proportion of total shoaling time focal males spent associating with the more attractive shoal against that expected by chance (i.e. proportion $=0.50$ ). Boldface $p$ values in indicate preferences for the more attractive shoal that are greater than expected by chance...... .155

Table 6.4. ANOVA table and parameter estimates of the general linear model testing for the effects of cue type (treatment), focal male type and population on the time focal males spent shoaling with the more attractive shoal. .157

Table 6.5. ANOVA table and parameter estimates of a general linear model testing for the effects of cue type, focal male type and population on the frequency of shoal switches performed by focal males. 


\section{List of Figures}

Figure 2.1 Top view of a schematic of the experimental apparatus, with measurements. Dotted lines indicate the boundary of the $10-\mathrm{cm}$ preference zone (marked beneath the tank using marker pen on Styrofoam) adjacent to each of the stimulus fish end compartments. The circle in the central test aquarium denotes a clear Plexiglas cylinder (7 cm diam.) that temporarily held the focal male guppy during the acclimatisation period prior to choice tests. Thick black lines indicate removable opaque Plexiglas screens that initially blocked the view of the focal male into the end stimulus fish compartments. The central test aquarium and end compartments were constructed of clear Plexiglas..........30

Figure 3.1. Mean \pm 1 SE adult guppy density for each of the four study populations $(n=10$ per population). Bar colour relates to predation level of the river (red= high predation, yellow $=$ intermediate, green $=$ low)

Figure 3.2. Mean \pm 1 SE proportion of females collected in seine samples within each of the four study populations ( $n=10$ per population). Bar colour corresponds to predation

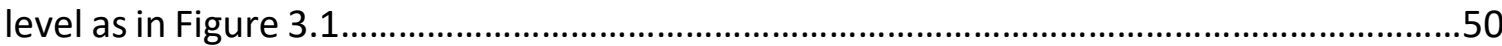

Figure 3.3. Density distribution of body length measures of free-ranging male guppies collected by seining in each of the four study populations ( $n=150$ males per population). Line colour indicates population predation level (as in Figure 3.1) .51

Figure 3.4. Density distribution of body coloration score (proportion of a male's body covered with orange and black colours) of free-ranging male guppies collected by seining 
in each of the four study populations ( $n=150$ males per population). Line colour indicates population predation level (as in Figure 3.1) 53

Figure 3.5. Box plots (showing median and inter-quartile intervals, with dots indicating outliers) of the shoaling behaviour metrics recorded from video clips of focal males across three populations (Quaré: high-predation; Tunapuna \& Upper Aripo: low-predation). a) Amount of time focal males spent shoaling (within 4 body lengths of a conspecific) as a percentage of total observation time for individual shoals. b) Rate at which a focal male entered new shoals per minute of total observation time (= shoal encounter rate). c) Shoal tenure duration (time that focal male remained in a shoal) in seconds. d) Maximum number of individuals in a shoal containing a focal male. .56

Figure 3.6. Box plots (showing the median and inter-quartile intervals, with dots indicating outliers) of the rate of sexual behaviours of focal males recorded during video analysis across three study populations (Quaré: high-predation; Tunapuna \& Upper Aripo: lowpredation). a) Number of courtship displays performed by focal males per minute spent shoaling, and b) Number of sneak copulation attempts performed by focal males per minute spent shoaling. .58

Figure 4.1. Composition of the paired stimulus shoals for a) the female present and b) female absent experimental treatments. Each focal male was only subjected to one of the two treatments. .78

Figure 4.2: Schematic of the experimental apparatus (overhead view) showing the experimental tanks for a social association preference test. The middle aquarium contains 
the focal male (FM), held initially within a clear tube (shown), whilst the two end containers each hold a stimulus shoal of two fish separated by a clear divider. Here, the more attractive male (MAM) is on the left side, and the less attractive male (LAM) on the right. This figure illustrates the set-up for the female-present treatment, where two sizematched females are placed one at either end. For the female-absent treatment, the females would be replaced by a second stimulus male (of a similar phenotype to the other male in the relevant stimulus shoal) taken from the same population as the focal male. The apparatus is pictured here with the opaque screens in place, blocking the stimulus shoals from the focal male's view. These screens were removed during the acclimatisation period, prior to the start of the free-swimming choice period (as described below).............. .81

Figure 4.3. Mean \pm SE proportion of time focal males spent associating (shoaling) with the shoal containing the more attractive male(s) across the three study populations (Quaré: high-predation; Arima: intermediate-predation; Tunapuna: low-predation), when females were present in the stimulus shoals (open bar) and when females were absent (filled bar). An asterisk $(*)$ above a mean denotes a choice preference for the more attractive shoal that is significantly greater than that expected from chance (indicated by the horizontal stippled line at 0.50 ; see details of the one-sample t-tests in Table 4.6). No significant different from chance is denoted by N.S. .89

Figure 5.1. The different stimulus males presented to individual focal female. Each female was presented with males twice. In Presentation 1, she was offered a choice between a single colourful male and a single drab male. In Presentation 2, she was assigned to one of two treatment groups: a) In Treatment 1 (the consistent stimulus group), Presentation 
2 consisted of the same two males as in Presentation 1. b) In Treatment 2 (the increased stimulus group), the focal female was also presented with the same males as in Presentation 1, but in this scenario they were each accompanied by two additional males of similar phenotype, meaning that the female was now being presented with two shoals of three males each. .106

Figure 5.2. Mean \pm SE proportion of time spent associating with the sexually more attractive (MA) stimulus males by focal females from the a) Tunapuna River (lowpredation) and b) Quaré River (high-predation) over the two presentations in each of the two treatments. Table 5.2 below gives full results of one-sample t-tests against 0.5 (no preference). An asterisk $\left({ }^{*}\right)$ above a mean denotes a choice preference for the MA shoal that is significantly greater than that expected from chance (indicated by the stippled line at 0.5 ) .117

Figure 5.3: Mean \pm SE difference in preference for the more attractive shoal on the second presentation of stimulus males, either as a consistent pair of males (Treatment 1- white bars) or as part of two larger shoals each containing 3 individuals (Treatment 2-shaded bars). Negative difference scores (as seen here) indicate a decrease in preference for the more attractive shoal. Tunapuna= low-predation, Quaré= high-predation.

Figure 6.1. The different male phenotypes used in each of the different trials for a given population. Focal males were classified as either a) attractive or b) drab, relative to the overall population, and were always presented with a choice between a shoal 
phenotypically (both length and coloration) similar to himself and another shoal phenotypically different from himself.

Figure 6.2. Overhead view of the experimental set-up, showing a central test arena and two smaller aquaria holding the male stimulus shoals at either end. In this diagram, a less attractive (LA) focal male (FM) is being offered a choice between a shoal of more attractive males (MAM) and a shoal of less attractive males (LAM). The central cylinder is perforated to allow exposure of the focal male to the chemical alarm cue or water cue in the central arena, depending on treatment. The open circle at the side and centre of the central arena indicates airline tubing where alarm cue or water is administered. The shaded circle is the airstone, which allows aeration of the water and distribution of the added substance. The dark thick lines denote removable opaque Plexiglas screens. The stippled lines denote a $10-\mathrm{cm}$ wide shoal preference zone in front of either stimulus shoals 149

Figure 6.3: Mean \pm SE proportion of total shoaling time focal males spent associating with the more attractive (MA) stimulus shoal by focal males from the (a) Tunapuna River (lowpredation) and (b) Quaré River (high-predation) in either the presence of a chemical alarm cue (shaded bars) or a water control (white bars). Table 6.4 gives results of Wilcoxon signed-rank tests comparing observed preference scores against that expected by chance (i.e. proportion $=0.50)$. An asterisk $(*)$ above a mean denotes a preference for the MA shoal that is significantly greater than expected from chance (indicated by the horizontal stippled line at 0.50) 154 
Figure 6.4. Mean \pm SE number of switches (movements from associating with one stimulus shoal to associating with the other shoal) in shoaling association by focal males from the (a) Tunapuna River (low-predation) and (b) Quaré River (high-predation) in either the presence of a chemical alarm cue (shaded bars) or a water control (white






\section{List of Appendices}

Appendix 1. Confirmation that discarding trials where significant $(>80 \%)$ side bias is observed does not significantly alter the type I error rate (including R code). .209

Appendix 2. Confirmation that the total time focal males were observed did not significantly differ among the 3 different populations recorded .214

Appendix 3. Results of paired t-tests comparing the mean time spent shoaling (within one of the two preference zones) by focal females in Presentation 1 and Presentation 2 for both populations tested (Tunapuna= low-predation; Quaré= high-predation). $\mathrm{p}$ values indicate that there was no significant difference in the amount of time spent shoaling in Presentation 1 and Presentation 2 for any treatment. .215

Appendix 4. Results of paired t-tests to test the null hypothesis that there was no significant difference in (a) body length or (b) coloration between the focal and stimulus males, and also between the two sets of stimulus males (more and less attractive). All significant values indicated in boldface. In all cases more and less attractive stimulus males were significantly different from one another, and focal males were significantly different from the stimulus males of the opposite type to themselves (i.e. a less attractive focal male was significantly different from the more attractive stimulus males but was not significantly different from the less attractive stimulus males).

Appendix Figure A1. Example of variation in body length and coloration in male guppies collected from the Quaré River, Trinidad. .218 
Appendix Figure A2. Map of the Northern Mountain Range region of Trinidad, with stars marking the sites used throughout this thesis (from left-right: Tunapuna, Arima, Upper Aripo, Quaré) 219 


\section{Chapter 1}

\section{General Introduction}




\subsection{Introduction}

Individual animals continually face trade-offs between major selection pressures, including current survival through avoiding predators and the production of offspring. This dynamic reflects a balance between natural selection and sexual selection. Selection should favour those individuals who are able to trade off mortality from predation and reproduction in a manner that maximizes their lifetime reproductive success (e.g. Lima \& Dill 1990; Magnhagen 1991; Sih 1994; Lima 2009). A further ecological factor vital to organisms which must be traded off against the others is the need to forage, but this is outside the scope of the current study.

In numerous social species, individuals may join groups to reduce their ambient risk of predation and(or) increase their reproductive opportunities, among other potential fitness benefits (summarized in Krause \& Ruxton 2002; Ward \& Webster 2016). It is not always clear how predation risk and mating opportunities interact to influence social grouping decisions in individual animals. My Ph.D. research focused on the potential roles of predation risk (natural selection) and sexual selection, and their interaction, in shaping social partner choice among males in a social species, the Trinidadian guppy (Poecilia reticulata). Specifically, I examined social associations among males under different predation levels, and in both the presence and absence of females, in the field in Trinidad and in the laboratory.

I focused on the behaviour of males as they have been shown to be the sex with the greater rate of movement between groups, both in the guppy (Croft et al. 2003a) and 
in a diversity of other species (e.g. primates (Sterck et al. 1997); sperm whales (Christal \& Whitehead 2001); ungulates (Ruckstuhl \& Neuhaus 2002)). A male's choice of social partner can have many fitness-related consequences; for example, being the most conspicuous male in a group can maximise a male's reproductive success (e.g. Oh \& Badyaev 2010; Gasparini et al. 2013) but can also lead to an increased predation risk (e.g. Godin \& McDonough 2003; Johansson et al. 2004; Penry-Williams et al. 2018). Whilst the guppy has been well studied in many respects (reviewed in Houde 1997; Magurran 2005), my study is the first to my knowledge to examine and characterise social interactions among males across ambient predation levels, and to test whether the presence of females (i.e. inter-sexual competition) and chemical alarm cues (indicative of an immediate predation risk) affect male-male partner associations. This research therefore utilised both field observations and laboratory experiments to test hypotheses regarding the fission-fusion dynamics of social groups and male group choice in different environments and ecological conditions (with varying predation pressure and sex ratio) in the guppy.

Below, I briefly introduce the phenomenon of social grouping and its importance. I then consider the significance of predation and sexual selection (particularly mate choice), and the trade-off between the two, on male-male social associations in the guppy, which forms the framework of my work. Lastly, I state the specific research questions that I address in detail in the following chapters. 


\subsection{Social living}

Some species live much of their lives as solitary individuals and only interact socially for the purposes of breeding. They may even defend their territory against incursion from conspecifics to protect the resources that they need. However, many other species occur in social groups. Social or group living is ubiquitous in nature and is associated with varying fitness benefits and costs (summarised in Krause \& Ruxton 2002; Ward \& Webster 2016). Benefits can include increased individual protection from predators through various mechanisms, improved feeding opportunities, greater breeding opportunities, and enhanced assistance with raising offspring as parents can receive help from other members of the group (summarised in Krause \& Ruxton 2002; Silk 2007; Ward \& Webster 2016). Costs include increases in competition for limited resources, conspicuousness to predators (Botham \& Krause 2005; Botham et al. 2005), though this is not always the case, particularly in the case of aposematic prey (Riipi et al. 2001), and individual risk of infection from socially-transmittable diseases and parasites (Krause \& Ruxton 2002; Ward \& Webster 2016). As an example, female guppies are known to spend more times in groups than males, and as a result also show a significantly higher rate of parasite infection (Richards et al. 2010).

Social assemblages can vary significantly in their level of permanence, with some groups persisting for years with little movement of individuals between groups. This type of society is exemplified by groups of female greater spear-nosed bats (Phyllostomus hastatus) in Trinidad, where aggregations of individuals remain relatively stable over long 
periods of time (McCracken \& Bradbury 1981). Other species have much more transitory groupings, with individuals within them continuously changing (Krause \& Ruxton 2002; Silk et al. 2014). The latter are referred to as fission-fusion groups, where the act of joining a social group is known as fusion, whilst an individual leaving a group can be considered a fission event (Couzin \& Laidre 2009). Many fish species demonstrate this type of fissionfusion society, including guppies (Croft et al. 2003a,b; Kelley et al. 2011).

There are many different factors that affect an individual's decision to join or leave a social group, and the relative importance of these factors may also vary across situations (Krause \& Ruxton 2002; Ward \& Webster 2016). For example, it has been demonstrated in passerine birds (specifically the house finch, Carpodacus mexicanus) that males attempt to influence their perceived sexual attractiveness through their associations with others. Less ornamented males have greater social lability, move more between social groups and selectively join particular groups in which they experience enhanced relative sexual attractiveness to females prior to the breeding season (Oh \& Badyaev 2010). Fission-fusion societies occur in taxonomically diverse species (e.g. Kerth \& Konig 1999; Couzin \& Laidre 2009; Sueur et al. 2010; Silk et al. 2014), including humans (Aureli et al. 2008).

Individuals within assemblages may not interact with all other members of that assemblage to the same degree. They may largely associate with only a small subset of the overall group, whilst interacting much less with others. This is seen in Bechstein's bats (Myotis bechsteinii) where females gather in large colonies. The overall membership rarely alters, but within these aggregations there are a number of subgroups that 
demonstrate non-random mixing (Kerth \& Konig 1999). Individual bats are able to maintain long-term social interactions, despite the large size and dynamic nature of their colony (Kerth et al. 2011). It has also been revealed that individual African elephants (Loxodonta africana) experience long-term social interactions. Core groups of individuals remain relatively constant (with group membership predicted by genetic relatedness), but each group also undergoes regular fission-fusion interactions with others to temporarily form larger aggregations (Archie et al. 2006).

Characterising social interactions within groups is important for a number of reasons, such as enhancing our understanding of the transmission of social information (e.g. Aplin et al. 2012) and diseases within and between groups (e.g. Drewe 2010; Richards et al. 2010; Silk et al. 2017; Stroeymeyt et al. 2018), and life history traits such as individual longevity (e.g. Silk et al. 2010). In addition, understanding social interactions can be important for mitigating animal welfare concerns in managed populations (Rose and Croft 2015; Sigurjónsdottir and Haraldsson 2019).

Individuals may choose to associate with kin over non-kin (e.g. Silk 2002; Archie et al. 2006; McKinnon et al. 2006), but genetic relatedness is not the only factor that can influence choice of social associates. A recent study of nine bat species found that all species examined showed a non-random social structure, but that individual social associations could not be predicted by kinship in every case (Wilkinson et al. 2019). Likewise, it has been shown that kin selection does not apparently play a significant factor in determining shoal membership in guppies (Russell et al. 2004; Croft et al. 2012). Individuals within a population typically vary in phenotype, and this can have 
consequences for social associations. Under certain conditions, such as heightened risk of predation, it may become beneficial for individuals to group with others that resemble themselves, so as to minimise the oddity effect (the predator's preference for outstanding, or conspicuously odd, individuals) (Landeau \& Terborgh 1986; Krause \& Ruxton 2002; Ward \& Webster 2016). This will be discussed further below (section 1.3). Conversely, in some species, one sex may demonstrate a preference for mates displaying a rarer phenotype, and therefore it may benefit an individual to be the phenotypically odd member of a group (Hughes et al. 1999; Dargent et al. 2019).

\subsection{Predation}

As mentioned in section 1.2, a key benefit of social living is an associated reduction in individual risk of predation (as summarised by Krause \& Ruxton 2002; Silk 2007; Ward \& Webster 2016). There are many different mechanisms by which group membership can minimise the risk of predation to an individual. The many-eyes effect states that a greater number of individuals watching out for predators can increase the likelihood an approaching predator will be detected, as seen in quelea (Quelea quelea) when approached by a bird of prey (Lazarus 1979). The selfish herd theory states that individuals group together in order to place others between themselves and potential predators (Hamilton 1971). In a similar vein, encounter-dilution is the phenomenon that while a group of animals may be more conspicuous, an individual within that group is less likely to be targeted e.g. Turner and Pitcher 1986). Predator swamping is particularly 
relevant in species such as green turtle (Chelonia mydas) where a mass-emergence of hatchlings can mean that there are far more individual prey than predators are able to capture (Santos et al. 2016). Predator confusion is most effective when individuals within a group look alike, and therefore singling out any one individual to attack is challenging for predators (Krause \& Ruxton 2002; Ward \& Webster 2016).

One behavioural consequence of predation, which is directly related to my doctoral thesis research, is assortative shoaling and the oddity effect. Positive assortment occurs when individuals who resemble each other phenotypically group together to appear as phenotypically homogeneous as possible. The "oddity effect" refers to the phenomenon of predators preferentially targeting individuals who stand out as unusual or aberrant in some way from the majority in a group (Milinski 1977; Landeau \& Terborgh 1986; Krause \& Ruxton 2002; Ward \& Webster 2016), which in turn selects for phenotypic similarity among individual members of a group. By being part of a group of phenotypically similar conspecifics, an individual therefore reduces its chance of being the one targeted and attacked by the hunting predator (Krause \& Ruxton 2002; Ward \& Webster 2016). A theoretical study demonstrated that having odd individuals in a group diminishes the confusion benefits of group membership, and that this oddity effect is more pronounced in larger groups (Krakauer 1995).

There can, however, be an interaction between oddity and crypsis, with mixedphenotype groups forming because cryptic individuals become even safer from predators as the number of conspicuous individuals in the group increases, as seen in waterfleas Daphnia sp. (Rodgers et al. 2013). The oddity which makes an individual stand out in a 
group can relate to any observable characteristic. For example, when minnows in a school are of different body sizes, an individual of a rarer size class is more likely to be taken by a predator (Theodorakis 1989). Recent work also demonstrated that waterfleas of an unusual colour were attacked by fish predators (kerri tetra Inpaichthys kerri) more often than those of a common colour, though the study did also note that the time taken to attack was no different (Penry-Williams et al. 2018). There is much evidence for sizematched shoaling in fishes (e.g. Hoare et al. 2000a; Croft et al. 2003b, 2005), and in some cases this preference for individuals of a similar size can even exceed the preference for conspecifics (for example, Krause \& Godin 1994; Hoare et al. 2000b). In addition, there is also some evidence for assortative shoaling by body coloration (e.g. McRobert \& Bradner 1998; Rosenthal \& Ryan 2005; Blakeslee et al. 2009), and even by personality type, as seen in guppies where shoals appear to be assorted by cooperative phenotypes regardless of kinship under high-predation conditions (Brask et al. 2019).

In addition to affecting individual social association decisions, predation threats can have varying non-lethal effects on the phenotype and behaviour of individuals. High predation levels can lead to the evolution of cryptic body coloration and anti-predation behaviours, such as increased vigilance behaviour and a reduction in the conspicuousness of certain behaviours, including reproductive and feeding activities (e.g. Werner et al. 1983; Lima \& Dill 1990; Endler 1995; Lima \& Bednekoff 1999; Verdolin 2006; Lima 2009).

As the field of animal 'personalities' and behavioural syndromes has developed (Sih et al. 2004; Dingemanse \& Reale 2005; Schuett et al. 2010; Stamps \& Groothuis 2010; Conrad et al 2011; Sih \& Del Giudice 2012; Sih et al. 2012), it has become evident that 
predator-mediated changes in behaviour might not be uniform across all individuals. For instance, Jones \& Godin (2010) demonstrated that individual juvenile convict cichlids (Amatitlania siquia) who were bolder and explored new surroundings rapidly were also slower to react to a potential predator threat than more timid and less exploratory conspecifics. This means that whilst bolder and more exploratory fish may have been able to feed more effectively, they were also at an increased risk of predation than more timid and less exploratory individuals.

\subsection{Sexual selection}

Animals that reproduce sexually will often have to select a partner from a field of potential mates. In some mating systems, individuals of one sex will actively compete for access to a number of individuals of the other sex (e.g. Andersson 1994), such as the previously mentioned greater spear-nosed bats. Here, males defend groups of females to obtain exclusive matings with all females in the group (McCracken \& Bradbury 1981). In other species, individuals will select their mate from a number available within the population. These decisions are often based upon specific traits preferred by members of the opposite sex, and which may advertise the fitness of the individual possessing them. An example of this phenomenon is the collared lizard (Crotaphytus collaris), where males perform "push-up" displays to attract females (Baird 2013). In addition to behavioural courtship displays, mate choice can be based on other factors such as the size of the 
territory held, as seen in the lekking black grouse (Tetrao tetrix) where larger, fitter males will also defend the most substantial territories (Alatalo et al. 1992).

Historically, there has been a biased focus on female mate choice (Andersson 1994; Clutton-Brock \& McAuliffe 2009). Females are often labelled the "choosy" sex as they are generally considered to be the sex that makes the greatest investment in reproduction as eggs are more "expensive" to produce than sperm, and also females will often undertake significant gestation and parental care (Andersson 1994). As a result, males attempt to increase their relative sexual attractiveness to potential mates. This can include selecting which male conspecifics they associate with to appear relatively more attractive to potential mates (as with the previously mentioned house finches, Oh \& Badyaev 2010). There has been a recent movement away from this focus on solely female choice, as it is increasingly acknowledged that males can also be choosy and both sexes incur costs during mate selection (Bonduriansky 2001; Guevara-Fiore et al. 2010; and reviewed in Edward \& Chapman 2011; Schlupp 2018). In terms of assessing potential mates, there are advantages to group living for both sexes, as individuals can observe the mating interactions of others and obtain social information about the relative suitability of different individuals as potential mates. This can be intentional, in terms of courtship displays, or inadvertent through observing the decisions of others (Danchin et al. 2004).

Observation of mating decisions can lead to mate-choice copying. This has been demonstrated in a number of species, where individuals given a choice of potential mates will select one which they previously observed being preferred by another partner (e.g. Dugatkin \& Godin 1992; Briggs et al. 1996; Witte \& Ryan 2002; Ophir \& Galef Jr 2003; 
Witte \& Massmann 2003; Alonzo 2008; Galef Jr et al. 2008). Alternatively, witnessing a previous copulation event can also decrease a potential mate's preference; for example, one study showed that female fruitflies (Drosophila melanogaster) would avoid a male they had seen mating recently, as they were perceived to be sperm-depleted and would not be able to fertilise all of the female's eggs (Loyau et al. 2012). There is also evidence that the perceived level of competition for mates between males can affect a focal male's courtship behaviour, as the presence of a bystander can lead a male to court a female that would not normally be their preferred choice, in order to reduce the risk of remaining unpaired (Dubois \& Belzile 2012).

\subsection{Trade-offs}

As mentioned in Section 1.1, both sexual selection and predation are important evolutionary selection agents that can shape animal behaviour. It is important to note that these agents are often in conflict with one another. This is demonstrated by the wolf spider Hygrolycosa rubrofasciata, where males signal for females by drumming their

abdomens, but while males exhibiting high drumming rates are favoured as mates by females, these males are also at greater risk of predation (Kotiaho et al. 1998). It is clear that there is a trade-off at work between sexual selection and predation, which has been discussed in several review articles (e.g. Lima \& Dill 1990; Magnhagen 1991).

When examining this trade-off, it is important to note that there may also be other factors in play. Further examination of $H$. rubrofasciata found that males with higher 
signalling rates also appeared to be more adept at escaping from predators (Lindström et al. 2006). A study of sailfin mollies (Poecilia latipinna) found that generally females in high predation-risk habitats produce a large number of small offspring, and those in low predation-risk habitats produce fewer, larger offspring. However, this trend was not universal across the study system's range, and the authors hypothesise that additional factors, such as food availability, may also have an effect on the female's reproductive strategy (Krebs \& Bell 2012).

\subsection{Thesis objectives and rationales}

Previous research on social grouping has focused a great deal on the decisions made by females regarding whom to associate with. In many species demonstrating fission-fusion dynamics, it is the females that spend more time in groups, whilst males frequently move between groups in search of sexually-receptive mates and thus have shorter group tenures (Bisazza \& Marin 1995; Sterck et al. 1997; Christal \& Whitehead 2001; Ruckstuhl \& Neuhaus 2002; Croft et al. 2003a). As a result, many of the current studies on the dynamics of social grouping and choice of associates focus on females. When considering social associations in a reproductive context, there has been much work on how individuals select potential mates, but much less on their interactions with others of the same sex during the search for these mates. Where male-male social interactions have been considered (e.g. Oh \& Badyaev 2010; Auge et al. 2016), there is a paucity of information on how predation risk could affect such social decisions. This 
should be considered because individuals should be selected to seek social environments in which both their survival and reproductive success are enhanced as much as possible (Lima \& Dill 1990; Krause \& Ruxton 2002; Ward \& Webster 2016). The increased risk of predation may make non-random preferences for conspicuous shoal mates less common because such sexually attractive males are also more commonly targeted by predators (Endler 1980; Godin \& McDonough 2003).

My thesis research focuses mainly on male-male social associations and investigates whether males associate non-randomly with other males, and whether they demonstrate a preference for a specific phenotype in male social partners. For example, a more ornamented male is likely to be more sexually attractive to females (Magurran 2005; Auld et al. 2016), and therefore may be a less favourable shoal mate for a drab male who appears less attractive by comparison (Bateson \& Healy 2005; Gasparini et al. 2013). Alternatively, having more attractive shoal mates could increase a focal male's exposure to receptive females as they are attracted by the presence of such shoal mates.

The overarching aim of my doctoral work is to quantitatively characterise the social environment of Trinidadian guppies in different populations that have evolved under different levels of predation intensity and to investigate social interactions among males (i.e. choice of social partners) under different ecological and social contexts. Phenotypic preference is considered using body length and body coloration, two traits that are under both natural and sexual selection in numerous species, including my model study species, the Trinidadian guppy (e.g. Andersson 1994; Lima \& Dill 1990; Houde 1997; Auld et al. 2016). In particular, I examine whether and how ambient predation level 
affects group-choice decisions in males, as males strive to remain alive whilst maximising their mating opportunities and future fitness (Lima \& Dill 1990). Whilst there has been consideration of the trade-off between anti-predator behaviour and reproduction (Magnhagen 1991), the role of social groupings and social interaction decisions have so far received little attention. In this thesis, I examine the interplay between natural and sexual selection and their effects on the social environment choice of male Trinidadian guppy. The guppy is a well-established model study species in ecology and evolution (Houde 1997; Magurran 2005), and I hope that my findings will be applicable to other species with similar social dynamics, particularly as the majority of species will have to address the trade-off between predation risk and reproductive output. The suitability of the guppy as a model for my research study is discussed further in Chapter 2 (General Methods).

The main questions addressed in my thesis are as follows:

How does population of origin affect male decisions to join and leave social groups and their behaviour within groups?

This question is addressed in Chapter 3 with observations of shoaling dynamics in a number of rivers in Trinidad that differ in the number and density of fish predators (amongst other factors, Endler 1995; Magurran 2005). I quantified the rate of joining and leaving shoals (fission-fusion dynamics) and shoal tenure duration by free-ranging focal males. The reproductive behaviour of focal males was also quantified through the frequencies of sigmoidal courtship displays and sneak copulation attempts (cf. Houde 
1997). I predicted that males in low predation-risk populations would be more mobile and spend less time as a member of a shoal, whilst entering more shoals in search of mates, owing to the lower risk of predation associated with being outside of a shoal (Luyten \& Liley 1985; Kelley et al. 2011), compared to males in high-risk populations. I also predicted that males in low-predation populations would exhibit higher rates of sigmoidal courtship displays and lower rates of sneak copulation attempts (Luyten \& Liley 1985), as courtship displays are more likely to result in a successful insemination (Pilastro \& Bisazza 1999) and the risks to males in these low-predation sites are lower than in high-risk populations. Conversely, I predicted that males in high-predation sites would tend to remain in shoals longer, move between shoals less frequently and spend less time alone, due to the risk of predation that such behaviour would entail (Seghers 1974; Magurran \& Seghers 1991; Kelley et al. 2011). I predicted that shoals would tend to be larger in high-risk sites, so as to dilute individual risk of predation, than in the low-predation sites. I predicted that males in high-predation sites would also perform a greater level of sneak copulation attempts with fewer sigmoidal courtship displays, as the conspicuous nature of courtship displays would cause them to be potentially more costly in high-risk sites than low-risk sites (Luyten \& Liley 1985; Magurran \& Seghers 1990; Godin 1995).

Do males preferentially associate with other males of a specific phenotype (more or less attractive than themselves), and how does population of origin and female presence affect such decisions? 
This question was addressed in Chapter 4 using wild-caught fish in a laboratory setting. Focal males were offered a dichotomous choice between shoals containing more and less sexually attractive (in terms of both total body size and coloration) stimulus males, either with or without a female in the stimulus shoals, and time spent in the vicinity of each shoal was recorded as a proxy for shoaling. I predicted that males would prefer to shoal with the group containing the male(s) less attractive than themselves so as to maximise their own relative sexual attractiveness (Bateson \& Healy 2005; Gasparini et al. 2013), and that this preference would be stronger when females are present due to sexual competition (similar to findings in Oh \& Badyaev 2010) and strongest in males from low predation-risk populations of origin because these males would experience less risk by being the outstanding (phenotypically odd) individual in a group. I predicted a weaker preference in focal males from high-risk populations as they might encounter greater risk as a result of being the conspicuous individual in a shoal (Endler 1980; Godin \& McDonough 2003; Johannson et al. 2004).

\section{Do females change their preference for a specific male phenotype based on the number} of males present, and does population of origin affect this?

Given the findings of Chapter 4 (where focal males were found to prefer shoals of more attractive males in the absence of females), this question was addressed in Chapter 5 using wild-caught fish in a laboratory setting to test whether female preference for a specific male phenotype was altered by the number of males present displaying that 
phenotype. Focal females were presented first with a choice of two stimulus males differing in their sexual attractiveness based on body coloration. After a first preference test, females were either presented with the same two males again for a second preference test, or each of the males were presented as part of a shoal of three males, where all males in a shoal were closely matched for overall body coloration. My prediction was that, when tested with the two single males twice, females would show a preference for the more colourful male in the first preference test (Houde 1997; Auld et al. 2016), and then this preference would increase in the second preference test (as the female had already established her preference, meaning further sampling should not alter this). When two shoals of 3 males are presented in the second preference test, I hypothesised two potential outcomes. Either the female would show an even stronger preference for the more attractive shoal after initial sampling, as it contained multiple males who would make high-quality mates. Alternatively, the females from the high-risk populations in particular would show preference for the less attractive shoal, as these males were less conspicuous and therefore this group would be less likely to draw the attention of potential predators. Female guppies are much more cryptically coloured than males, and studies in both guppies and other species have shown females can incur increased predation costs by associating with visually conspicuous males (Pocklington \& Dill 1995; Segami Marzel et al. 2017). 
Does the presence of a chemical alarm cue alter the phenotypic shoaling preferences of focal males in favour of the shoal most similar to their own phenotype, and does population of origin affect this?

As Chapters 3, 4 and 5 considered the effect of ambient population level predation risk without exposing individual guppies to any immediate threat of predation, Chapter 6 examined whether the presence of a chemical alarm cue, indicative of an immediate predation risk (Brown and Godin 1999), would alter the shoaling preferences of focal males. In this experiment, I offered focal males a choice of two shoals, one wherein conspecific males were phenotypically similar to the focal male, and one wherein the males were phenotypically similar to each other, but distinct from the focal male. Some focal males were exposed to a chemical alarm cue prior to making this choice; others received a water control. Based on the findings of Chapter 4, I predicted that, in the absence of chemical alarm cues, males from both populations would prefer to shoal with the group of males who were phenotypically most sexually attractive (largest and with more body coloration), regardless of the focal male's personal phenotype. However, I also predicted that, when tested in the presence of the alarm cue, focal males from the highrisk population should prefer to shoal with the other males most phenotypically similar to themselves (i.e. a less attractive focal male will prefer the shoal of less attractive males, whilst a more attractive focal male should prefer to associate with the more attractive males), presumably to minimise the oddity effect and the risk of being a phenotypically distinct individual within a group (Milinski 1977; Landeau \& Terborgh 1986; Krause \& Ruxton 2002; Ward \& Webster 2016). Previous work with female guppies has 
demonstrated size-assortative shoaling in high-risk situations (Hasenjager \& Dugatkin 2017). 
Chapter 2

General Methods 


\subsection{Study Species}

The Trinidadian guppy is a highly-social, live-bearing and sexually dimorphic freshwater fish native to Trinidad and nearby islands (Magurran 2005). Females are larger than males and are a uniform olive colour, whereas the smaller males exhibit diverse body colour patterns, consisting of orange/yellow/red (carotenoids/pterydines), black (melanin) and iridescent patches (Houde 1997; Magurran 2005). Male body coloration and body length varies greatly among males both within (see Appendix Figure A1 for an example) and between populations, meaning there is an extreme diversity of phenotypes (Endler 1980, 1983; Houde 1997; Magurran 2005; Auge et al. 2016). Males will not increase in body length significantly once sexual maturity is reached, but females are able to continue growing past this point (Magurran 2005). It appears that more brightly coloured males are at a greater risk of predation from predatory fishes (Endler 1995; Godin \& McDonough 2003). Throughout my thesis, where male colour or ornamentation are mentioned, I refer to the proportion of a male's body covered with orange and black pigmentation.

Male guppies can reach sexual maturity after as little as 7 weeks post-birth, whilst female guppies tend to mature between 10 and 20 weeks and may reproduce until 20-34 months old (Magurran 2005). Both sexes exhibit mate choice, with females tending to prefer larger, more ornamented males (Houde 1997; Magurran 2005; Auld et al. 2016), though preference for rare phenotypes has also been demonstrated (Hughes et al. 1999; Dargent et al. 2019). Males generally prefer larger, and therefore more fecund, females 
(Herdman et al. 2004; Jeswiet et al. 2012; Godin \& Auld 2013). In the Quaré River population, this preference is apparently only demonstrated when males can accurately assess the mating status of females (Herdman et al. 2004). As with females, there is also evidence that males have a preference for unfamiliar mates (Kelley et al. 1999).

Male guppies can utilise two different tactics to achieve matings. One tactic is the courtship display, where a male presents himself to a female, bending his body into a sigmoidal shape and vibrating it vertically (Houde 1997). Alternatively, males can attempt a sneak copulation, whereby a male approaches a female from behind and attempts to insert his gonopodium and inseminate her without courtship, also known as a gonopodial thrust or coercive mating attempt (Liley 1966; Houde 1997; Matthews \& Magurran 2000). Although both tactics can result in insemination, courtship is generally more successful and results in a higher level of sperm transfer than sneak copulation (Pilastro \& Bisazza 1999). Female guppies are only sexually receptive when they first reach maturity and for a brief period (2-4 days) following parturition or birth of a brood, but they are able to store sperm for later use (Houde 1997) and their broods are normally multiply sired by different males (Kelly et al. 1999; Neff et al. 2008). The sneak copulation strategy allows males a chance to inseminate even these non-receptive females.

Most previous studies of guppy social behaviour have focused on the social decisions of females, as females spend more time in shoals than males and are generally considered the more social sex (Magurran 2005). There is evidence that free-ranging shoals of guppies (largely comprised of females) can be assorted by size (Croft et al. $2003 b, 2005,2009 a)$, and also that females will prefer to shoal with larger females when 
offered the choice (Jones et al. 2010). It has been suggested that size-assorted shoaling in wild guppies may be partly due to the difference in ability to utilise different habitats due to the size of individuals (i.e. environmental constraints), rather than individual preferences for specific companions (Croft et al. 2003b, 2004a).

As a result of their more transient nature (regularly moving between shoals in search of mating opportunities; Croft et al. 2003a), less consideration has been given to the social associations of males. It is clear, however, that males also have decisions to make when choosing other males to associate with. While larger, more ornamented males are likely to be more attractive potential mates to females (Magurran 2005; Auld et al. 2016), smaller, less ornamented males may avoid prolonged associations with such attractive males to avoid appearing less desirable by comparison (Bateson and Healy 2005; Gasparini et al. 2013). Alternatively, it may be advantageous for males to associate with more colourful males, as whilst such males are more appealing to females they can also only court one female at a time, and therefore the female's shoal mates may be available for the less attractive male to pursue. A previous study did not find any evidence for social partner preferences, and assortative shoaling, based on body coloration or body size in male Trinidadian guppies, with males apparently socially associating with each other randomly (Auge et al. 2016). The latter study only considered individuals from a single natural Trinidadian population. My thesis aims to expand on the latter findings by comparing social partner choice in male guppies originating from different riverine populations in Trinidad and therefore subject to different levels of predation to consider 
the potential effects of multiple factors (i.e. predation risk as well as reproductive drive) on male-male social associations.

Natural populations of guppies have evolved under different predation regimes, as demonstrated by a number of studies which have quantified the type and number of predators present in different rivers and in different sections of the same river (e.g. Endler 1995; Croft et al. 2006b; Botham et al. 2008). As an example, the Upper and Lower Aripo Rivers have different predation environments as waterfalls provide physical barriers preventing fish predators from colonising areas further upstream (Houde 1997; Magurran 2005). Predation levels were previously established based solely on the presence or absence of a major piscivorous predator, the pike cichlid (Crenicichla frenata), but recent studies have considered a broader spectrum of potential predator species, including blue acara cichlids (Aequidens pulcher), wolf fish (Hoplias malabaricus) and killifish (Anablepsoides hartii) in addition to the pike cichlid, which represents a more comprehensive range of threats to the guppy, and in situ measures of their respective attack rates on guppies (Croft et al. 2006b; Botham et al. 2008). There have been studies which classify predation in different ways (i.e. using total predator biomass, presence/absence of specific predators, or by attack rate), and these can sometimes contradict one another in part. Whilst predator biomass can be a valid measure, it is important to also consider the diversity of predatory species present in the river. A recent study (Deacon et al. 2018) quantified predation level as the biomass of predators present, meaning some traditionally low-predation rivers were reclassified as high predation due to the abundance of the killifish A. hartii. A. hartii is a small, gape-limited predator of 
juvenile guppies only, and therefore does not pose a significant threat to adult guppies. As my work focuses solely on the behaviour and threats to adult guppies, I will classify rivers based on the presence/absence and attack rate of fish predators of adult guppies only. It should be noted that other taxa such as spiders may present a predation risk to guppies (Deacon et al. 2015), but this has not been quantified.

My work utilises fish from four genetically-distinct riverine populations (see Appendix Figure A2 for map). Throughout my thesis, I use guppies from a high-predation river (the Quaré River) and a low-predation river (the Tunapuna River). In addition, in Chapters 3 and 4, I use an intermediate-predation risk population (the Arima River), and in Chapter 3 also use a second low-predation risk population (the Upper Aripo River). These rivers have been classified for predation risk through personal observations and following the categorisation of Croft et al. (2006b), based on the presence of fish predator species (cichlid sp., wolf-fish and killifish) only. It should be acknowledged that these sites will vary in other conditions (including food availability and light levels), and so whilst the studies which follow can be considered a comparison between populations, it should not be believed that any differences observed are solely the result of the different predation risks experienced in the riverine populations.

It has previously been shown that guppies from high-predation risk populations exhibit different behaviours from their low-predation risk conspecifics (reviewed in Endler 1995; Magurran 2005). In particular, a reduction in the flamboyant courtship display is seen, with an increased level of less conspicuous sneak copulations (Endler 1987; Godin 1995). Guppies from high-predation populations also show different phenotypic 
characteristics, as they are generally smaller and have duller male body coloration on average (as ostentatious colours evolved to attract the attention of a mate will also be more easily detected by and attractive to potential predators) than those from lowpredation populations (Endler 1980; Godin \& McDonough 2003). In addition, there can be sex segregation by habitat in high-predation areas as conspicuous males tend to prefer shallow waters where they are somewhat protected from predators, whereas females are found in deeper waters where they can minimize their risk of male sexual harassment (Croft et al. 2006b). Males in high-predation populations are also known to reach sexual maturity at a smaller size than conspecifics from low-predation populations, allowing them to begin reproduction earlier (Reznick and Edler 1982).

It has been demonstrated in the laboratory that male guppies preferentially associate with male conspecifics that are less attractive to females than themselves and thereby presumably increase their own relative sexual attractiveness and chances of mating successfully (Gasparini et al. 2013, but see Auge et al. 2016). However, there has not yet been consideration of whether this type of social association will be affected by ambient predation level. Since more visually attractive males are also more conspicuous to predators (Endler 1980; Godin \& McDonough 2003), such non-random social associations may be less likely to occur in high-predation populations than low-predation ones. 


\subsection{Fish Care in the Laboratory}

All guppies utilised in my doctoral thesis-related research were captured using random seining from each of the study rivers mentioned above, and were transported in buckets of river water by car to a laboratory at the University of West Indies, St. Augustine campus. They were held in large, mixed-sex aquaria containing aged tap water $\left(24-26^{\circ} \mathrm{C}\right)$ under a $12 \mathrm{~h} \mathrm{~L}: 12 \mathrm{~h}$ D illumination regime and fed twice daily with commercial flake food until used in behavioural trials. When wild-caught fish first arrived at the laboratory, they were treated with Fungus Guard, Pimafix and Melafix (following manufacturers' instructions) to prevent fungal, bacterial and parasitic infections ensuring only healthy fish were used in my experiments. For the majority of experiments, I maintained the guppies in a mixed-sex environment prior to testing which ensured that the vast majority of females were gravid and therefore not sexually receptive. This mimics the situation in the wild (Houde 1997) and means that it is unlikely that female sexual behaviour influenced the social partner choices made by focal males during behavioural trials.

\subsection{Dichotomous Choice Tests}

The dichotomous choice paradigm is commonly used in a range of behavioural choice studies in animals, and particularly in guppy studies (summarised in Houde 1997). In such a choice test, a focal male guppy is placed in a central test aquarium and presented simultaneously with two stimulus shoals located in containers placed adjacent to either end of the central aquarium (Figure 2.1). A 10-cm wide (approximately 4 body lengths) 
area at either end of the central aquarium is designated as the social association zone for the adjacent shoal. Social association time (i.e. total time spent in a given association zone) is considered a reliable proxy of shoaling preference, as confirmed in freeswimming trials for the guppy (Jeswiet \& Godin 2011). The external sides of all tanks were covered in brown paper to minimise potential external visual disturbances to the guppies during behavioural trials. Focal and stimulus fish were always selected from different stock tanks to minimise any potential preference effect caused by individual familiarity, which can develop after 12 days of social association in the guppy (Griffiths \& Magurran 1997; Croft et al. 2004c). 


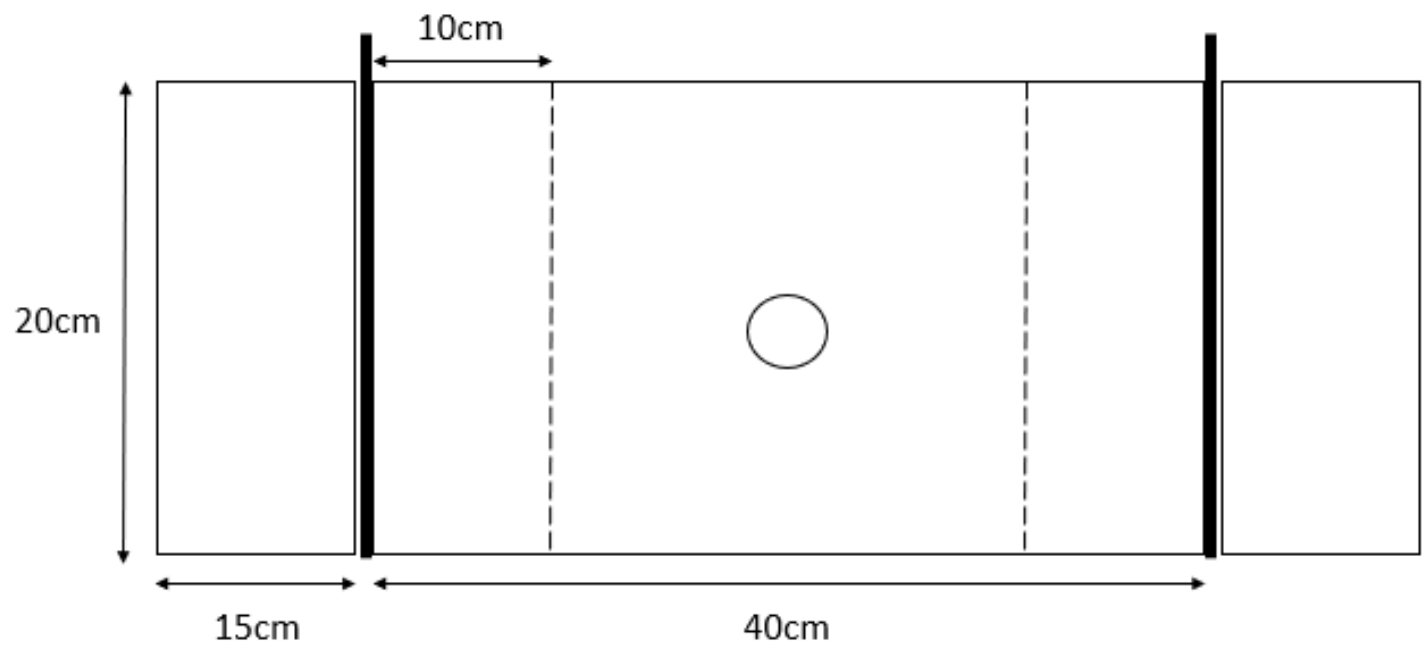

Figure 2.1 Top view of a schematic of the experimental apparatus, with measurements. Dotted lines indicate the boundary of the $10-\mathrm{cm}$ preference zone (marked beneath the tank using marker pen on Styrofoam) adjacent to each of the stimulus fish end compartments. The circle in the central test aquarium denotes a clear Plexiglas cylinder (7 cm diam.) that temporarily held the focal male guppy during the acclimatisation period prior to choice tests. Thick black lines indicate removable opaque Plexiglas screens that initially blocked the view of the focal male into the end stimulus fish compartments. The central test aquarium and end compartments were constructed of clear Plexiglas. 
Though Figure 2.1 demonstrates a typical experimental apparatus, the exact setup and recording of movement in choice tests varied between my different experiments and are stated in the Methods sections of each of the relevant chapters (Chapters 4-6) which follow. In brief, a focal male was initially placed in a clear Plexiglas cylinder $(7 \mathrm{~cm}$ diam.) in the centre of the test aquarium and the two stimulus shoals in the end compartments were obscured from view by opaque Plexiglas screens. Both the focal and stimulus fish were given a period of time to acclimatise to the apparatus without seeing one another. After this acclimatisation period, the screens were removed, and the fish were again left to acclimatise further whilst in view of each other. The precise duration for the acclimation period varied between experiments and is stated in each chapter. The central cylinder was then gently lifted and the focal male allowed to free-swim in the central aquarium and to choose to associate with either stimulus shoals for $10 \mathrm{~min}$. The movement of the focal male was video filmed from above using an HD webcam (Logitech C920 HD Pro) mounted $40 \mathrm{~cm}$ above the aquarium and coupled to a laptop computer, which allowed the observer to record movement without disturbing the fish in the apparatus. Any focal male that did not enter both preference zones during the first 10min preference trial was removed from further testing and analysis.

Following this first 10 -min preference test, the focal male was gently returned to the central cylinder and the opaque screens were replaced, blocking the stimulus shoals from view. The positions of the compartments containing the stimulus shoals were then switched to control for any potential side bias of the focal fish. Following a second acclimatisation period, the preference test was repeated for another $10 \mathrm{~min}$. The time 
the focal fish spent in either association zone was summed over the two 10-min preference tests as a measure of its association (shoaling) preference for either stimulus shoal. Any fish that spent more than $80 \%$ of its total shoaling time (i.e. the time that it was in one or other of the two association zones) on one side of the tank across the two preference tests was considered to have exhibited a side bias (criteria first established in Schlupp et al. 1994) and was therefore discounted from further analysis. Appendix 1 confirms that this procedure had no confounding effect upon the subsequent analysis.

\subsection{Measurements of Fish Body Length and Coloration}

When guppies were initially selected for use in a behavioural trial, their total body length was measured approximately using a Perspex ruler and male coloration was assessed by eye. At the end of the trial, all fish were lightly anaesthetised in turn using MS-222, individually placed on a piece of white Plexiglas and their left side photographed using a Nikon Coolpix P7800 digital camera alongside a metric ruler. Once revived, the fish were then placed in holding aquaria. At a later date, the body length and coloration of individual fish were more accurately quantified from their digital photograph using ImageJ software (Schneider et al. 2012). The total body length (tip of snout to end of caudal fin), total area of the left side (excluding tail and fins) and the areas of the left side of the body covered in orange and black pigmentation (excluding tail and fins) of individual males were quantified. Other coloration (such as iridescence or UV areas) were not measured as the camera was not able to accurately capture them. Measurements 
were taken using a Wacom Intuos Creative Pen Tablet in order to maximise accuracy.

Statistical analysis of the data was then performed using R (v3.5.2., R Core Team 2018). 


\section{Chapter 3}

Sex, lives, and videotape:

A cross-population comparison of the social and sexual behaviours of free-ranging male Trinidadian guppies 


\subsection{Abstract}

Predation is an ecological selective agent that can have significant effects on the appearance and behaviour of individuals in a population over both ecological and evolutionary time scales. Here, I quantified and compared the phenotypes and both the social and sexual behaviours of free-ranging male guppies within three natural Trinidadian populations (four for phenotypic comparison) that have evolved under differing fish predation levels. I used seine net surveys to quantify the density and adult sex ratio of the guppy populations and photographed collected males individually to determine their body length and overall level of body coloration. I also video recorded randomly-selected individual ('focal') males in their natural habitats and used these videos to quantify their social and sexual behaviours. I found no significant differences in guppy density or sex ratio among populations but did observe some phenotypic differences. The largest males were found in the low-predation Upper Aripo River population. The males in the intermediate-predation Arima River population were the least colourful, though this may be more due to the effects of high turbidity levels (owing to local mining activity) at the site sampled in this river than anything linked to predation. There was no significant difference in social behaviours among the populations. However, there were some differences in sexual behaviour, most notably the higher rate of male sneak copulation attempts in the high-predation Quaré River population compared with the other two populations. This work demonstrates that male guppies from different populations do differ in their mean physical and behavioural phenotypes, which may partly be explained by the different predation regimes they experience in nature. 


\subsection{Introduction}

Predation is a driving force of natural selection within animal populations (Lima \& Dill 1990; Magnhagen 1991; Krebs \& Davies 1993; Relyea 2003; Ruxton et al. 2018). Prey species can use many different strategies to avoid predation. They may evolve cryptic or background-matching traits and toxic defences (reviewed in Ruxton 2009; Stevens \& Merilaita 2009; Ruxton et al. 2018). There can also be induced morphological defences that occur only in the presence of predators. For example, tadpoles of the gray treefrog (Hyla chrysoscelis) exhibit morphological changes induced by the presence of predatory dragonfly larvae. When these larvae are present, the tadpoles develop a shorter, deeper tail fin, which appears to aid predator avoidance (McCollum \& Leimberger 1997). Morphological change in response to predation cues can also be seen in the goldfish (Carassius auratus), where individuals exposed to chemical predation cues grow deeper bodies and gain more weight, and later show better survival when exposed to predators, compared with shallow-bodied conspecifics (Chivers et al. 2008). Life history traits can also vary in closely related species according to the predation environment they experience. For example, some cladoceran species will delay reproduction until they reach a size too large to be significantly at risk of predation, whilst smaller species begin to reproduce earlier as they will never outgrow their predation risk and should therefore focus on reproducing as soon as possible (Lynch 1980). A similar trade-off is observed in populations of guppies that experience different predator communities. Guppies that have evolved under high fish predation levels begin reproduction at a smaller size, and 
have shorter intervals between broods, than guppies in low-predation risk populations (Reznick \& Endler 1982; Reznick et al. 2001).

In addition to these physical differences, animals may exhibit context-dependent behavioural responses to predation risk (summarised in Lima \& Dill 1990; Sih 1994; Verdolin 2006; Lima 2009). One such example is seen in the orange-crowned warbler (Vermivora celata) which exhibits a plastic nesting strategy, shifting to more concealed ground nest sites upon the introduction of an avian predator (Peluc et al. 2008). Female song sparrows (Melospiza melodia) produce significantly fewer offspring when they perceive an increased predation risk (Zanette et al. 2011). Similarly, oldfield mice (Peromyscus polionotus) favour foraging sites under cover, rather than those which are exposed, as these provide a reduced risk of predation (Orrock et al. 2004).

Increased predation can also promote group living, which reduces individual risk of predation (summarised in Krause \& Ruxton 2002; Ward \& Webster 2016, and discussed in more detail in Chapter 1). Group living can be beneficial in the presence of predators due to the increased collective vigilance provided by many eyes (Lima 1995), as well as the risk dilution and predator confusion effects that being part of a group can provide (Krakauer 1995; loannou et al. 2008). Predation can also alter the dynamics of fissionfusion societies (introduced in Chapter 1, section 1.2), reducing the rate at which individuals move between groups (Kelley et al. 2011).

Separate populations of the same species can encounter different levels of predation risk and in response may evolve different anti-predator morphological, 
chemical and (or) behavioural traits. For example, in the bison (Bison bison), optimal group size can vary depending on the habitat being occupied, as different habitat types present different levels of predation risk (Fortin et al. 2009). A comparison of populations of the oriental fire-bellied toad (Bombina orientalis) found that individuals from the Jeju Island population had less brightly coloured ventral sides, as well as reduced activity levels and a preference to rest underwater rather than on rocks, in contrast to populations from the South Korean mainland. It was also found that the Jeju population was subject to a higher predation level than the mainland populations, which may well explain the difference in both behaviour and morphological phenotype between these two populations (Kang et al. 2017). An extensive body of evidence shows that different riverine populations of the Trinidadian guppy experience different fish predator communities and, as a result, have evolved population-specific life histories, morphology and behaviour (summarised in Endler 1995; Magurran 2005, and discussed in more detail below).

The guppy is a well-studied model species (summarised in Houde 1997; Magurran 2005) in evolutionary and behavioural ecology, and is endemic to rivers, streams and swamps in Trinidad. The populations in the different rivers are geographically isolated from one another, and even populations within the same river can be considered largely distinct due to the presence of barrier waterfalls, which generally prevent individuals from moving upstream (Magurran 2005). Each of these populations experience a variety of different ecological conditions (Endler 1995), including a difference in the fish predator community that they encounter. Predation levels have been quantified in several studies, 
particularly by Croft et al. (2006b) who quantified predator attack rates targeted towards a captive guppy in an in-situ submerged bottle to estimate population-level predation intensity (though other methods of classification have also been used such as total predator biomass (Deacon et al. 2018), as discussed in Chapter 2, Section 2.1). There is an extensive body of work which considers the effects of predation on guppy populations, life history, morphology and behaviour. These effects are varied, including phenotypic characteristics, shoaling behaviour and foraging activity (summarised in Endler 1995; Magurran 2005). In brief, guppies from high-predation risk populations tend to be smaller and less colourful (Endler 1980), tend to shoal more (Seghers 1974; Magurran \& Seghers 1991; Botham et al. 2008) and show reduced nighttime foraging activity in populations where nocturnal predators are present (Fraser et al 2004). Male guppies in high-risk populations have also been shown to alter their habitat use and favour shallower areas, where the risk of predation from larger fish is reduced (Croft et al. 2004a).

As noted in Chapter 2 (Section 2.1), guppies are sexually dimorphic, both phenotypically and in terms of their shoaling behaviours. Much of the existing literature on guppy sociality focuses on females as they are the sex that spends more time shoaling (Magurran 2005), and even exhibit a preference for stable social partners, indicative of long-term familiarity (Griffiths \& Magurran 1998; Croft et al. 2006a). Males are transitory and move between groups more in search of potential mates (Griffiths \& Magurran 1998; Croft et al. 2003a, b; Magurran 2005). It appears such movements may be further advantageous to males as female guppies show a preference for novel males as mates (Hughes et al. 1999). There is evidence that the degree of male sociality varies with 
population predation risk, with males from high-predation risk populations having social networks where they interacted with fewer other males than individuals in low-predation risk populations (Edenbrow et al. 2011).

In the current study, I quantified and compared the social and sexual behaviours of wild, free-ranging male guppies in three riverine populations in Trinidad that differ in their ambient predation risk (amongst other factors, Endler 1995). This was done in two ways. First, I used seine nets to take random samples of guppies from each of these rivers to estimate local fish density and adult sex ratio in the sampled areas, and to collect male guppies to photograph for body length and coloration measurements. Second, I followed randomly-selected focal individual males with video cameras and subsequently quantified their social and sexual behaviours using video playback. The social behaviours quantified were the percentage of total observation time spent shoaling, the rate of shoal encounters, shoal tenure duration and the maximum number of adult guppies within the shoal occupied by the focal individual. These metrics allowed me to assess how much time focal males were spending alone and in shoals and how frequently they moved between shoals. As noted earlier (Chapter 2, section 2.1), male guppies can use two different mating tactics: a conspicuous courtship display tactic or a sneak copulation tactic (Liley 1966; Houde 1997; Matthews \& Magurran 2000). Males have been shown to reduce the frequency of conspicuous courtship displays in favour of sneak copulation attempts in the presence of a perceived increase in local predation risk (Magurran \& Seghers 1990; Godin 1995). I recorded all instances of both courtship displays and sneak copulation attempts exhibited by individual focal males, as well as all instances of male-male competitive 
behaviour. Apparent male-male sexual competition occurs in the form of courtship interference, where a male guppy will place himself between a courting male and a female, thus interrupting the latter's courtship attempt (Houde 1997; Kelly \& Godin 2001). This type of competition does not occur frequently (relative to overall courtship and sneak copulation behaviours) and is even less common in the presence of predators (Kelly \& Godin 2001).

I set out to sample guppies from four different rivers wherein they experience differing levels of predation, namely, the Upper Aripo River (low-predation), the Tunapuna River (low-predation), the Arima River (intermediate-predation), and the Quaré River (high-predation), as discussed in Chapter 2, section 2.1. These predation level categorisations are based partly upon previous studies (e.g. Croft et al. 2006b; Botham et al. 2008), but also on my personal visual observations of fish predator abundances at the start of this work, as ambient levels of predation in rivers can vary significantly between sites. It should be noted that these populations also differ in other ecological conditions (for example, light intensity or substrate type) as well as predation intensity, and so any observed differences in guppy density, sex ratio, behaviour and morphology reported in my thesis may not be solely (or even largely) the result of population differences in predation regimes (Endler 1995). The density and sex ratios of guppies in a population may also have an impact on the behaviour of focal males; for example, higher guppy densities may lead to increased sociality, a more female-biased sex ratio may lead to higher rates of courtship behaviour (Jirotkul 1999), or a more male-biased sex ratio may lead to increased sneak copulation attempts (Řežucha \& Reichard 2014; Chuard et al. 
2016). If density and sex ratio vary significantly between populations, then they should be considered as demographic factors that potentially could influence guppy social and sexual behaviours.

As seen in previous studies of guppies (for example Endler 1980), I expected that male guppies from low-predation populations (the Upper Aripo and Tunapuna Rivers) would be on average larger and more colourful (more orange and black pigmentation on their bodies) than their conspecifics in a high-predation risk population (the Quaré River).

I predicted that males from a high-predation riverine population (the Quaré River) would spend more time within a shoal (Seghers 1974; Magurran \& Seghers 1991), and that overall average shoal membership size would be larger. I expected that, on average, males from high-predation populations would perform fewer conspicuous sigmoidal courtship displays and more sneak copulation attempts, so as to minimise their visibility to predators (Luyten \& Liley 1985; Magurran \& Seghers 1990; Godin 1995), compared with males from low-predation populations. It has previously been shown that male guppies perform fewer courtships and increased sneak copulation attempts in the presence of a greater number of rival males (Auld et al. 2015). Therefore, if I expect larger shoals in high-predation populations, then I should expect to see an increase in males attempting sneak copulation attempts over courtship displays also. In contrast, I expected that males from the low-predation populations (Upper Aripo and Tunapuna Rivers) would have shorter shoal tenures, as there is presumably less risk involved in moving between shoals, and I predicted that guppies in these two populations would generally be found in smaller groups (following the reduced shoaling tendencies observed in low-predation 
populations by Luyten \& Liley (1985); Kelley et al. 2011) than those in higher-risk populations. I also expected that Upper Aripo and Tunapuna males would exhibit higher rates of conspicuous sigmoidal courtship displays (Luyten \& Liley 1985) over the alternative sneak copulation tactic, as they should be less vulnerable to predation whilst courting females and courtship displays tend to result in a greater level of sperm transfer (Pilastro \& Bisazza 1999). In addition, I predicted that male-male courtship interference would be seen more frequently in the low-predation populations (as per Kelly \& Godin 2001).

\subsection{Methods}

\subsubsection{Seine sampling}

Data were collected during April and May 2015, with the exception of the phenotypic data for the Upper Aripo fish which were collected in April-June 2013 (Auge et al., 2016). For each of the study populations, the density and sex ratio of adult guppies were estimated using hand seine nets ( $3 \mathrm{~m} \times 1 \mathrm{~m}$, mesh size $=3 \mathrm{~mm}$ ) to haphazardly capture 10 different shoals from each river. To do this, we (myself and an assistant) slowly walked upstream looking for intact guppy shoals. Once a shoal was visually located, it was slowly encircled with the seine and captured in its entirely. The captured shoal was immediately placed in a bucket containing river water onshore and the collected adult guppies were counted and sexed using their visual appearance. The surface area of the river just seined was then measured using a metric tape-measure to estimate local adult 
fish density (number of guppies $/ \mathrm{m}^{2}$ ). Once a given seined shoal was so processed, we walked further upstream until another shoal was haphazardly identified and collected, and so on. We ensured that we did not seine the same sites within any river more than once on a given day. Collected male guppies were then transported by car in buckets of river water to the University of the West Indies where they were lightly anesthetised (with MS-222) and photographed individually alongside a metric ruler and under a uniform white background and lighting conditions using a Nikon Coolpix P7800 digital camera. Collected female guppies were also brought back to the laboratory for use in the experimental work discussed in Chapter 4. Digital photographs were later used to measure the body length and quantify the areas of orange and black coloration of each collected male using ImageJ (Schneider et al., 2012), as detailed in Chapter 2 (section 2.4). I measured 150 males from each population in this manner. As noted above, data on male body length and coloration for the Upper Aripo River population was obtained from a previous study (Auge et al. 2016) of this population. As this dataset contained over 600 males, a random number generator was used to select a subset of 150 individual males (the same number collected from each of the other populations examined) for analysis here. Our seining and fish collections were carried out under permit from the Fisheries Division of the Ministry of Agriculture, Land and Fisheries, Republic of Trinidad and Tobago. 


\subsubsection{Video recording and quantification of male behaviour}

Male guppies were filmed in three of the four river populations (the fourth, the Arima River, was not filmed owing to high levels of suspended sediment originating from upstream mining activity, which made it impossible to view fish in the water). Filming was done using hand-held digital video cameras (Nikon 5100 with AF-S Nikkor 28-300mm (1:3.5-5.6G ED) lens; Nikon Coolpix P7800; Canon Vixia HFM400) from vantage points either in the rivers or along their banks between 08:00 and 15:00 hrs. For each film clip, a focal male was haphazardly located in the river and then followed with the camera until it disappeared from view. Following each completed filming, myself and the other observers moved along the river to a new location before resuming filming, allowing us to minimise the likelihood of filming the same focal male repeatedly. Using video film playback, the focal male's behaviour was recorded from the first time that he was observed to enter a shoal until the last time he was observed leaving any shoal before he moved out of view (this period was defined as the total observation time). Total observation time thus did not necessarily have to include time spent within only one and the same shoal. In fact, many males moved between a number of shoals whilst they were in view during total observation time. There was no significant difference in the mean observation time among the different riverine populations (shown in Appendix 2). Focal males were considered to be shoaling when they were within four body lengths of another conspecific, as this is a commonly used inter-individual distance criterion to define shoal membership in both field and laboratory studies on the guppy (cf. Croft et al. 2003b; Croft et al. 2009a). This standard measure of fish social association is one which 
I also used in the dichotomous choice experiments in following chapters (detailed in the General Methods in Chapter 2, section 2.3). The times at which a focal male entered and left any shoal were recorded to calculate (i) shoal tenure duration, (ii) the percentage of total observation time spent shoaling, and (iii) the number of times a focal male entered a new shoal per minute (taken as a measure of shoal encounter rate). I also counted the maximum number of individuals observed in each shoal that a focal male entered as an estimate of the membership size of individual shoals. Because the size of guppy shoals changes continuously, owing to the fission-fusion nature of guppy societies (Croft et al. 2003b; Kelley et al. 2011; Wilson et al. 2014), it is not possible to have an absolute measure of shoal size.

In addition, the frequency of sigmoidal courtship displays and sneak mating attempts exhibited by individual focal males, and the number of courtship interruption events observed (either those where the focal male interrupted another male, or was interrupted), were recorded from the videos. The total number of each behaviour observed for a given focal male was then divided by the total observation time he was observed in a shoal (i.e. within 4 body lengths of a conspecific) to generate a frequency of occurrence (number of events per minute shoaling) for each behaviour for each focal male separately. 


\subsubsection{Statistical analyses}

All statistical analyses were carried out using the R statistical environment (R Core Team 2018, version 3.5.2). In all cases, I conducted an ANOVA to test whether a given dependent variable (e.g. male body length, percentage of total time spent shoaling) differed among populations. Population was always included as a fixed variable due to the limited number of levels. I examined the residuals and plotted Q-Q plots to check for the normality and homogeneity of the variance. If either of these conditions were not met, I re-analysed the data following a log-transformation. For shoal entry rate, shoaling duration and maximum shoal size, data were log-transformed to improve normality and homogeneity. If transformation did not succeed in normalising/homogenizing the residuals, then I used non-parametric Kruskal-Wallis tests to compare the data across the

different river populations, followed by Dunn tests for post-hoc multiple comparisons of means. For this reason, the Kruskal-Wallis and Dunn tests were applied to the data on male body length, male body coloration, and the frequencies of courtship displays, and sneak copulation attempts exhibited by focal males.

\subsection{Results}

\subsubsection{Male body length and coloration}

Ten seine surveys were taken in each of the four rivers between April 22 and May 3, 2015. From the seine collections, I calculated the average adult guppy fish density and 
sex ratio for each of the rivers separately (Figures 3.1 and 3.2). ANOVA results revealed no significant difference in fish density between rivers (Figure 3.1, $F=0.558, d f=3,36, p$ $=0.646)$ and a marginally significant difference in female-biased sex ratio between rivers (Figure 3.2, $F=2.851, d f=3,36, p=0.051$ ).

Body length and coloration data were obtained for 150 males collected from each of the four study populations (Figures 3.3 and 3.4). There was a highly significant difference in male body length across populations (Figure 3.3, Kruskal-Wallis test, $\mathrm{X}^{2}=$ 111.270, $d f=3, p<0.001$ ). Post-hoc pair-wise comparisons of means (Dunn test) revealed that all populations differed from one another, except the Quaré-Tunapuna paired comparison (Table 3.1), and that the Upper Aripo River males were significantly larger than males in the other populations.

I also found a significant difference in male colour score across populations (Figure 3.4, Kruskal-Wallis test, $\left.X^{2}=11.783, d f=3, p=0.008\right)$; this difference is attributable to Arima River males being significantly less colourful than males in each of the other three populations. Males from the remaining three populations (Tunapuna, Upper Aripo and Quaré) are on average similar in body coloration (Table 3.2). 




Figure 3.1. Mean \pm 1 SE adult guppy density for each of the four study populations $(n=10$ per population). Bar colour relates to predation level of the river (red= high predation, yellow $=$ intermediate, green= low). 


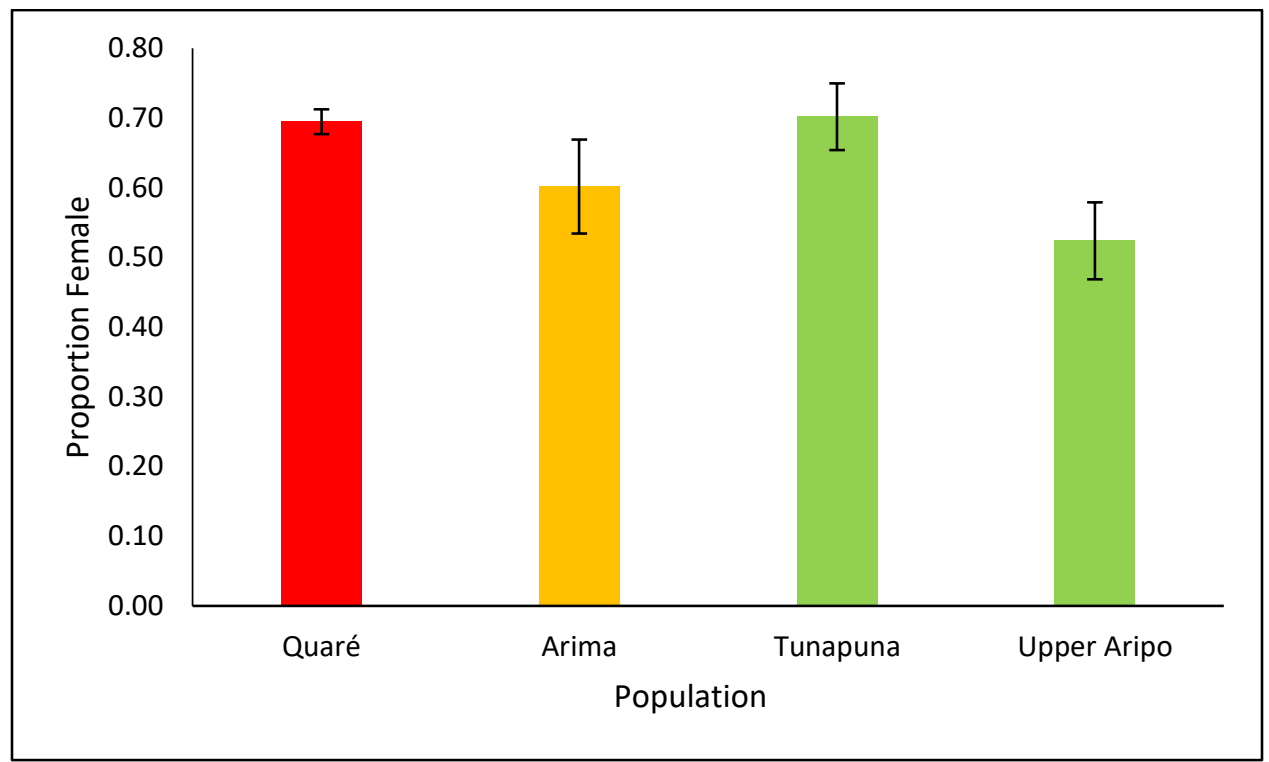

Figure 3.2. Mean \pm 1 SE proportion of adult females collected in seine samples within each of the four study populations (10 seine samples per population). Bar colour corresponds to predation level as in Figure 3.1. 


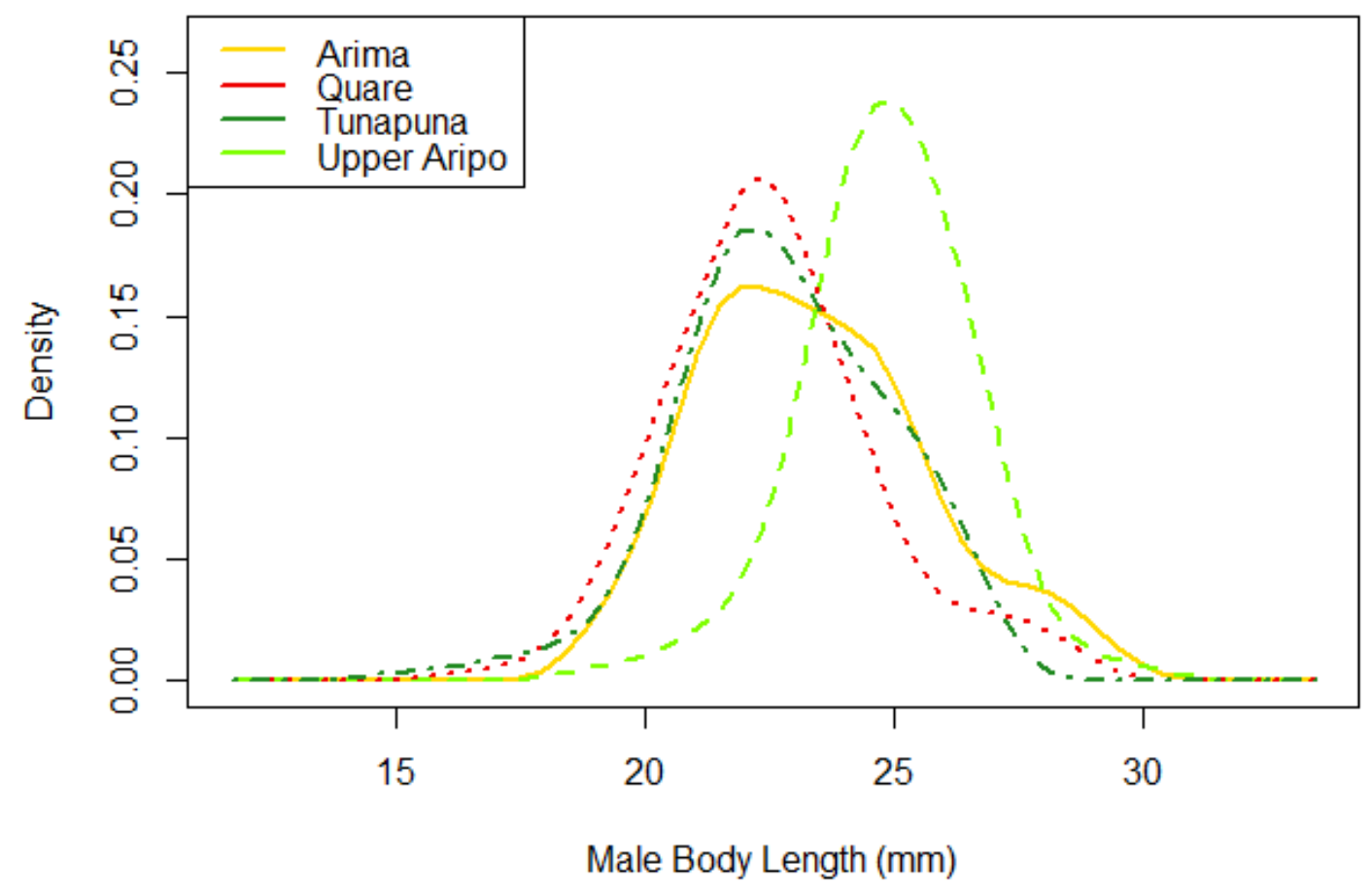

Figure 3.3. Density distribution of body length measures of free-ranging male guppies collected by seining in each of the four study populations ( $n=150$ males per population). Line colour indicates population predation level (as in Figure 3.1). 
Table 3.1. Results of a post-hoc Dunn test comparing male guppy total body length data across the four study populations (Quaré: high-predation; Arima: intermediatepredation; Tunapuna \& Upper Aripo: low-predation) in a pairwise manner. Boldfaced pvalues are statistically significant.

\begin{tabular}{|c|c|c|c|}
\hline & Arima & Upper Aripo & Quaré \\
\hline Upper Aripo & $\mathbf{p}<0.001$ & & \\
\hline Quaré & $\mathbf{p}<0.001$ & $\mathbf{p}<0.001$ & \\
\hline Tunapuna & $\mathbf{p}=0.03$ & $\mathbf{p}<0.001$ & $\mathbf{p}=0.087$ \\
\hline
\end{tabular}




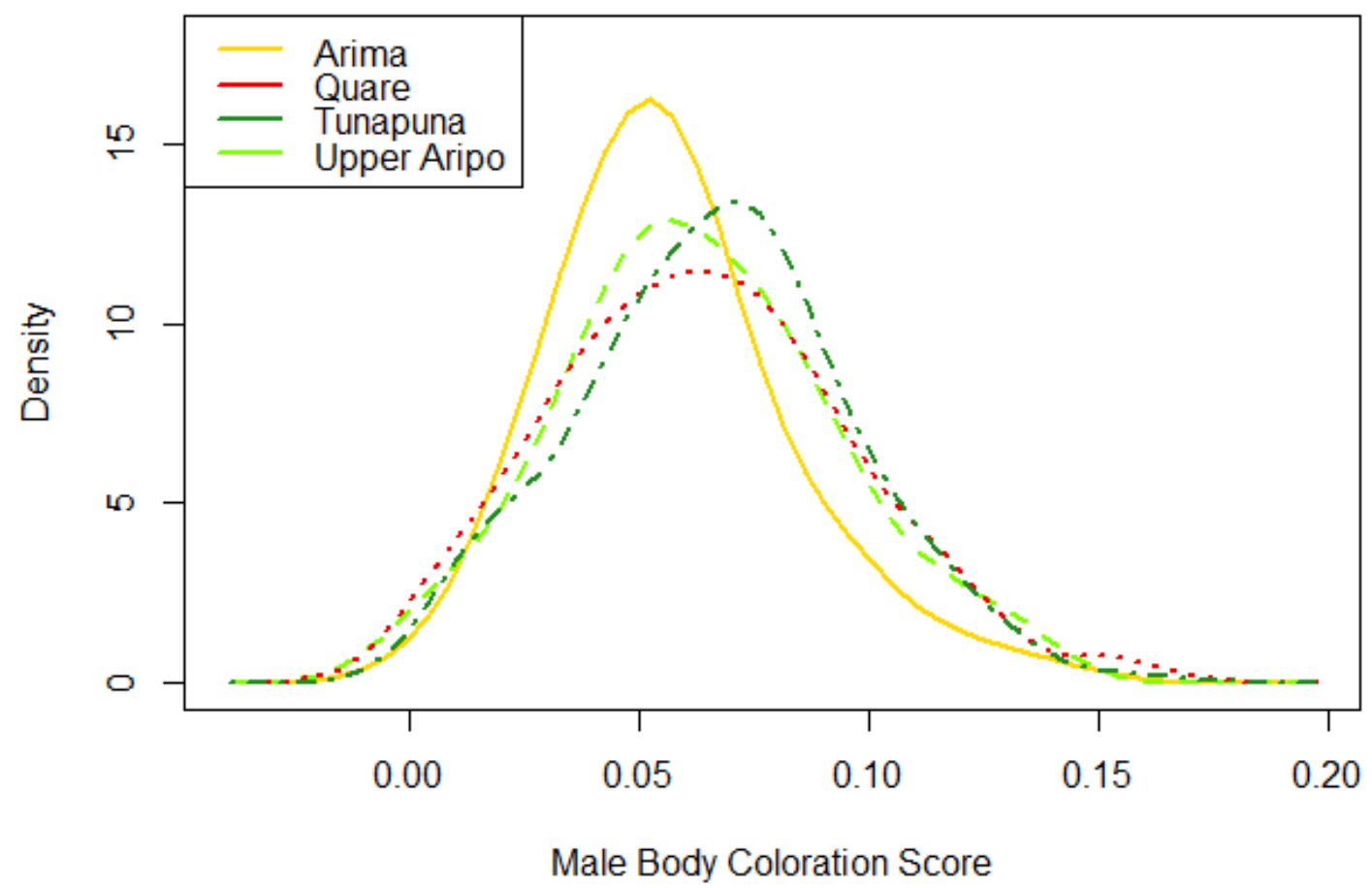

Figure 3.4. Density distribution of body coloration score (proportion of a male's body covered with orange and black colours) of free-ranging male guppies collected by seining in each of the four study populations ( $n=150$ males per population). Line colour indicates population predation level (as in Figure 3.1). 
Table 3.2. Results of a post-hoc Dunn test comparing male guppy body coloration scores across the four study populations (Quaré: high-predation; Arima: intermediatepredation; Tunapuna \& Upper Aripo: low-predation) in a pairwise manner. Boldfaced pvalues are statistically significant.

\begin{tabular}{|c|c|c|c|}
\hline & Arima & Upper Aripo & Quaré \\
\hline Upper Aripo & $\mathbf{p}=\mathbf{0 . 0 1 3}$ & & \\
\hline Quaré & $\mathbf{p}=\mathbf{0 . 0 0 9}$ & $\mathrm{p}=0.445$ & \\
\hline Tunapuna & $\mathbf{p}<\mathbf{0 . 0 0 1}$ & $\mathrm{p}=0.132$ & $\mathrm{p}=0.164$ \\
\hline
\end{tabular}




\subsubsection{Male shoaling and sexual behaviours}

A total of 64 focal males were video-recorded in three of the study populations (Upper Aripo, $n=33$; Tunapuna, $n=16$; Quaré, $n=15$ ) and a number of different social and sexual behaviours were quantified from the video clips for each focal male (see section 3.2.2 above). Courtship interruption behaviour was excluded from the analysis as it did not occur sufficiently frequently for any meaningful statistical analysis to be performed; only 5 of the 64 observed focal males had their courtship displays interrupted by a rival male at least once, and only 6 of 64 focal males were observed to be courtship interrupters on at least one occasion.

None of the measures of social (shoaling) behaviours varied significantly between populations (Figure 3.5), as shown by ANOVAs. More specifically, there were no significant differences across populations in the percentage of total observation time focal males spent shoaling $(F=2.210, d f=2,61, p=0.118)$, the rate at which focal males entered new shoals or shoal encounter rate (following a log-transformation, $F=2.016$, df $=2,61, p=0.142$ ), average time a focal fish spent within a shoal or shoal tenure (following a log-transformation, $F=0.279, d f=2,61, p=0.758)$, nor in the maximum size of shoals that the focal males had joined (following a log-transformation, $F=0.841, d f=2,61, p=$ 0.436). 


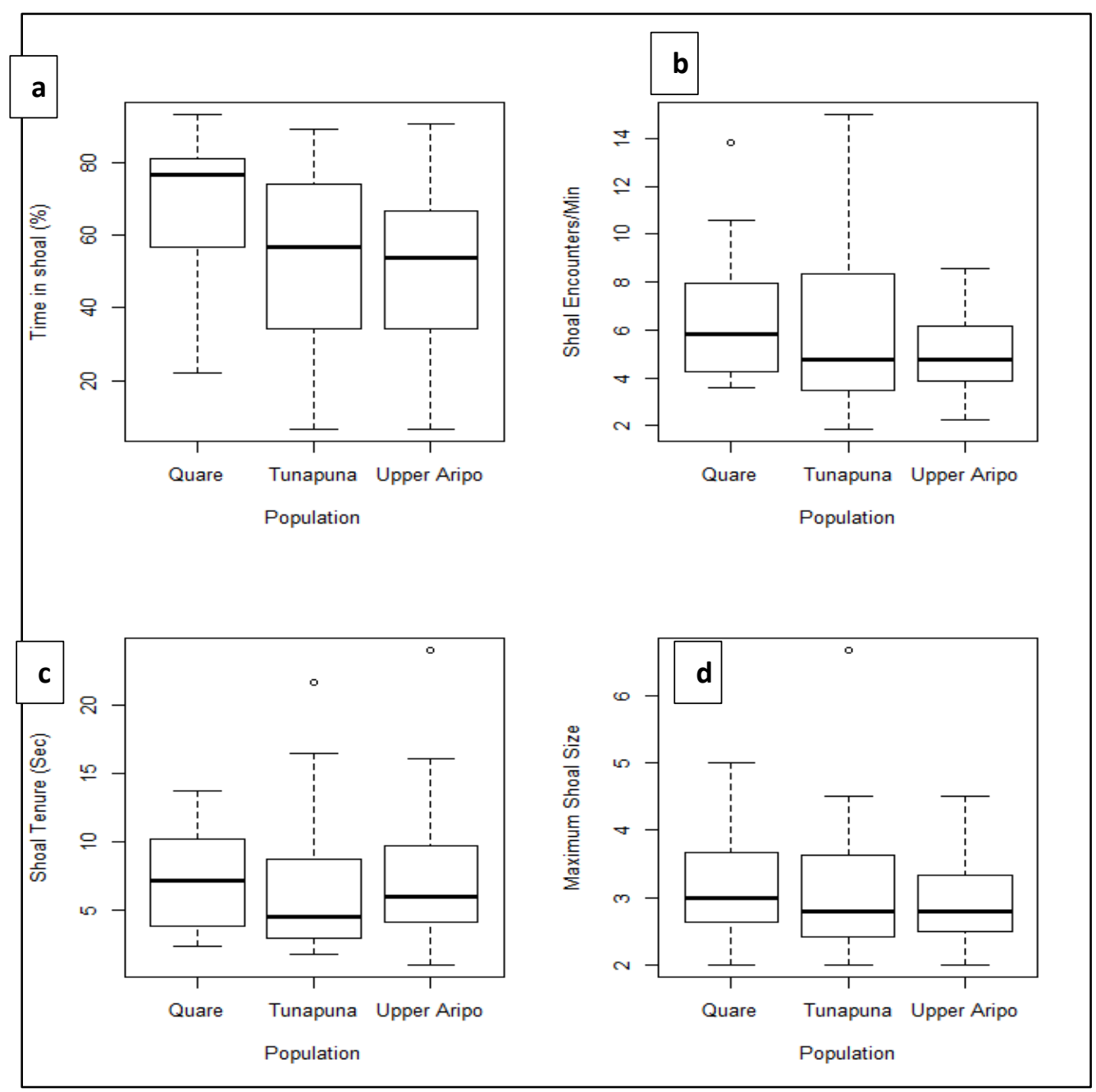

Figure 3.5. Box plots (showing median and inter-quartile intervals, with dots indicating outliers) of the shoaling behaviour metrics recorded from video clips of focal males across three populations (Quaré: high-predation; Tunapuna \& Upper Aripo: low-predation). a) Amount of time focal males spent shoaling (within 4 body lengths of a conspecific) as a percentage of total observation time for individual shoals. b) Rate at which a focal male entered new shoals per minute of total observation time (= shoal encounter rate). c) Shoal tenure duration (time that focal male remained in a shoal) in seconds. d) Maximum number of individuals in a shoal containing a focal male. 
The two sexual behaviours that I was able to quantify and analyse were the number of courtship displays and the number of sneak copulation attempts per minute shoaling (Figure 3.6). Male courtship frequency varied significantly among populations (Figure 3.6a, Kruskal-Wallis test, $X^{2}=9.411$, df $=2, p=0.009$ ); this difference was attributable to the significantly lower courtship display rates of males in the Upper Aripo population compared to males in the other two populations, which did not differ significantly from each other (Table 3.3a). Similarly, the frequencies of male sneak copulation attempts varied significantly among populations (Figure 3.6b, Kruskal-Wallis test, $\mathrm{X}^{2}=7.843, \mathrm{df}=2, \mathrm{p}=0.020$ ); this difference was attributable to the significantly higher frequency of sneak copulation attempts by males in the Quaré population compared to males in the other two populations, which did not differ significantly from each other (Table 3.3b). 


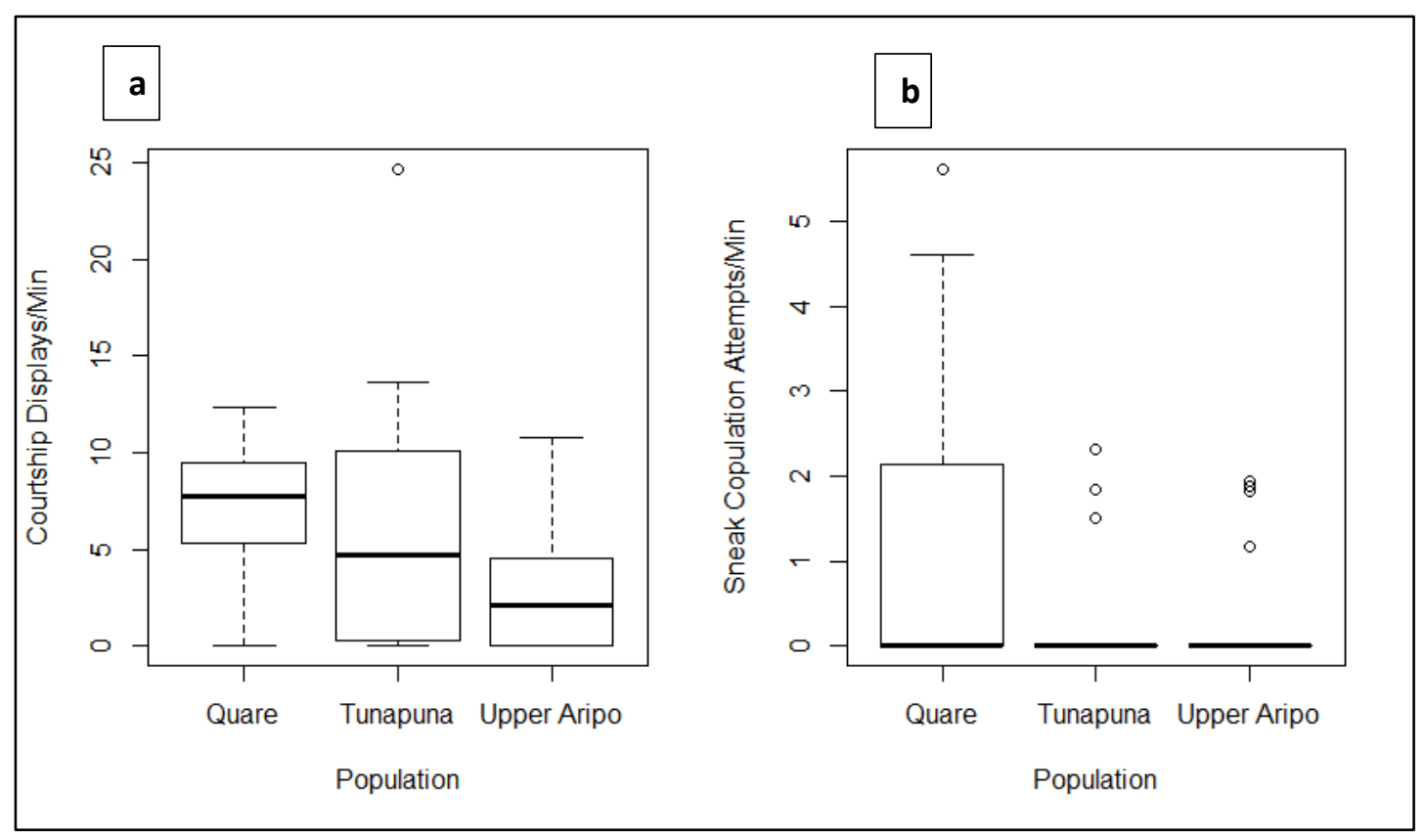

Figure 3.6. Box plots (showing the median and inter-quartile intervals, with dots indicating outliers) of the rate of sexual behaviours of focal males recorded during video analysis across three study populations (Quaré: high-predation; Tunapuna \& Upper Aripo: lowpredation). a) Number of courtship displays performed by focal males per minute spent shoaling, and b) Number of sneak copulation attempts performed by focal males per minute spent shoaling. 
Table 3.3. Results of a post-hoc Dunn test comparing (a) the mean rate of focal male courtship displays and (b) the mean rate of focal male sneak copulation attempts across the three study populations (Quaré: high-predation; Tunapuna \& Upper Aripo: lowpredation) in a pairwise manner. Boldfaced p-values are statistically significant.

a) Focal male courtship displays

\begin{tabular}{|c|c|c|}
\hline & Quaré & Tunapuna \\
\hline Tunapuna & $p=0.163$ & \\
\hline Upper Aripo & $\mathbf{p}=\mathbf{0 . 0 0 2}$ & $\mathbf{p}=\mathbf{0 . 0 3 4}$ \\
\hline
\end{tabular}

b) Focal male sneak copulation attempts

\begin{tabular}{|c|c|c|}
\hline & Quaré & Tunapuna \\
\hline Tunapuna & $p=0.025$ & $p=0.29$ \\
\hline Upper Aripo & $p=0.002$ & \\
\hline
\end{tabular}




\subsection{Discussion}

Neither adult guppy density (Figure 3.1) nor sex ratio (Figure 3.2), estimated from seine surveys, differed significantly among the study populations. It has previously been shown that guppy populations experiencing low predation intensity tend to have a more female-biased adult sex ratio, possibly due to males being more frequently targeted by weaker predators such as A. hartii (McKellar et al. 2009; McKellar \& Hendry 2011), or because of the lower productivity of such rivers (McKellar et al. 2009). Although sex ratio can potentially affect the courtship rate of male guppies at least in the laboratory (Jirotkul 1999), my findings here suggest that adult guppy density and sex ratio are unlikely to have significantly affected the behaviour of free-ranging focal male guppies observed in each of my study populations.

When considering male phenotype, I found that there was a significant difference in both male body length (Figure 3.3) and coloration (Figure 3.4) across the study populations. Males from the low-predation population of the Upper Aripo River were on average longer than those from the other populations, which corroborates previous findings showing that male guppies are larger in low-predation environments compared to high-predation ones (Endler 1995). I also found that males from the intermediatepredation Arima River population had the lowest level of body coloration on average. This is counter to my initial prediction that males from the high-predation Quaré population would be the least colourful, owing to the increased individual risk of predation associated with conspicuous body coloration (Endler 1980, 1983, 1995; Godin \& 
McDonough 2003). An alternative potential explanation for such inter-population differences in male guppy coloration (particularly orange pigmentation) is that carotenoids are diet-limited, and the levels of environmental carotenoid available to guppies can vary with the amount of canopy cover over a river, leading to populationlevel differences in average male guppy coloration (Grether et al. 1999).

An additional alternative possibility, as noted earlier in Section 3.3.2, is that the water at the site sampled in the Arima River was full of suspended sediment originating from surface runoff from longstanding quarry mine operations upstream, rendering the river water very turbid and reducing underwater visibility. This increased turbidity is likely to have reduced selection for bright coloration in male guppies, as visual cues presumably become less important in mate choice in such an environment. Over the course of multiple generations, such high turbidity may have caused a population-level shift towards less colourful males, as seen in cichlid fish species of Lake Victoria (Maan et al. 2010). It has also been noted previously that turbidity can lead to weaker predation responses and greater dispersal of shoals in guppies (Borner et al. 2015; Kimbell and Morrell 2015), which suggests that had I been able to obtain behavioural data for Arima River males, they may have shown weaker social behaviours than males in the other three study populations. A recent study of Arima River male guppies (Ehlman et al. 2018) also suggests that I would have likely observed an increased compensatory rate of sexual behaviour when males did encounter females.

I initially hypothesised that there would be a difference in the population mean of shoaling behaviour measures for focal males in the different study populations, with 
males from high-predation populations spending more time in shoals and moving less frequently between shoals (cf. Seghers 1974; Magurran \& Seghers 1991; Botham et al. 2008; Kelley et al 2011). I also expected that these males would be found in larger shoals (associating with more individuals) than those from low-predation populations, in order to dilute their risk of being predated upon (Herbert-Read et al. 2017). However, I did not find a significant difference between riverine populations in any of the shoaling behaviour measures quantified here (Figure 3.5).

Finally, I also hypothesised that there would be significant differences in the sexual behaviours of males among the study populations, with males in low-predation populations performing courtship displays at a greater rate and males in the highpredation population (Quaré River) exhibiting a higher level of sneak copulation attempts. Previous studies have shown that male guppies favour sneak copulation under increased predation risk, with males from high-risk populations particularly sensitive to the presence of predators (Luyten \& Liley 1985; Endler 1987; Magurran \& Seghers 1990, 1994b). I actually observed the lowest rate of courtship displays in the low-predation Upper Aripo guppies (Figure 3.6, Table 3.3), which might be explained by the fact that many of the focal males observed in this population spent a significant amount of time feeding, which would conflict with time available for courting females (Dussault \& Kramer 1981). Male guppies have also been shown experimentally to favour foraging over courtship (Abrahams 1993). The sites where I filmed guppies in the Upper Aripo River appeared to be less productive, with poorer quality food resources, which might explain the apparent shift to increased foraging and decreased courtship. Food availability has 
been shown to affect courtship behaviour and male-male interactions in the guppy (Kolluru \& Grether 2005; Kolluru et al. 2007). Previous studies have shown that environmental factors such as level of canopy cover can affect the productivity of a river, and that high-predation level rivers in Trinidad are often also the most productive in terms of food for guppies (Grether et al. 2001; Reznick et al. 2001). This means that guppies in these environments are able to allocate more resources to reproduction (Reznick et al. 2001), which may explain why males in my high-predation population (Quaré River) exhibited greater mating effort (courtship and sneak copulation attempt) compared to males in the other populations. Such consequences can also be seen in other species, such as the tawny owl (Strix aluco), where predation risk and food availability interact to affect the individual breeding propensity (Hoy et al. 2016).

In addition, it should be noted that males from high-predation populations are most likely to alter their behaviour in the presence of predators, and it is also known that males in these populations preferentially seek out shallower water during the day to minimise their risk of predation (Croft et al. 2003b, 2004a, 2006b). In order to be able to film the movement and behaviour of males for the current study (this chapter), I was primarily observing males in shallow waters in my study river populations and, as such, their behaviours may not have been strongly affected by the overall ambient risk of predation locally, as they presumably considered themselves to be in relatively safe habitats compared to deeper waters, leading to their exhibiting more conspicuous reproductive behaviour. 
I did, however, find that the high-predation Quaré River males performed significantly more stealth copulation attempts than males from the low-predation populations. This fits with my a priori prediction that this behaviour would be most common in riskier environments, as males seek to minimise the likelihood of being detected and predated whilst maximising their reproductive chances (Endler 1987; Magurran \& Seghers 1990, 1994b; Godin 1995). It is known that generally courtship displays in male guppies lead to greater mating success as more sperm is transferred during these solicited matings than in forced copulations (Pilastro \& Bisazza 1999), but their greater conspicuousness may have rendered courtship displays to be prohibitively costly in high-predation contexts.

Though predation level is clearly an important influence on the behaviour, morphology and life history of the Trinidadian guppy, it is not the only factor which can affect the frequency and type of reproductive tactic employed by males (see Endler 1995 for an overview). It has been shown previously that light levels in the environment can affect mating tactics employed by males, with males performing sigmoidal courtship displays at high light intensities and switching to sneak copulation when light levels are low (Chapman et al. 2009). In addition, studies on the effects of environmental pollutants, such as agricultural runoff (herbicides, cattle growth hormone etc.), have demonstrated that pollutants can alter the courtship behaviour of male guppies (Shenoy 2012; Bertram et al. 2015; Zhang et al. 2019). Such potential pollution was not considered in the current study. As the rivers in Trinidad become increasingly impacted and less pristine as a result of human activities, there is an increased likelihood that such anthropogenic factors will 
adversely affect the behaviour and survival of wild guppies. Further studies should make use of a number of replicate rivers from each predation level in order to better understand the effects of predation on these factors.

It should be noted that my observations and findings reported in this chapter represent a snapshot in time and location. It is well documented that demographic factors, such as fish density and sex ratio, can vary between years and sometimes even within years relating to events such as drought or flood (e.g. Pettersson et al. 2004; Magurran 2005). To illustrate this, I had intended to include data from a fifth population (an intermediate-predation risk population in the Lopinot River) in this analysis. However, due to seasonal drought conditions, the water level in this river was extremely low at the time of my field fish observations, and the guppies were restricted to small and very shallow isolated pools where they occurred in such high densities that it was impossible to use them in this study as they were never more than four body-lengths away from another guppy and were therefore always within a shoal according to my definition. Such extremely high guppy densities also meant I was not able to track individual focal males for more than a few seconds to quantify their behaviour before losing them from view. In addition, there is significant spatial heterogeneity in environmental conditions within rivers, and so it would be incorrect to assert that what I observed at my sites would apply throughout the entirety of the river. The current study does nonetheless provide a comparative (albeit limited) assessment of, and insights into, the social environment experienced by wild male guppies and their natural social and sexual behaviour among populations that have evolved under different predation regimes. It also further 
highlights, as many other investigators have previously (reviewed in Endler 1995; Houde 1997; Magurran 2005), the need to consider population of origin and ambient predation level when studying the behaviour, morphology and life history of the Trinidadian guppy. Though the findings here are not particularly novel, they nonetheless form a vital baseline snapshot of the phenotypic and behavioural traits present in free-swimming male guppies in wild populations that I will be studying in greater depth in the following chapters. They also allow for future computational modelling studies of the movement dynamics of guppies in these populations (following Auge et al. 2016).

Whilst this work focused on the movements of focal male guppies between shoals and their tenure within shoals, it does not inform about the identity of shoaling partners. Whilst there was apparently no significant variation in the amount of time focal males spent shoaling among rivers, I was not able to identify which individuals they were associating with during this time as the free-ranging fish were not tagged in any way and it was impossible to reliably identify individual males using their natural coloration patterns or body length from the video films, which were taken looking down at the freeranging fish. Although unknown, it is possible that different types of male conspecifics (in terms of sex, age, colour, length or personality) may have been favoured by wild focal males as social partners in the different populations. The following chapters will investigate further the social decisions made by focal male guppies and how their phenotypic traits (body length and coloration) and those of potential social partners affect such decisions. 


\section{Chapter 4}

\section{Dangerous Liaisons:}

Effects of females and population predation risk on male-male social associations in the Trinidadian guppy 


\subsection{Abstract}

In social species, the choice of same-sex social associates can have important consequences for both survival and reproductive success. Here, I used a dichotomouschoice paradigm to test for male shoaling preferences in wild Trinidadian guppies originating from three natural populations differing in predation pressure. I offered adult focal males a choice between two shoals of conspecifics from their source population, which were comprised of males that were either more or less sexually attractive than themselves based on their relative body length and coloration, both in the presence and absence of females. I found that, in the presence of females, males did not show a preference for either shoal regardless of conspecific male phenotype. However, in the absence of females, males from all populations tested preferred to socially associate with the shoal containing males that were more attractive than themselves. By preferentially associating with more sexually attractive male conspecifics, focal males might increase their mating opportunities because the latter males are more likely to attract receptive females than themselves. Alternatively, it could be that body size and coloration are indicative of foraging ability, and that focal males may enhance their own foraging success by preferentially associating with larger and more colourful conspecific males, who might be better at locating good food patches in highly heterogeneous stream habitats, when not sexually pursuing females. 


\subsection{Introduction}

Whilst some social species exhibit stable long-term social associations, many others form social associations that are temporary and which can change based on any number of factors, including predation risk, reproductive drive and internal factors such as hunger. Fluctuating groupings such as these are known as fission-fusion societies (Couzin \& Laidre 2009), where the composition of a group constantly changes as individuals join (fusion) and leave (fission) the group. This type of society occurs in a wide variety of species, including bats (Kerth \& Konig 1999), macaques (Sueur et al. 2010), ungulates (Renan et al. 2018), humans (Aureli et al. 2008), and the Trinidadian guppy (Croft et al. 2003b).

Within these shifting aggregations, individuals regularly make decisions about which conspecifics they associate with (Krause \& Ruxton 2002; Ward \& Webster 2016) and a number of different factors will affect these decisions. For example, male house finches (Carpodacus mexicanus) select their social associates by choosing to join particular flocks which maximise their relative sexual attractiveness prior to the breeding season when compared to others (Oh \& Badyaev 2010). In contrast, in the presence of predators, it may be prudent for individuals to associate with others that are as phenotypically similar to themselves as possible to minimise their individual risk of predation. The "oddity effect" is a phenomenon whereby predators preferentially target individuals which stand out from the majority in some way, which therefore selects for 
phenotypic similarity within a given group of individuals (Mueller 1971; Landeau \& Terborgh 1986; reviewed in Krause \& Ruxton 2002; Ward \& Webster 2016).

Social association decisions may be based on the physical phenotype (e.g. Krause et al. 1996; McRoberts \& Bradner 1998; Hoare et al. 2000a; Croft et al. 2005; Blakeslee et al. 2009; Jones et al. 2010) or even personality (e.g. Johnson et al. 2017; Brask et al. 2019) or other behavioural characteristics (Croft et al. 2005, 2009b) of the interacting individuals. For example, in the Wytham Woods (Oxfordshire, UK) population of great tits (Parus major), males tend to flock with others of a similar personality type (relating to the individual's exploration rate) to themselves in winter (Aplin et al. 2013). A later study similarly found that males are assorted positively by personality during the breeding season, preferentially breeding closer to others of similar personality types (Johnson et al. 2017). Neither study found any evidence of females exhibiting this assortative association (Aplin et al. 2013; Johnson et al. 2017), highlighting that preferences expressed by one sex may not always be evident in the other sex within a species. In other species (such as the guppy), it is the females that show most evidence of assortative association, for example by body length (Croft et al. 2003b, 2005, 2009a), with less evidence for this seen in the males (Auge et al. 2016).

In this chapter, I report on my research focusing on male-male social associations based on phenotypic traits, and consider how two potentially conflicting ecological factors, namely mating opportunity and predation risk, may affect an individual's decisions regarding whom to socially associate with. I used the Trinidadian guppy as a model study species. A general introduction to the guppy is provided in Chapter 2 (section 
2.1). In brief, the guppy is a sexually dimorphic, live-bearing fish endemic to Trinidad, though it has also been widely introduced in other areas worldwide for mosquito pest control (Magurran 2005). Both males and females have multiple mates (Kelly et al. 1999; Neff et al. 2008), and both exhibit mate choice (e.g. Houde 1997; Herdman et al. 2004; Godin \& Auld 2013). Females generally prefer larger, more ornamented males (Houde 1997; Magurran 2005; Auld et al. 2016), and males favour larger, more fecund females (Herdman et al. 2004; Jeswiet et al. 2012; Godin \& Auld 2013). There is also some indication that females exhibit a preference for uncommon or rare male phenotypes (Hughes et al. 1999; Dargent et al. 2019). Male guppies utilise alternatively two different mating tactics to mate with females. They can either perform conspicuous courtship displays or attempt sneak copulations (also known as a gonopodial thrust) (Houde 1997).

As mentioned above, guppy shoals exhibit fission-fusion dynamics (Croft et al. 2003b) and, as such, this species has been used in many studies of social association (e.g. Croft et al. 2003a, b; Croft et al. 2004b; Jones et al. 2010; Brask et al. 2011; Gasparini et al. 2013; Auge et al. 2016). Previous studies focused on female social behaviour, as females are generally considered the more social sex and spend more time shoaling than males in the guppy (Magurran 2005). When offered a choice, female guppies prefer to shoal with larger females (Jones et al. 2010), and free-ranging shoals of guppies (largely comprised of females) can be assorted by body size (Croft et al. 2003b). This size-assorted shoaling may be partly due to environmental constraints (i.e. differential habitat use by individuals of different body size) rather than solely by an individual's preference for specific companions (Croft et al. 2003b). One study even suggests that female guppies are 
able to select their social environment to avoid costly sexual harassment from male guppies, with gravid (and therefore sexually non-receptive) females showing a preference for shoaling with sexually receptive females, who are more likely to be pursued by males (Brask et al. 2011).

Male guppies are more transient, regularly moving between shoals in search of mating opportunities (Croft et al. 2003a). As a result, less consideration has been given to the social associations of males, despite the fact that they will clearly also have decisions to make about their associates. As the earlier great tit example demonstrates, males and females of a species can make different decisions about optimal social associates due to the different selection pressures they experience (Aplin et al. 2013; Johnson et al. 2017). Because larger and (or) more ornamented male guppies are more attractive potential mates to females (Magurran 2005; Auld et al. 2016), smaller, less ornamented males may therefore avoid prolonged associations with such males to avoid appearing comparatively less desirable to females (Bateson \& Healy 2005; Gasparini et al. 2013). Conversely, associating with larger and more ornamented males may bring an individual into contact with shoals of females more frequently, and thus increase the likelihood of potential matings. A previous study found no evidence of assortative shoaling based on body length or coloration in male guppies, with males apparently selecting their associates randomly (Auge et al. 2016), though this study only considered individuals from one population (the low-predation Upper Aripo-Naranjo tributary in the Northern Range mountains of Trinidad). 
There is evidence of an audience effect in guppies, where male guppies alter their preference for females when other males are present (Auld \& Godin 2015), and this change in preference can be most pronounced when the audience males are larger and more colourful (more orange and black body coloration) than themselves (Auld et al. 2017). This also suggests that larger, more colourful males may be perceived as stronger sexual and sperm competitors and therefore should be less preferable shoal mates for male guppies in search of mates.

As highlighted in Chapter 2 (section 2.1), guppies in Trinidad live in geographically and genetically distinct populations that are subject to different environmental conditions, including differing predation level (Houde 1997; Magurran 2005; Croft et al. 2006b; Botham et al. 2008). Predation level is here defined as the presence/absence and attack frequency of fish predators towards adult guppies (explained further in Chapter 2, section 2.1). Guppies from high-predation risk populations exhibit different behaviours from their low-predation conspecifics (reviewed in Magurran 2005). For example, a reduction in flamboyant courtship displays and increased levels of inconspicuous, sneak copulations by males have been observed in high-risk populations (Endler 1987; Godin 1995). I also observed higher frequencies of sneak copulations in a high-predation risk riverine population (Chapter 3). Guppies from high-predation risk populations also exhibit different phenotypic characteristics, as they are often smaller and less ornamented than those from populations under lower predation pressure (Endler 1980; Magurran 1995). Comparative phenotypic data from four different populations presented in Chapter 3 also showed that the largest males were found in a low-predation population (the Upper Aripo 
River). It has previously been demonstrated that large and colourful male guppies are more conspicuous to predators and suffer greater mortality risks to predation than smaller and less colourful conspecifics (Endler 1980; Godin \& McDonough 2003; Johannson et al. 2004), which likely explains the observed reduction in body size and male ornamentation in populations that have evolved under high predation pressure.

Here, I address the relative lack of attention given to male-male social associations by comparing the behaviour wild male guppies originating from three geographically separated riverine populations. These populations are subject to different levels of fish predation, which allowed me to consider the effect of multiple factors (predation risk and mating opportunities) on male-male social associations. Focal males from each of the three populations (predation levels) were offered a choice to socially associate with either a shoal of more attractive conspecific males or a shoal of less attractive males than themselves, in the presence or absence of females. Male sexual attractiveness was assessed based on body length and the relative areas of black and orange pigmentation on a male's body (see Table 4.3 for mean body length and colour scores).

I expected that males from the low-predation risk population would have the strongest preference to socially associate with less attractive conspecifics over more attractive ones, and that this preference would be exaggerated in the presence of females, if focal males are attempting to maximise their relative sexual attractiveness to females (as in Oh \& Badyaev 2010) and minimise the risks of an audience effect (Auld et al. 2017). A study in the leaf-footed cactus bug (Narnia femorata) showed that the presence or absence of females influenced whether certain male phenotypic traits 
affected the likely outcome of male-male contests (Procter et al. 2012) and thus demonstrated how associations between conspecifics can vary depending on the presence of the opposite sex. I expected that males from high-predation risk populations would also tend to prefer to associate with less attractive conspecifics (so to maximize their relative sexual attractiveness; Bateson \& Healy 2005), but that this preference would be less pronounced than in males from low-predation populations because of the opposing selective force of greater ambient predation risk against relatively more conspicuous males (Endler 1980; Godin \& McDonough 2003; Johannson et al. 2004). This could make drab shoal mates dangerous company, as the focal male would be relatively more conspicuous in comparison and therefore potentially at greater risk of predation.

\subsection{Methods}

\subsubsection{Fish collection and maintenance}

I used hand seine nets to collect wild guppies in three different river populations in the Northern Mountain Range, Trinidad, each representing a different level of predation (as discussed in Chapter 2, section 2.1). Fish were collected from the Tunapuna River (low predation), the Arima River (intermediate predation) and the Quaré River (high predation) (Magurran 2005; Croft et al. 2006b; Botham et al. 2008). Collected guppies were placed in plastic buckets containing river water and transported by car to an aquatics laboratory at the University of West Indies, St. Augustine, where they were held in large, mixed-sex aquaria containing aged tap water $\left(24-26^{\circ} \mathrm{C}\right)$ and exposed to a $12 \mathrm{~h} \mathrm{~L}$ : 
$12 \mathrm{~h}$ D light regime (overhead fluorescent lights, supplemented with natural daylight from small windows) and fed twice daily with commercial flake food. Further details of fish care are given in Chapter 2 (section 2.2). By maintaining the guppies in a mixed-sex environment, I ensured that the vast majority of females would be inseminated and thus not sexually receptive, as most are in the wild (Houde 1997). In doing so, I standardised as much as possible the reproductive status and sexual motivation of the stimulus females in paired stimulus shoals, thus minimising the likelihood of their behaviour biasing the choice of focal males to associate with either of the paired shoals presented in any given trial.

\subsubsection{Experimental protocol and apparatus}

Focal male preference for either of two stimulus shoals simultaneously presented was tested using a dichotomous choice paradigm, commonly used in guppy behavioural choice studies (reviewed in Houde 1997). In each behavioural trial, individual focal males were offered a choice to socially associate with either of two shoals, each comprising two conspecifics from their home population, where the males within them varied in their phenotype (body size and proportion of orange/black coloration). The amount of time a focal male spent associating with a stimulus shoal is considered representative of his shoaling preference for that shoal (Jeswiet \& Godin 2011).

When selecting experimental fish for a given trial, I ensured that the focal male and the stimulus fish were selected from different test aquaria, as social familiarity and 
shoaling preferences can develop within 12 days (Griffiths \& Magurran 1997; Croft et al. 2004c) and I wished to avoid any preferential associations based on familiarity as guppies have social preferences for familiar individuals (Magurran et al. 1994; Cattelan et al. 2018). For all trials, a focal male was selected and used. This male was of intermediate length and body colour (quantified as the proportion of the body covered with black (melanin) and orange-red (carotenoid) pigment patches, excluding the tail) (Figure 4.1). Each focal male was only used once, and so was only subjected to either the femalepresent or the female-absent treatment. For each female-present trial, two stimulus males and two stimulus females were also selected (Figure 4.1, 4.2). The females were size matched (aiming for difference of $<1 \mathrm{~mm}$ ) by eye to minimise any effect of differences in their body size biasing male shoal choice, as male guppies tend to prefer larger (and potentially more fecund) females over smaller ones as mates (Herdman et al. 2004; Godin \& Auld 2013). Males were selected to be as different from each other as possible, with a less attractive (LA) stimulus male being both smaller and less colourful than the more attractive (MA) focal male (see Table 4.2). For each female-absent trials, only stimulus males were used, with two males being classified as less attractive (i.e. smaller, drabber males), and two being more attractive (large and colourful) than the focal male. In any given trial, all focal and stimulus fish originated from the same population. The type of individuals used in the paired stimulus shoals for each treatment can be seen in Figure 4.1, and sample sizes for each population and treatment are given in Table 4.1. 
a) Female-present treatment

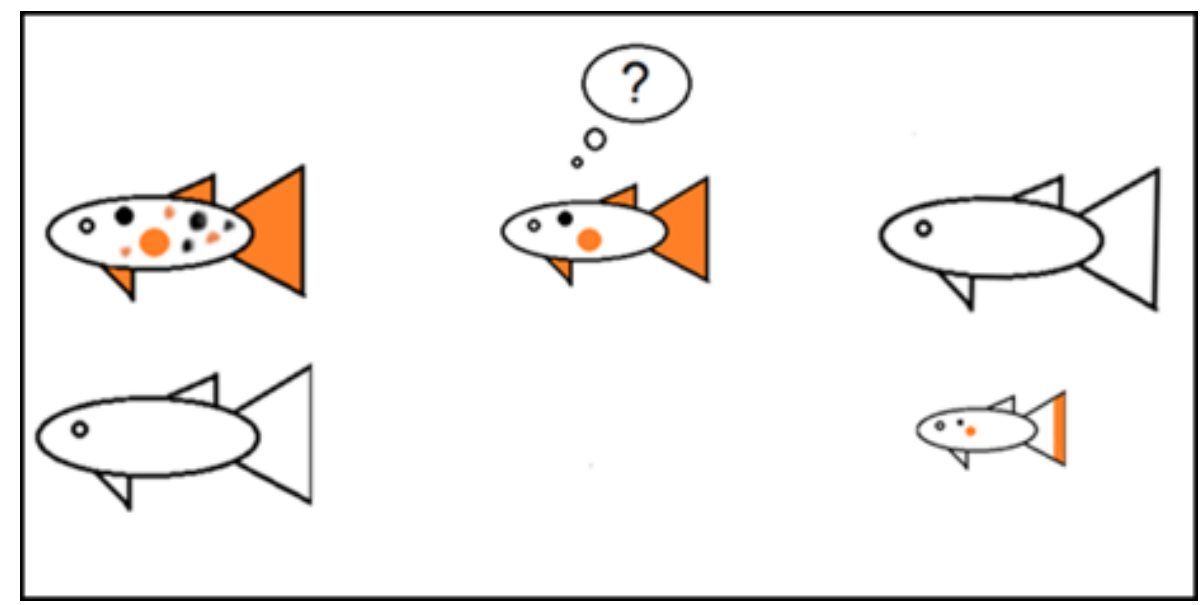

OR

b) Female-absent treatment

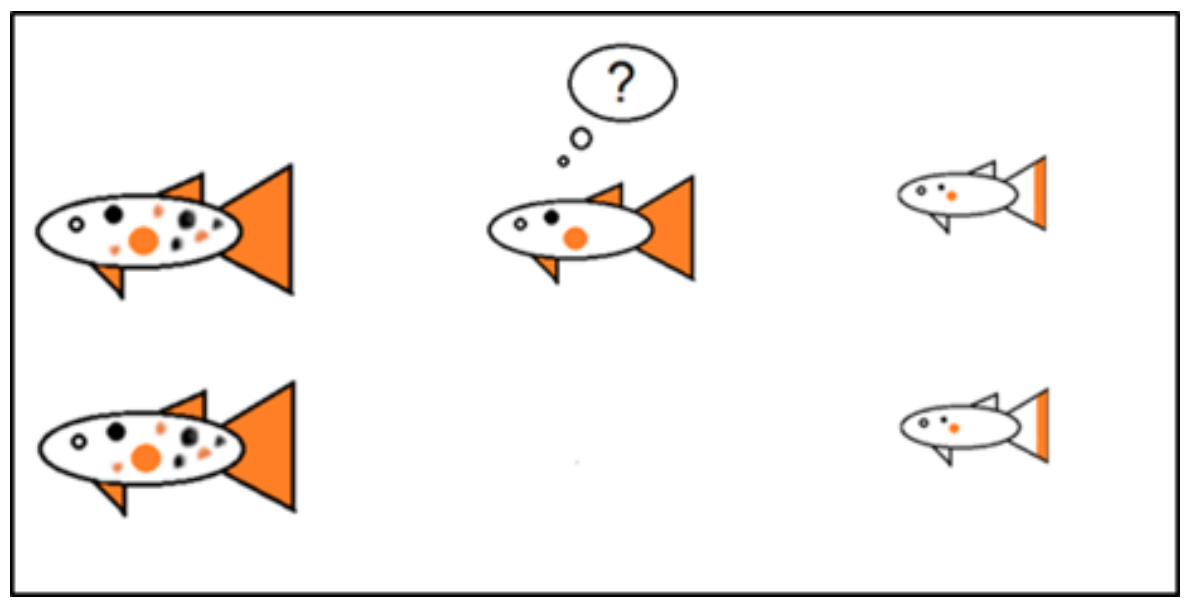

Figure 4.1. Composition of the paired stimulus shoals for a) the female present and b) female absent experimental treatments. Each focal male was only subjected to one of the two treatments. 
Table 4.1. Total number of trials (focal males) for each of the treatments and study populations (Quaré: high-predation; Arima: intermediate-predation; Tunapuna: lowpredation).

\begin{tabular}{|c|c|c|}
\hline River Populations & Females Present & Females Absent \\
\hline Quaré & 28 & 26 \\
\hline Arima & 29 & 30 \\
\hline Tunapuna & 30 & \\
\hline
\end{tabular}


A standardised experimental set-up was used for the dichotomous choice test, as described in Chapter 2 (section 2.3). Briefly, the experimental apparatus comprised a central test aquarium in which a focal male was placed. Initially, the focal fish was contained within a clear Plexiglas cylinder in the middle of the central aquarium and allowed to acclimatise for $15 \mathrm{~min}$. A smaller clear Plexiglas container was placed at either end of the central aquarium, each divided into two compartments by a clear Plexiglas barrier, and contained the stimulus fish (Figure 4.2). All outward-facing sides of the tanks were covered in brown paper to minimise any external visual disturbances to the fish during the trials. One stimulus fish was placed into each of the 4 compartments, with one side representing the less-attractive male shoal and the other the more-attractive male shoal. The side placement of the more or less attractive shoal was alternated between trials to minimise any side bias in the focal male's shoal choice. During the female-present trials, females were placed diagonally opposite each other (Figure 4.2). 


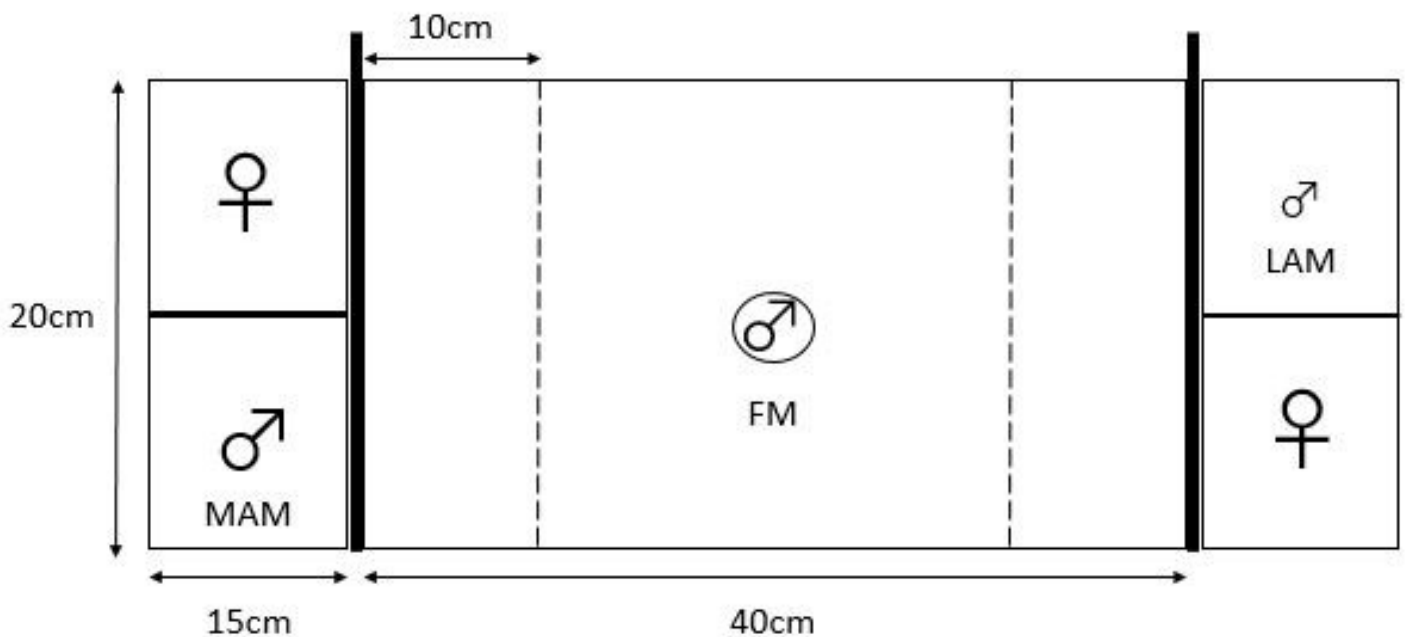

Figure 4.2: Schematic of the experimental apparatus (overhead view) showing the experimental tanks for a social association preference test. The middle aquarium contains the focal male (FM), held initially within a clear tube (shown), whilst the two end containers each hold a stimulus shoal of two fish separated by a clear divider. Here, the more attractive male (MAM) is on the left side, and the less attractive male (LAM) on the right. This figure illustrates the set-up for the female-present treatment, where two sizematched females are placed one at either end. For the female-absent treatment, the females would be replaced by a second stimulus male (of a similar phenotype to the other male in the relevant stimulus shoal) taken from the same population as the focal male. The apparatus is pictured here with the opaque screens in place, blocking the stimulus shoals from the focal male's view. These screens were removed during the acclimatisation period, prior to the start of the free-swimming choice period (as described below). 
For the first $10 \mathrm{~min}$ of the acclimatisation period, the stimulus shoal end containers were blocked from the focal male's view by opaque screens (Figure 4.2). These screens were then removed for the final 5 min of acclimatisation period to allow the focal male to view the stimulus shoals whilst still inside the central cylinder. Once the full 15 min had elapsed, the Plexiglas cylinder was removed, and the focal male allowed to swim freely in the central test aquarium and to choose to associate with either stimulus shoal. Each end of the central aquarium had a demarcated $10-\mathrm{cm}$ wide social preference zone, which corresponds to about four adult male guppy body lengths (the measure of guppy shoaling used throughout this thesis; cf. Croft et al. 2003b; 2009a). If the focal male entered this zone, he was considered to be socially associating with the adjacent shoal. This free-swimming behaviour was observed for 10 min using an HD webcam (Logitech C920 HD Pro) mounted $40 \mathrm{~cm}$ above the central aquarium and coupled to a laptop computer. Using JWatcher ${ }^{\mathrm{TM}}$ (version 0.9; Blumstein \& Daniel 2007), I recorded the movements of the focal male and the time he spent in each preference zone. After 10 $\min$, the opaque screens were replaced, and the positions of two end containers were switched to control for any potential side bias of the focal male (discussed in more detail in Chapter 2, section 2.3). Any focal male who did not sample both preference zones during the first 10-min preference test was removed and the trial was voided. The focal male was returned to the central cylinder, and the acclimatisation period was repeated. At the end of this period, the cylinder was raised and the focal male was allowed again to swim freely again and to choose between the two stimulus shoals for $10 \mathrm{~min}$. I recorded the behaviour of the focal male as during the first preference test. 
After the second 10-min preference test (with the stimulus shoals on the opposite sides to the first preference test), all fish were collected, lightly anaesthetised using MS222, individually placed on their side on a piece of white Plexiglas and digitally photographed. Their body length and body coloration (males only) were quantified from their photographs using the standard protocols detailed in Chapter 2, section 2.4. JWatcher data records were used to calculate the total amount of time the focal male spent within either preference zones, taken as a measure of his social association preference for either stimulus shoal. From these data, I calculated the total time (summed over both 10-min preference tests) that the focal male spent shoaling with the more attractive shoal for each trial separately. Each focal male was only used once, whilst stimulus males were sometimes reused (but were never used more than once daily).

\subsubsection{Statistical analyses}

All statistical analyses were carried out in the R statistical environment ( $\mathrm{R}$ Code Team 2018, version 3.5.2). First, for each population and treatment separately, I used the paired t-test to ascertain whether the stimulus males in the paired stimulus shoals differed significantly in both body length and coloration as planned (Table 4.2). Second, for the female-present treatment, I also confirmed that there was no significant difference in female body size as this could affect male preference (Table 4.3). Third, for each population and each treatment separately, I used a one-sample t-test to compare 
the proportion of time the focal male spent shoaling with the more attractive shoal against that expected by chance (i.e. 0.50 proportion of total time shoaling).

Lastly, I fitted a general linear model (model: Im (asin(TimeMA) (as.factor(Population) * as.factor(Treatment)) + DiffLength + DiffColour + FocalLength + FocalColour)) to determine the effects of population and treatment (presence/absence of females), and their interaction, as well as phenotypic traits (the focal male length and colour score, and the differences between the mean length and colour scores of males in the two stimulus groups) on the proportion of time focal males spent shoaling with the more attractive shoal (arcsine-transformed as these data are proportional). Population was included as a fixed effect rather than a random effect due to the small number of levels (3) involved. I examined the model residuals and plotted Q-Q plots to confirm the normality and homogeneity of the residuals.

\subsubsection{Ethical standards}

This research was approved by the Carleton University Animal Care Committee (protocol \#102911) and adheres to the animal use and care guidelines of the Canadian Council on Animal Care and thus the laws of Canada. Wild guppies were collected from their natural home population under permit from the Fisheries Division of the Ministry of Agriculture, Land and Fisheries, Republic of Trinidad and Tobago. All healthy fish were returned to the site of their collection in their river of origin at the end of the experiment. 


\subsection{Results}

As the current experiment presented focal male guppies with a dichotomous choice of two stimulus shoals containing conspecific stimulus males of different phenotypes (i.e. sexual attractiveness), I first had to confirm that there was in fact a significant difference in the phenotypes of the males in the paired stimulus shoals. As shown in Table 4.2, there was a significant difference in both body length and coloration between the more and less attractive stimulus males in both treatments for all three study populations, with the focal male being intermediate between the paired stimulus males in both phenotypic measures. In the female-present treatment, there was no significant difference in length between the two females presented (Table 4.3), meaning that female size should not have affected the focal male's preference for a given shoal and could therefore be omitted as a covariate from the later general linear model (Table 4.5). 
Table 4.2. Mean \pm SE total body length $(\mathrm{mm})$ and body colour score (proportion of left side covered with orange and black coloration) of focal males and stimulus males classified as more and less attractive stimulus fish. Each paired t-test compares the phenotypic measures for the paired more attractive and less attractive stimulus males used in individual trials.

\begin{tabular}{|c|c|c|c|c|c|}
\hline $\begin{array}{l}\text { Population- } \\
\text { Treatment }\end{array}$ & Trait & $\begin{array}{c}\text { More } \\
\text { attractive } \\
\text { stimulus } \\
\text { male(s) }\end{array}$ & Focal male & $\begin{array}{c}\text { Less attractive } \\
\text { stimulus } \\
\text { male(s) }\end{array}$ & Paired t-test \\
\hline \multirow{2}{*}{$\begin{array}{c}\text { Quaré River } \\
\text { Females } \\
\text { Present }\end{array}$} & Length & $24.31 \pm 0.38$ & $22.25 \pm 0.18$ & $20.66 \pm 0.28$ & $\begin{array}{c}t=7.90, d f=27 \\
p<0.001\end{array}$ \\
\hline & Colour & $0.075 \pm 0.0056$ & $0.070 \pm 0.005$ & $0.041 \pm 0.0041$ & $\begin{array}{c}\mathrm{t}=5.45, \mathrm{df}=27, \\
\mathrm{p}<0.001\end{array}$ \\
\hline \multirow{2}{*}{$\begin{array}{c}\text { Quaré River } \\
\text { Females } \\
\text { Absent }\end{array}$} & Length & $24.41 \pm 0.22$ & $22.39 \pm 0.21$ & $20.78 \pm 0.17$ & $\begin{array}{c}t=15.96, d f=25 \\
p<0.001\end{array}$ \\
\hline & Colour & $0.078 \pm 0.0047$ & $0.074 \pm 0.006$ & $0.042 \pm 0.0037$ & $\begin{array}{c}t=5.67, d f=25 \\
p<0.001\end{array}$ \\
\hline \multirow{2}{*}{$\begin{array}{c}\text { Arima River } \\
\text { Females } \\
\text { Present }\end{array}$} & Length & $25.33 \pm 0.41$ & $23.25 \pm 0.25$ & $21.34 \pm 0.19$ & $\begin{array}{c}t=8.66, d f=28, \\
p<0.001\end{array}$ \\
\hline & Colour & $0.072 \pm 0.0053$ & $0.059 \pm 0.004$ & $0.045 \pm 0.0036$ & $\begin{array}{c}\mathrm{t}=4.16, \mathrm{df}=28, \\
\mathrm{p}<0.001\end{array}$ \\
\hline \multirow{2}{*}{$\begin{array}{c}\text { Arima River } \\
\text { Females } \\
\text { Absent }\end{array}$} & Length & $25.58 \pm 0.23$ & $23.19 \pm 0.23$ & $21.43 \pm 0.18$ & $\begin{array}{c}\mathrm{t}=15.41, \mathrm{df}=27 \\
\mathrm{p}<0.001\end{array}$ \\
\hline & Colour & $0.066 \pm 0.0039$ & $0.062 \pm 0.004$ & $0.042 \pm 0.0033$ & $\begin{array}{c}t=5.70, d f=27, \\
p<0.001\end{array}$ \\
\hline \multirow{2}{*}{$\begin{array}{l}\text { Tunapuna } \\
\text { River } \\
\text { Females } \\
\text { Present } \\
\end{array}$} & Length & $24.76 \pm 0.26$ & $22.34 \pm 0.13$ & $20.86 \pm 0.27$ & $\begin{array}{c}t=13.84, d f=29 \\
p<0.001\end{array}$ \\
\hline & Colour & $0.083 \pm 0.0040$ & $0.069 \pm 0.005$ & $0.043 \pm 0.0047$ & $\begin{array}{c}t=5.87, d f=29, \\
p<0.001\end{array}$ \\
\hline \multirow{2}{*}{$\begin{array}{c}\text { Tunapuna } \\
\text { River } \\
\text { Females } \\
\text { Absent }\end{array}$} & Length & $24.60 \pm 0.21$ & $22.60 \pm 0.21$ & $20.67 \pm 0.19$ & $\begin{array}{c}\mathrm{t}=16.65, \mathrm{df}=29 \\
\mathrm{p}<0.001\end{array}$ \\
\hline & Colour & $0.078 \pm 0.0038$ & $0.069 \pm 0.004$ & $0.041 \pm 0.0039$ & $\begin{array}{c}t=6.19, d f=29, \\
p<0.001\end{array}$ \\
\hline
\end{tabular}


Table 4.3 Results of paired t-tests comparing the body lengths of the two stimulus females (one in each of the paired stimulus shoals) presented during the female-present trials. As indicated by $p$ values of $>0.05$, there was no significant difference between the sets of females, suggesting female body length should not have had a significant effect on focal male shoal choice.

\begin{tabular}{|c|c|c|c|}
\hline Population & t & d.f. & p \\
\hline Quaré River & 0.183 & 27 & 0.856 \\
(high-predation) & & & \\
\hline Arima River & 0.045 & 28 & 0.964 \\
(intermediate-predation) & & & \\
\hline Tunapuna River & -1.504 & & 0.144 \\
& & & \\
(low-predation) & & & \\
\hline
\end{tabular}


Across all three populations, focal males had a significant preference for socially associating with more attractive male conspecifics over less attractive ones, but only in the absence of females (Table 4.4, Figure 4.3). When females were present, they did not show any preference for either shoal and appeared to associate randomly with other guppies.

I then fitted the data to a more inclusive general linear model to test for the effects of experimental treatment (i.e. the presence or absence of females), population of origin, and phenotypic traits (length and colour of focal males, and the differences in length and colour between the males in the stimulus shoals), on the proportion of time focal males spent associating with the more attractive shoal (these data were arcsine transformed; see Table 4.5 for full model). The only significant main factor affecting the shoaling decisions of focal males was the experimental treatment (i.e. the presence or absence of females) ( $F=14.050, d f=1,161, p<0.001 ;$ Table 4.5). There was no significant difference in the shoaling behaviour of focal males between the different populations $(F=1.387$, $d f=2,161, p=0.253$; Table 4.5), nor any significant effect of phenotypic traits, either of the focal fish or of the differences in phenotype between the stimulus shoals, on the proportion of time spent with the more attractive shoal (Table 4.5). 


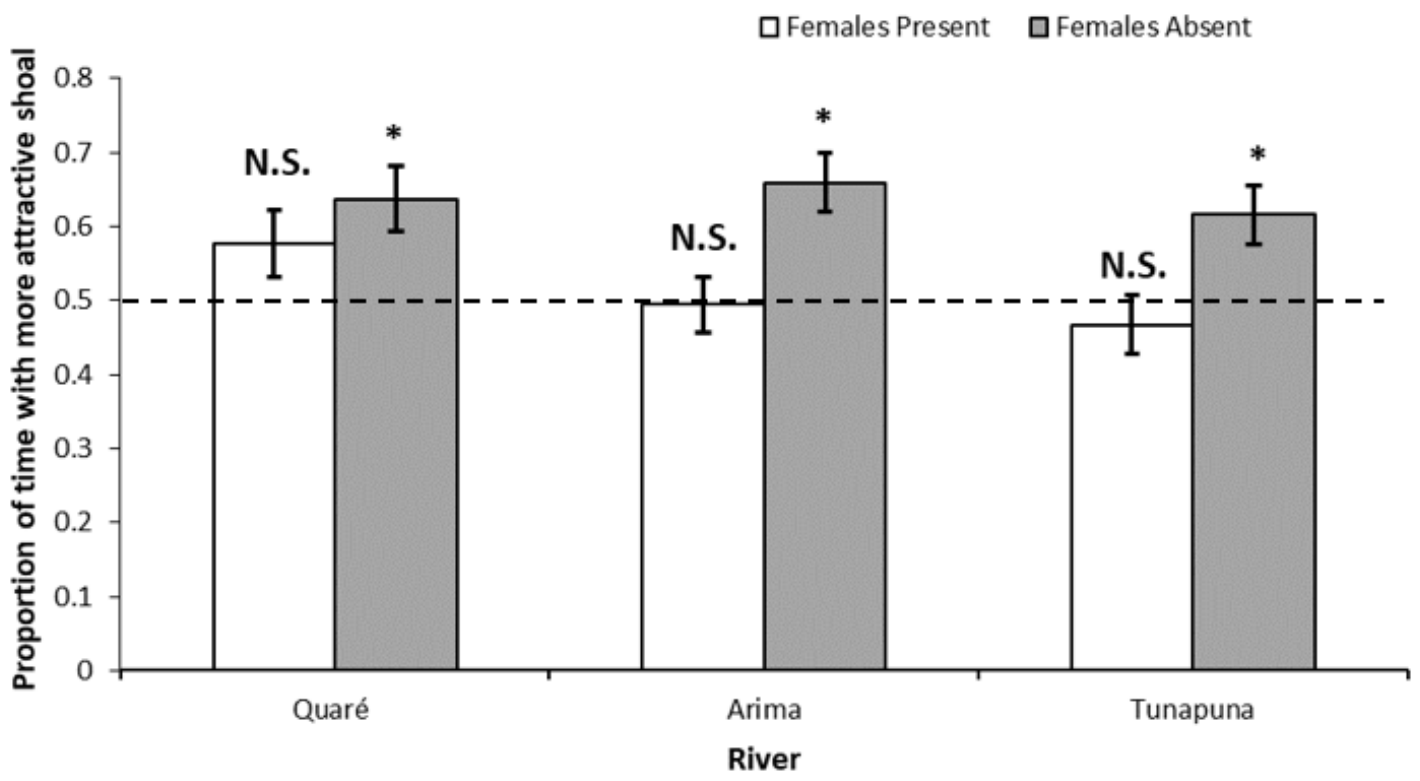

Decreasing Population Predation Risk

Figure 4.3. Mean \pm SE proportion of time focal males spent associating (shoaling) with the shoal containing the more attractive male(s) across the three study populations (Quaré: high-predation; Arima: intermediate-predation; Tunapuna: low-predation), when females were present in the stimulus shoals (open bar) and when females were absent (filled bar). An asterisk $(*)$ above a mean denotes a choice preference for the more attractive shoal that is significantly greater than that expected from chance (indicated by the horizontal stippled line at 0.50 ; see details of the one-sample t-tests in Table 4.6). No significant different from chance is denoted by N.S. 
Table 4.4. Results of one-sample t-tests comparing the proportion of time the focal male spent shoaling with the more attractive shoal against the proportion $(=0.50)$ expected by chance (i.e. no preference). $\mathrm{P}$ values in boldface indicate significant differences, and therefore a preference for either stimulus shoal.

\begin{tabular}{|c|c|c|c|c|}
\hline Population & Treatment & t & d.f. & P \\
\hline \multirow{2}{*}{ (high-predation) } & Females Present & 1.684 & 27 & 0.104 \\
\cline { 2 - 5 } & & & 25 & $\mathbf{0 . 0 0 5}$ \\
\hline Arima River & Females Present & -0.158 & 28 & 0.876 \\
(intermediate-predation & Females Absent & 3.983 & 27 & $<\mathbf{0 . 0 0 1}$ \\
\cline { 2 - 5 } & & & & 0.414 \\
\hline Tunapuna River & Females Present & -0.828 & 29 & $\mathbf{0 . 0 0 7}$ \\
\cline { 2 - 5 } (low-predation) & Females Absent & 2.904 & 29 & \\
\hline
\end{tabular}


Table 4.5. ANOVA table and parameter estimates of the general linear model testing for the effects of population, treatment (female presence/absence) and phenotypic traits (length and colour score of focal males, and the difference in the body length and colour score of the males in the paired stimulus shoals) on the time focal males spent associating with the more attractive stimulus shoal. The $P$ value in boldface indicates a significant treatment effect on the response variable.

\begin{tabular}{|c|c|c|c|c|c|}
\hline \multicolumn{6}{|c|}{$\begin{array}{l}\text { Model: Im (asin(TimeMA) (as.factor(Population) * as.factor(Treatment) )+ } \\
\text { DiffLength +DiffColour +FocalLength +FocalColour) }\end{array}$} \\
\hline & DF & $\begin{array}{l}\text { Sum Sq } \\
\text { (Type I) }\end{array}$ & Mean Sq & F value & $\operatorname{Pr}(>\mathrm{F})$ \\
\hline Population & 2 & 0.245 & 0.123 & 1.387 & 0.253 \\
\hline Treatment (Female Presence/Absence) & 1 & 1.242 & 1.242 & 14.050 & $<0.001$ \\
\hline DiffLength & 1 & 0.066 & 0.066 & 0.746 & 0.389 \\
\hline DiffColour & 1 & 0.044 & 0.044 & 0.498 & 0.482 \\
\hline FocalLength & 1 & $<0.001$ & $<0.001$ & 0.004 & 0.950 \\
\hline FocalColour & 1 & $<0.001$ & $<0.001$ & 0.005 & 0.940 \\
\hline Population : Treatment & 2 & 0.177 & 0.088 & 1.000 & 0.370 \\
\hline Residuals & 161 & 14.230 & 0.088 & & \\
\hline
\end{tabular}

\begin{tabular}{|l|c|c|c|c|}
\hline & Estimate & Standard Error & $\mathbf{t}$ value & $\operatorname{Pr}(>|\mathbf{t}|)$ \\
\hline Intercept & 0.595 & 0.497 & 1.198 & 0.233 \\
\hline Population (Arima) & -0.121 & 0.082 & -1.469 & 0.144 \\
\hline Population (Tunapuna) & -0.161 & 0.078 & -2.056 & 0.041 \\
\hline Treatment (Female Absent) & 0.077 & 0.081 & 0.945 & 0.346 \\
\hline DiffLength & 0.012 & 0.013 & 0.904 & 0.368 \\
\hline DiffColour & 0.570 & 0.747 & 0.764 & 0.446 \\
\hline FocalLength & $-<0.001$ & 0.021 & -0.018 & 0.986 \\
\hline FocalColour & 0.108 & 1.002 & 0.108 & 0.914 \\
\hline $\begin{array}{l}\text { Population (Arima) : } \\
\text { Treatment (Female Absent) }\end{array}$ & 0.150 & 0.113 & 1.326 & 0.187 \\
\hline $\begin{array}{l}\text { Population (Tunapuna) : } \\
\text { Treatment (Female Absent) }\end{array}$ & 0.124 & 0.112 & 1.108 & 0.269 \\
\hline
\end{tabular}




\subsection{Discussion}

This study was the first to consider male-male social associations in guppies using multiple source populations in both the presence and absence of females (following on from the work of Gasparini et al. 2013 and Auge et al. 2016). When females were present in the stimulus shoals, focal males from all populations did not exhibit a preference to associate with either stimulus shoal, regardless of the phenotype of the males present in the shoals (Table 4.5, Figure 4.3). This is contrary to my a priori prediction and social theory (Bateson \& Healey 2005); I expected that males would prefer to shoal with less attractive male conspecifics in the presence of females so as to appear more sexually attractive by comparison and thereby enhance their chance of mating, as seen in the male house finch in the wild (Oh \& Badyaev 2010) and in an earlier laboratory study using the guppy (Gasparini et al. 2013). As stimulus males and females were separated from one another by a clear Plexiglas partition in the end compartments in the current experiment, it is possible that the focal males could observe that the stimulus males were unable to successfully inseminate the females and consequently may not have considered them to be significant sexual competitors.

When presented with male-only stimulus shoals, however, focal males from all populations (i.e. regardless of population predation risk level) tested showed a significant preference for sexually more attractive conspecific shoal mates, with no significant difference in response between populations (Table 4.5). It may be that males prefer to associate with attractive male conspecifics in these situations because receptive females 
will be drawn to such attractive males and thereby focal males might also benefit from indirectly encountering more females than otherwise. To provide insight into this proposition, I subsequently tested (in Chapter 5) whether a female's preference for more sexually attractive males is affected by the number of males in a given shoal.

However, it must be remembered that, because female guppies are only sexually receptive for a very limited period of time during their adult lives (immediately after maturation, and following the birth of their offspring), they are unlikely to spend much time searching for mates (Houde 1997). Another potential advantage of associating with attractive shoal mates may also lie in the multiple mating tactics employed by male guppies, in that less attractive males may compensate by attempting to sneak copulate females whilst their more attractive male shoal mates would be more successful in attracting and copulating with females by performing sigmoidal courtship displays (Jirotkul 2000; though see Řežucha and Reichard 2016).

It is also possible that the observed preference of male guppies for more attractive conspecific males, in the absence of nearby females, is not simply a consequence of sexual selection. Males may prefer to associate with large, colourful conspecifics if these individuals are better foragers (as evidenced by their larger body size and the level of dietary-obtained pigmentation; Endler 1980). One species with carotenoid-based coloration (carotenoid pigments being obtainable only through dietary means (Olson \& Owens 1998)) is the siskin (Carduelis spinus). It has been shown that the length of the yellow wing stripe in males of this species reflects both foraging ability (Senar \& Escobar 2002) and problem-solving when presented with a novel foraging problem (Mateos- 
Gonzalez et al. 2011). Plumage coloration has also been linked with foraging ability, and subsequently with nestling feeding rate and parental quality, in the blue tit (Cyanistes caeruleus) (García-Navas et al. 2012). A preference for grouping with more colourful, and therefore better foraging, individuals has been demonstrated in a social bird, the rock sparrow (Petronia petronia), where birds with more yellow ornamentation were more likely to lead larger foraging groups (Tóth \& Griggio 2011). Males may choose to associate with such individuals in order to increase their own access to these higher-quality food sources, and it may be that this factor is driving the guppy social associations in the absence of females observed in the current study. This may also partially explain the lack of preference observed in focal males when females were present, as focal males may alternately favour associating with shoals containing less attractive males to maximise their relative sexual attractiveness to females, or with shoals containing more attractive males in order to benefit from increased foraging information and opportunities, which results in a lack of overall preference for one type of male conspecific.

As noted above, there were no observable significant differences in the social group choices of male guppies across my three study populations (different predation levels) (Table 4.5, Figure 4.3) in the current study. This runs counter to my a priori prediction, as I had expected focal males from low-predation risk populations to exhibit a stronger preference to associate with more sexually attractive conspecific males compared with focal males from high-predation risk populations, who I expected would exhibit a weaker preference to associate with larger, more colourful male conspecifics because the latter are more conspicuous to predators and would therefore be associated 
with a greater individual risk of predation (Endler 1980; Godin \& McDonough 2003). One possible explanation for this finding is that the focal males did not actively perceive any risk of predation during the trials as they were performed in aquaria in the absence of any predator and under controlled conditions. It may well be costly to falsely perceive an immediate local risk of predation when one does not exist (Lima \& Dill 1990), and so it is possible that focal male guppies would make different partner-choice decisions if they in fact had perceived an imminent predation threat instead in the current experiment. Future work in Chapter 6 tests whether the presence of a water-borne chemical alarm cue, indicative of an immediate local threat of predation, has any effect on the shoaling decisions of male guppies from different riverine populations. 


\section{Chapter 5}

Winning friends and influencing females:

Does the number of available males alter female association preferences? 


\subsection{Abstract}

Previously, I found that, in the absence of females, focal male guppies preferred to associate with a group of sexually more attractive male conspecifics over a group of less attractive males. One proposed explanation for this choice was that, if this social context provides sexually more attractive male rivals with better future opportunities to encounter receptive females, focal males potentially could exploit such opportunities to sneak copulate with females that are distracted by the courtship displays of attractive males. To test this proposition here, I presented female guppies from two different populations in Trinidad with a choice of two males as potential associates (social partners or mates), one sexually attractive and the other less attractive (as measured by the proportion of their bodies covered with orange and black coloration). I then repeated the presentation, either with the same choice (i.e. between the same two stimulus males) or a different choice (i.e. two shoals of three stimulus males, where each shoal contains one of the initial males, plus two additional colour-matched shoal mates), to determine whether the focal female's initial association preference would be affected by the increased membership size of the paired stimulus male shoals. I predicted that females would prefer the more attractive male initially and, based on my previous findings (Chapter 4), that this preference would be strengthened by the addition of two extra sexually attractive stimulus males in the shoal. I found that females did not exhibit an initial preference for the more attractive male, and that the increase in stimulus shoal size did not significantly alter the time spent with the more attractive shoal. These findings suggest that the males in Chapter 4 were not likely enhancing their mating chances by 
preferentially associating with a group of more attractive males, although it should be noted that the females used here were gravid and therefore less receptive to potential mates. Future experiments using virgin females may potentially yield a different outcome. 


\subsection{Introduction}

In Chapter 4 I reported that, in the absence of females, focal male guppies preferentially associated with males that were relatively more sexually attractive than themselves. One potential explanation suggested for this observation was that by associating with such sexually attractive shoal mates, focal males might be maximising their future chances of encountering and sneak copulating with females whilst the females are distracted by the courtship displays of their shoal mates. To further examine this possibility, the experiment described in this chapter tests whether an increased number of stimulus males in a shoal would affect a female's preference for a more attractive male compared to when the male is presented alone. Two key factors that may affect a female's choice to associate with a given male are mate choice and male harassment. Females are likely to associate with males that are viewed as favourable mates (e.g. Andersson 1994; Jennions \& Petrie 1997; Clutton-Brock \& McAuliffe 2009), but at the same time will seek to avoid situations where they will be subjected to costly harassment and coercive behaviour from males (Clutton-Brock \& Parker 1995). Balancing these two factors is key in the female's choice of who to associate with. Below, I shall expand on mate choice decisions, and particularly those factors which affect the preferences of female guppies (section 5.2.1), then move on to discuss male harassment, the costs associated with it and the decisions that females may make to avoid it (section 5.2.2). Finally, I shall introduce the aims and predictions of my current experiment (section 5.2.3). 


\subsubsection{Mate choice}

Female mate choice has been well studied throughout the natural world (see reviews by Andersson 1994; Jennions \& Petrie 1997; Clutton-Brock \& McAuliffe 2009). Indeed for a long time, the focus of mate-choice studies was directed almost exclusively on females whereas males were largely thought to compete with one another and attempt to copulate with any available female (Wong \& Candolin 2005) without being particularly choosy. Females in many species have strong preferences for particular males, such as in live-bearing animals where the female must carry and provision for offspring and are mainly responsible for parental care. Such female-biased reproductive effort selects for female mate choice, which could potentially confer direct and indirect fitness benefits to choosy females (e.g. Andersson 1994; Paul 2002; Kokko et al. 2003; Andersson \& Simmons 2006). More recently, the field of male mate-choice research has greatly expanded (Bonduriansky 2001; Edward \& Chapman 2011; Schlupp 2018), and the costs of sperm production and sexual competition are now better recognised (Wedell et al. 2002).

Female mating preferences for specific males can be based on a number of different male traits (Andersson 1994; Andersson \& Iwasa 1996; Jennions \& Petrie 1997), including body size, coloration, courtship displays, and nuptial gifts (Vahed 1998; Lewis et al. 2012). In many instances, multiple cues can be involved in mate choice, not just a single cue (Candolin 2003). It has been shown that female Trinidadian guppies exhibit a preference for males with a greater amount of orange and black pigmentation on their 
bodies (Houde 1987, 1997; Auld et al. 2016), and also that females in many populations prefer larger males (Houde 1997; Herdman et al. 2004; Magellan et al. 2005; Auld et al. 2016). These preferences can vary among populations (Houde 1987; Houde \& Endler 1990; Endler \& Houde 1995; Brooks 2002) and there is some evidence of rare-phenotype preferences, where females prefer males of novel appearance (Hughes et al. 1999; Dargent et al. 2019). Female guppies from high-predation populations have also been observed to reduce their mating preferences and sexual behaviour in the presence of predators, though this was not seen in guppies from a low-predation population (Godin \& Briggs 1996). As stated previously (Chapter 2, section 2.1), female guppies are generally only receptive sexually when they first reach sexual maturity (i.e. as virgins) and for a 3-4 day period following the birth of their offspring (Houde 1997); they are thus less receptive when gravid (i.e. pregnant). Males, however, will sexually pursue and attempt copulations with receptive and non-receptive females (Houde 1997). If a female does not respond to a male's courtship display and avoids him, then the male may use an alternative mating tactic known as sneak copulation or gonopodial thrust (Liley 1966; Houde 1997; Magurran 2005). During a sneak copulation attempt, a male approaches a female from behind, quickly dart forward and attempt to copulate, regardless of cooperation; this tactic gives even non-preferred males the chance to inseminate a female (Houde 1997; Matthews \& Magurran 2000). This tactic can be used by males to fertilise non-receptive females and is found to be more commonly used by males under a greater perceived predation threat, because the more conspicuous sigmoidal courtship display may attract the attention of predators and lead to an increased risk of predation (Endler 1987; Godin 1995). 
In Chapter 4, I reported that male guppies showed a preference to associate with males more sexually attractive than themselves when females were not present nearby. One possible explanation for this social preference is that larger, more colourful males may be more sexually attractive and preferred as mates by females (Houde 1987, 1997; Auld et al. 2016), and that by associating with this type of male conspecific, males might be more likely to come into contact with receptive females than otherwise. Whilst the large, colourful males are courting the females, their less attractive associates may be able to attempt to perform sneak copulations. It may also be that by forming part of a larger group, males are more likely to encounter females compared to when they are alone. Female preference for larger groups of potential mates has been seen in other species; for example, female black grouse (Tetrao tetrix) show a preference for leks containing more males over smaller leks (Alatalo et al. 1992).

\subsubsection{Male harassment}

Although groups of males provide females with increased mating opportunities, they can also lead to unwanted attention from males that may result in coercive sexual behaviour. Male coercive behaviour can include forced copulation, intimidation or harassment (Clutton-Brock \& Parker 1995), which can be costly to females that are trying to avoid insemination by non-preferred males. In addition, more attractive females can receive disproportional harassment if they are perceived as being more attractive (i.e. sexually receptive and/or larger), a behaviour observed in Drosophila melanogaster (Long 
et al. 2009). Previous studies have shown that female Trinidadian guppies can incur fitness costs as a result of unwanted, simultaneous courtship/harassment by multiple males (Ojanguran \& Magurran 2007). For example, male sexual harassment can lead to reduced foraging rates (Magurran \& Seghers 1994c), altered social behaviour and weaker social bonds (Darden et al. 2009), and increased intrasexual aggression (Darden \& Watts 2012) in female Trinidadian guppies.

As harassment can be costly, females have evolved a number of behavioural strategies to minimize or avoid male sexual harassment. For example, in the cockroach (Diploptera punctata), females exhibit clustering behaviours to minimise their exposure to harassment from males (Stanley et al. 2018). Likewise, female mosquitofish (Gambusia holbrooki) swim closer to one another when males are present to reduce their individual risk of harassment and increase foraging efficiency (Dadda et al. 2005). A recent comparative study using six species of poeciliid fishes (including the guppy) found that the rate at which males attempted coercive (sneak) copulation events had a significant effect on female aggregation, with a greater frequency of sneak copulation attempts leading to smaller inter-individual distances among females when males are present (Dadda 2015). Female guppies minimise male harassment by preferentially occupying the deeper areas of streams and rivers, which males avoid due to the increased risk of predation in deeper waters (Croft et al. 2006b; Darden \& Croft 2008), and by resuming movement more quickly following a predation event when shoaling with males than with females (Piyapong et al. 2010). In addition, female guppies prefer to associate with conspecific females that are more sexually attractive to males than themselves, with 
sexually unreceptive females preferring receptive females as shoaling mates to make themselves relatively less attractive to potential mates and thereby minimising unwanted sexual attention (Brask et al. 2011). Similar decisions have been observed in female mosquitofish, where harassed females will preferentially join a larger shoal containing bigger females, apparently both to dilute their individual risk of harassment and to place themselves amongst sexually more attractive female conspecifics (Agrillo et al. 2006). Male harassment can also be costly for males as it has been shown that large male guppies exhibit higher rates of mortality when kept in mixed-sex aquaria (Jerry \& Brown 2017) and with both male and female sailfin mollies (Poecilia latipinna) demonstrating a loss of body condition in situations with high levels of male harassment (Makowicz \& Schlupp 2013). The costs of male harassment may even have inter-generational effects, as female guppies subject to high levels of harassment produce smaller female offspring and male offspring with shorter gonopodia (Gasparini et al. 2012).

\subsubsection{Aims and predictions}

To gain insights into how male guppies would benefit from preferentially grouping with relatively more attractive male conspecifics, as focal males were shown to do in Chapter 4, I first offered individual focal females a choice between two stimulus males (one who was more colourful, and thus more sexually attractive, than the other) and quantified the time they spent socially associating with either stimulus male (Presentation 1). I then repeated this preference test (i.e. Presentation 2), with half of the females being 
presented with the same two stimulus males as in Presentation 1 (to test for the consistency of their initial preferences) and the other half of the females being presented with a choice between two shoals of males, with one of the shoals comprising the same colourful male as in Presentation 1 and two additional colour-matched males and the other shoal comprising the same drabber male as in Presentation 1 and two additional colour-matched males (Figure 5.1). 


\section{Presentation 1}

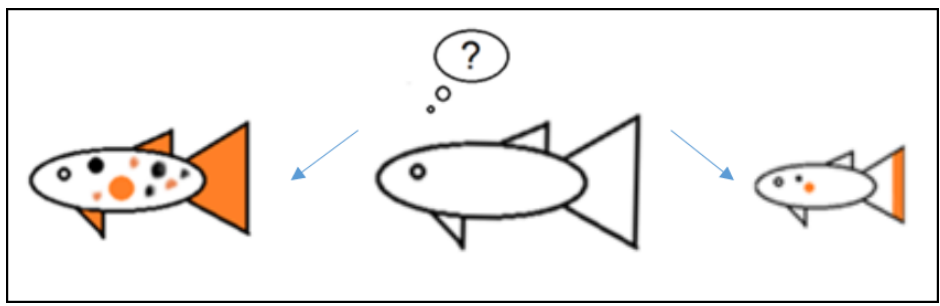

Presentation 2

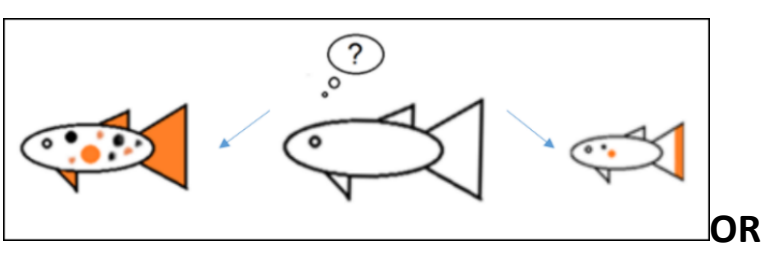

a) Treatment 1

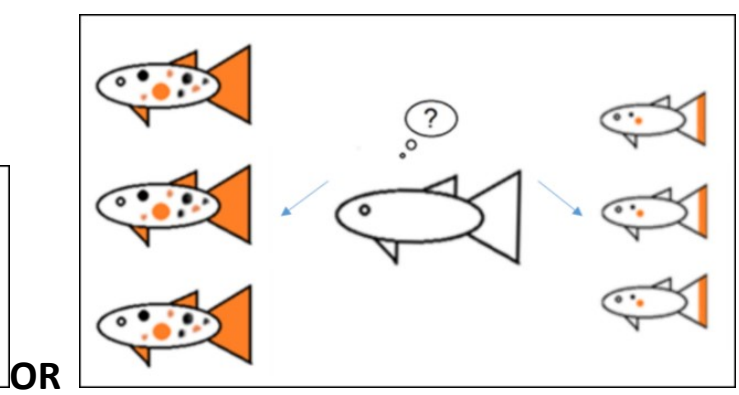

b) Treatment 2

Figure 5.1. The different stimulus males presented to individual focal female. Each female was presented with males twice. In Presentation 1, she was offered a choice between a single colourful male and a single drab male. In Presentation 2, she was assigned to one of two treatment groups: a) In Treatment 1 (the consistent stimulus group), Presentation 2 consisted of the same two males as in Presentation 1. b) In Treatment 2 (the increased stimulus group), the focal female was also presented with the same males as in Presentation 1, but in this scenario they were each accompanied by two additional males of similar phenotype, meaning that the female was now being presented with two shoals of three males each. 
The aim of this experiment was to ascertain whether increasing the number of males presented to focal females enhanced or reduced their initial preference for a more attractive male and whether any stimulus-male treatment effect on female mating preferences depended on their population of origin. The experimental guppies used originated from two populations in Trinidad differing in predation intensity. It has previously been shown that the population a female guppy comes from can affect her mating preference for certain male traits (Houde 1987; Houde \& Endler 1990; Endler \& Houde 1995; Brooks 2002). I tested three a priori predictions.

As female guppies generally prefer more colourful males over drabber ones as potential mates (Houde 1997; Auld et al. 2016), I first predicted that females in Treatment 1 (the same males for both Presentations 1 and 2) would show an initial preference for the more colourful of the paired stimulus males in Presentation 1, and that this initial preference would be maintained (i.e. be consistent over time), or even enhanced, in Presentation 2, because the focal females would already be familiar with these males and would presumably need to less time to assess them during the second presentation.

Second, in Treatment 2 (when females were presented with two individual males that differed in their sexual attractiveness in Presentation 1, and then two shoals of three males in Presentation 2), there are two alternative hypotheses/predictions regarding female association preferences. Based on my findings reported in Chapter 4, increasing the number of stimulus males presented to focal females should increase the magnitude of their expected individual preference for the initially-preferred colourful male as the number of more sexually attractive mates presented in Presentation 2 is greater than in 
Presentation 1. Alternatively, Presentation 2 should indicate a decrease in the magnitude of female preferences for the more attractive male and increase their time spent with the less attractive stimulus male because increasing the number of stimulus males increases the risk of male sexual harassment and unwanted mating attempts, which can be costly for female guppies (e.g. Magurran \& Seghers 1994c; Ojanguran \& Magurran 2007; Darden et al. 2009; Darden \& Watts 2012).

Lastly, I predicted that, on average, the magnitude of female association preferences for sexually more attractive males should be lower in females originating from a high-predation risk population compared to females originating from a lowpredation risk population, because female guppies reduce their mating preferences in general, and particularly so for sexually attractive males, when they perceive an increase in their local risk of predation (Godin \& Briggs 1996) as they are more vulnerable to predator attack when they are near visually conspicuous males (Pocklington \& Dill 1995; Segami Marzel et al. 2017).

\subsection{Methods}

\subsubsection{Experimental methods}

Guppies used in the current experiment were collected haphazardly by hand seining in two of the rivers used as sources of guppies for the previous experiment described in Chapter 4, namely, the low-predation Tunapuna River and the high- 
predation Quaré River. Collected fish were transported by car in buckets of river water to the University of the West Indies, St. Augustine and maintained in single-sex stock aquaria to prevent the development of individual social familiarity between males and females and its potential influence on the expression of female preferences. Both male and female guppies have previously been shown to demonstrate a preference for novel or unfamiliar mates (Hughes et al. 1999; Kelley et al. 1999; Dargent et al. 2019). The guppies were fed three times daily with commercial flake food, supplemented with brine shrimp nauplii. All fish were allowed to acclimatise to the laboratory for at least 48 hours prior to use in experimental trials, and only apparently healthy fish were selected for use. See Chapter 2 (section 2.2) for more details on fish husbandry.

As in previous chapters (see Chapter 2, section 2.3), the experimental apparatus consisted of a central glass test aquarium filled to $16 \mathrm{~cm}$ depth with aged tap water (Chapter 2, Figure 2.1). The bottom of the aquarium was bare. The aquarium was placed on white Styrofoam with a 10-cm wide preference zone (approximately 4 body lengths), demarcated with marker lines on the Styrofoam bottom, at each end of the aquarium. The front and back of the test aquarium were covered in brown paper to minimise potential external disturbances. The two ends that remained uncovered were each placed next to an end Plexiglas compartment, filled with the same depth of aged water as the central aquarium. The three outward-facing sides of each end compartment were also covered with brown paper to minimise disturbances to the guppies during the trial. Prior to the onset of a behavioural trial, opaque Plexiglas screens were placed between the end compartment and the central test aquarium (see Figure 2.1). Water in the aquaria was 
treated with Stresscoat ${ }^{\circledR}$ prior to each trial to minimise any stress to the fish, and water was aerated using an airstone (which was removed prior to the onset of a behavioural trial). An HD webcam (Logitech C920 HD Pro), coupled to a laptop computer, was suspended $40 \mathrm{~cm}$ above the aquarium to allow non-intrusive observations.

Each focal female was randomly assigned to one of two experimental treatment groups. Treatment 1 was a consistent stimulus group, wherein a female was presented with a choice of two stimulus males (Presentation 1), followed by a repeated choice test with the same two stimulus males (Presentation 2). Treatment 2 was an increased stimulus group, wherein a female was initially presented with a choice of two males (Presentation 2) but was then retested (Presentation 2) with a choice between two groups of 3 males each (including the two initial males from Presentation 1) (see Figure 5.1 above). Each focal female was only used in one of the two treatments and the numbers of experimental replicates are given in Table 5.1. Though females were held in single-sex aquaria prior to use, the majority would have been gravid, as they are in the wild (Houde 1997). 
Table 5.1 Number of replicates for each of the different sets of experimental conditions

\begin{tabular}{|c|c|c|}
\hline Population & Treatment 1 & Treatment 2 \\
\hline Tunapuna River (low-predation) & $\mathrm{n}=22$ & $\mathrm{n}=20$ \\
\hline Quaré River (high-predation) & $\mathrm{n}=22$ & $\mathrm{n}=17$ \\
\hline
\end{tabular}


For both treatments, the focal female was randomly selected from the female stock aquaria and placed into a clear Plexiglas cylinder $(7 \mathrm{~cm}$ diam.) in the middle of the central test aquarium. Two stimulus males were selected from the male stock aquaria. One male was selected to be more sexually attractive (MA, i.e. a greater proportion of the body area covered in orange and black pigmentation), and the other was selected as less attractive (LA or drab, less covered by black and orange pigmentation). It was initially planned that MA males would also be larger than LA males, as indicated by Figure 5.1. However, subsequent image analysis revealed that this was not always the case and, as such, sexual attractiveness here is based solely on body coloration alone. Difference in length was included as a covariate in the final statistical model (see Table 5.4).

One male was placed in each of the end compartments, with the particular compartment (right or left) assigned at random. For Treatment 2 (increased stimulus) only, a further four males ( 2 per side) were selected as additional shoal mates. Two were size- and colour-matched as closely as possible to the more attractive male, and two to the less attractive male. These fish were initially held away from the experimental aquaria and were introduced later to the experimental apparatus prior to Presentation 2.

After an initial 2-min acclimatisation period, the Presentation 1 phase was initiated by gently removing the opaque Plexiglas screens blocking the view of the focal female into the end compartments, such that the males in the end compartments were now visible to the focal female. There followed a 5-min viewing period, where the female was able to see both stimulus males whilst still constrained within the central cylinder and therefore unable to approach them. At the end of this period, the cylinder was 
removed and the female was able to move freely about the central aquarium and allowed to choose between the two stimulus males for $10 \mathrm{~min}$. I recorded the time the focal female spent in each preference zone during this period. At the end of this initial preference test, the female was gently dipnetted and returned to the central cylinder and the opaque screens were replaced so as to block her view of the males. The position of the end compartments were switched (to control for any potential side bias of the female) and then the opaque screens removed and the female allowed to view the stimulus for 5-min. Following this viewing period, a second 10-min preference test was carried out. This meant I was able to ensure that any preference exhibited by the female was for a specific male, rather than simply a particular side of the test aquarium. A female's association preference score was calculated as the amount of time she spent in the preference zone near the more attractive male(s) summed over the two 10-min preference tests (i.e. over $20 \mathrm{~min}$ ) divided by the total amount of time she spent in both preference zones, which was then expressed as a proportion to be compared against that expected by chance (proportion $=0.50)$.

At the end of the above Presentation 1 tests, the opaque screens were again replaced between the end compartments and the central test aquarium. As described in Chapter 2 (section 2.3), any female who spent more than $80 \%$ of her association time in one of the two preference zones was considered to demonstrate a side bias and was removed and discarded from further consideration. For the Treatment 2 trials, the additional stimulus males were added to the appropriate end compartments at this point. For Treatment 1 trials, no additional fish were added. The full testing procedure described 
above was then repeated for Presentation 2. Following this, all fish were removed from the apparatus, anaesthetised using dilute MS-222, placed on their side on a piece of white Plexiglas, and photographed against a metric ruler using a digital camera (Nikon Coolpix P7800). Fish body length and coloration (males only) were later measured from their individual photographs, as described in Chapter 2 (section 2.4).

\subsubsection{Statistical analyses}

All statistical analyses were carried out using the R statistical environment (R Core Team 2018, version 3.5.2). I initially performed one-sample t-tests to determine whether focal females exhibited a preference for the more attractive male(s) compared to that expected by chance (proportion $=0.50)$. I then calculated a preference difference score as the proportion of time a focal female spent associating with the more attractive male during Presentation 2 minus the proportion of time she spent associating with the more attractive male(s) during Presentation 1. A positive difference score would indicate an increase in preference for the attractive male(s) from Presentation 1 to Presentation 2, whilst a negative score would indicate a decline in the strength of the female's preference between the two presentations. I used paired t-tests to ascertain whether females showed a significant change in preference between Presentations 1 and 2.

Finally, I used a linear mixed-effects model (Proportion of time with MA Presentation Order + Population + Treatment + Length Difference + Colour Difference + (1|Female ID)) to determine the effects of population of origin, presentation order, and 
treatment (fixed factors), as well as the difference in colour and length between the stimulus shoals (covariates) on time spent with the more attractive male (see Table 5.4). I used significance testing dropping single predictors to identify the important predictors and then examined the model parameters to determine the nature of effects. I examined the model residuals and plotted Q-Q plots to confirm the normality and homogeneity of the residuals.

\subsubsection{Ethical standards}

This research was approved by the Carleton University Animal Care Committee (protocol \#104415) and adheres to the animal use and care guidelines of the Canadian Council on Animal Care and thus the laws of Canada. Wild guppies were collected from their natural home population under permit from the Fisheries Division of the Ministry of Agriculture, Land and Fisheries, Republic of Trinidad and Tobago. All healthy fish were placed in a freshwater pond on the St. Augustine campus of the University of the West Indies at the end of the experiment, with the approval of the University.

\subsection{Results}

Contrary to my a priori prediction, focal female guppies did not consistently spend significantly more time associating with the more attractive stimulus male(s) than expected by chance, irrespective of their population of origin, experimental treatment, 
and presentation number (Figure 5.2, Table 5.2). The exceptions to this were the Tunapuna River females in Treatment 1 (consistent stimulus), who exhibited a significant preference on average for the more sexually attractive male(s) in both presentations, and the Quaré River females in Treatment 2 (increased stimulus), who also exhibited a significant preference for the more sexually attractive male in Presentation 1 only (Figure 5.2 , Table 5.2).

To better visualise the effects of the different stimulus male treatments on the amount of time the females spent associating with the more attractive male(s), I calculated a difference score for each focal female in each of the two treatments and for both study populations separately, as described in the Methods above. Although the magnitude of the focal females' initial preference for the more attractive male declined (as revealed by negative difference scores) between the two consecutive presentations, none of the difference scores was statistically significant (Figure 5.3, Table 5.3). 
a) Tunapuna

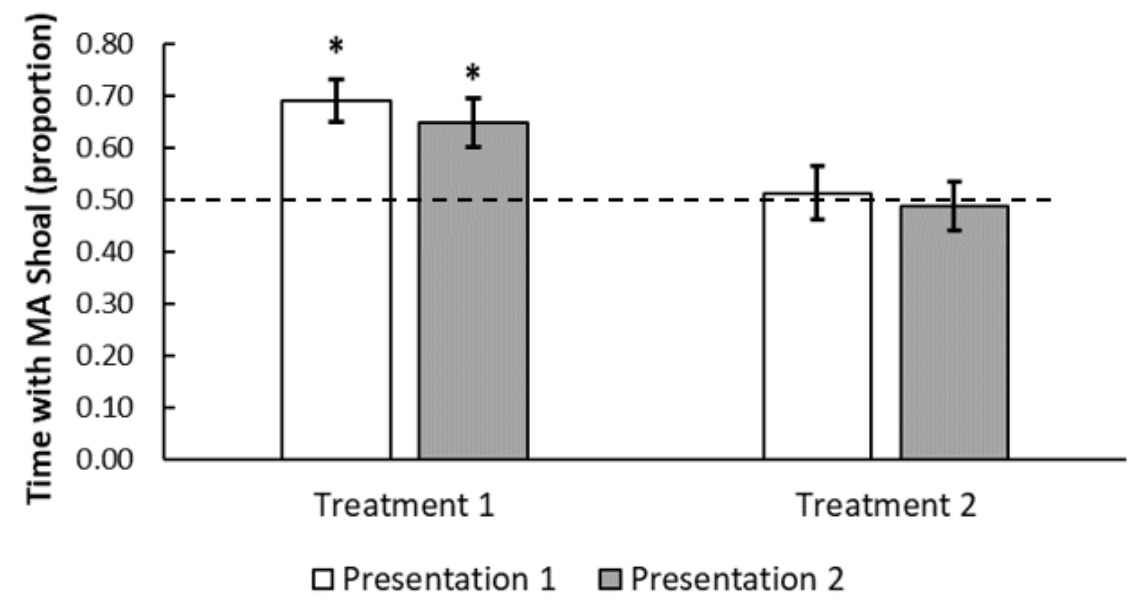

b) Quaré

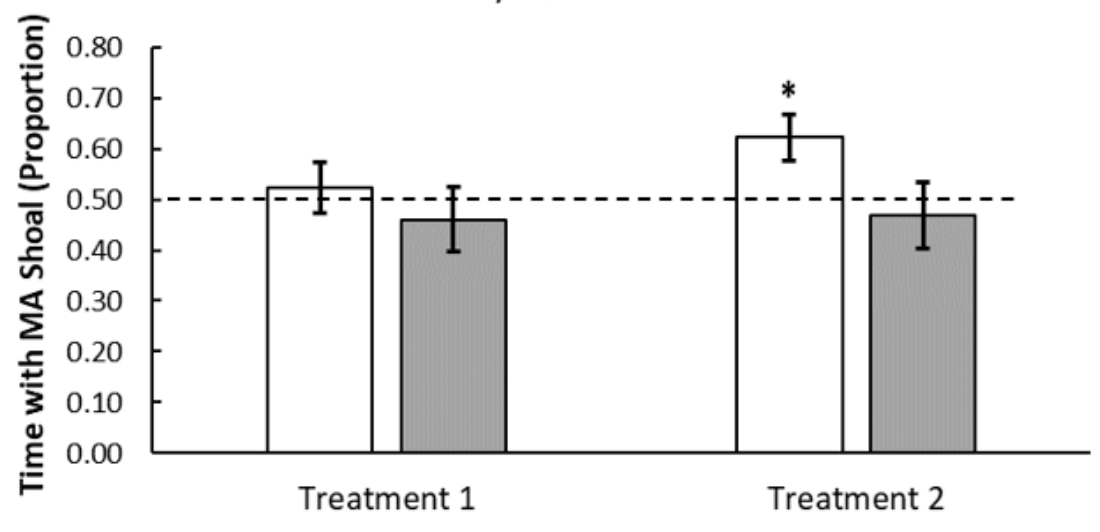

口Presentation $1 \quad \square$ Presentation 2

Figure 5.2. Mean \pm SE proportion of time spent associating with the sexually more attractive (MA) stimulus males by focal females from the a) Tunapuna River (lowpredation) and b) Quaré River (high-predation) over the two presentations in each of the two treatments. Table 5.2 below gives full results of one-sample t-tests against 0.5 (no preference). An asterisk ( $\left.{ }^{*}\right)$ above a mean denotes a choice preference for the MA shoal that is significantly greater than that expected from chance (indicated by the stippled line at 0.5). 
Table 5.2: Results of one-sample t-tests comparing the proportion of time the focal female spent near the sexually more attractive male(s) against that expected by chance (proportion $=0.50) . p$-values in boldface indicate significant differences and therefore a preference for the sexually more attractive males.

\begin{tabular}{|c|c|c|c|c|c|}
\hline Population & Treatment & Presentation & $\mathbf{t}$ & d.f. & $\mathbf{p}$ \\
\hline \multirow{4}{*}{$\begin{array}{l}\text { Tunapuna River } \\
\text { (low-predation) }\end{array}$} & \multirow[b]{2}{*}{ (Consistent stimulus) } & 1 & 4.62 & 21 & $<0.001$ \\
\hline & & 2 & 3.19 & 21 & 0.004 \\
\hline & Treatment 2 & 1 & 0.24 & 19 & 0.81 \\
\hline & (Increased stimulus) & 2 & -0.24 & 19 & 0.81 \\
\hline & Treatment 1 & 1 & 0.45 & 21 & 0.66 \\
\hline Quaré River & (Consistent stimulus) & 2 & -0.63 & 21 & 0.53 \\
\hline (mgn-preadion) & Treatment 2 & 1 & -2.69 & 16 & 0.016 \\
\hline & (Increased stimulus) & 2 & -0.48 & 16 & 0.64 \\
\hline
\end{tabular}




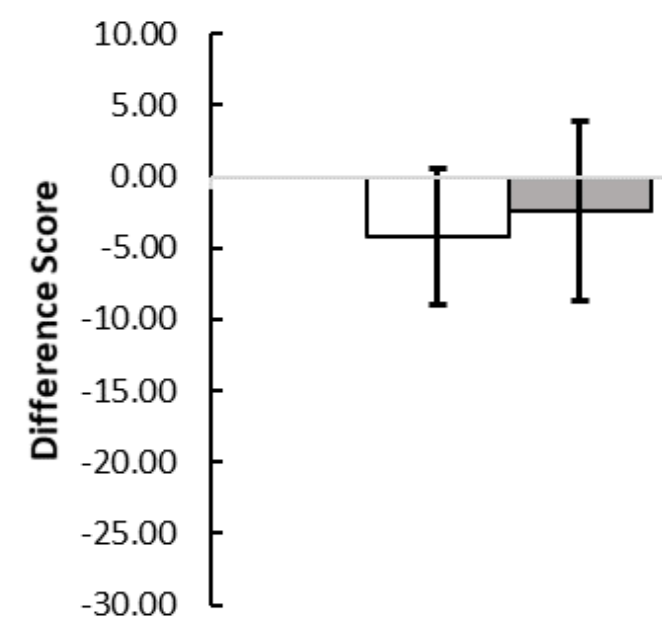

Tunapuna

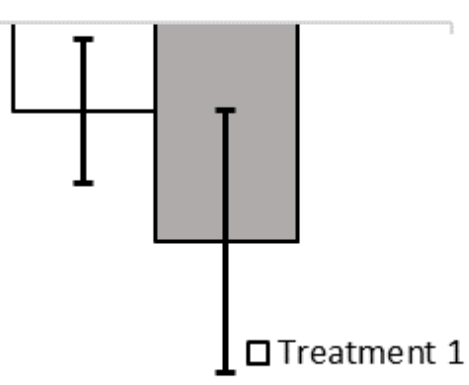

口Treatment 2

Quaré

\section{Population}

Figure 5.3: Mean \pm SE difference in preference for the more attractive shoal on the second presentation of stimulus males, either as a consistent pair of males (Treatment 1- white bars) or as part of two larger shoals each containing 3 individuals (Treatment 2-shaded bars). Negative difference scores (as seen here) indicate a decrease in preference for the more attractive shoal. Tunapuna= low-predation, Quaré= high-predation. 
Table 5.3 Results of paired t-tests comparing the proportion of time the focal female spent near the sexually more attractive male in Presentation 1 against the proportion of time spent near the sexually more attractive male(s) in Presentation 2 for each of the two treatments for both study populations. None of the p-values are statistically different.

\begin{tabular}{|c|c|c|c|c|}
\hline Population & Treatment & $\mathbf{t}$ & d.f. & P \\
\hline Tunapuna River & 1 & 0.881 & 21 & 0.388 \\
\hline (low-predation) & 2 & 0.382 & 19 & 0.707 \\
\cline { 2 - 5 } & 1 & 1.254 & 21 & 0.224 \\
\hline \multirow{2}{*}{ (high-predation) } & 2 & 1.675 & 16 & 0.113 \\
\cline { 2 - 5 } & & & & \\
\hline
\end{tabular}


I then fitted a more inclusive linear mixed-effects model considering the effects of population, presentation order, differences in phenotype between paired stimulus males (i.e. differences in both colour and length of the paired stimulus males) and treatment on the time focal females spent with the more attractive male(s). Models including the interactions between population and treatment, and treatment and presentation order were also tested, but since no interactions were significant these were excluded. Significance testing which dropped single predictors in turn revealed that presentation order, treatment and the difference in length between the paired stimulus males were all important predictors of the time focal females spent with the more attractive male(s) (Table 5.4). Examination of the model parameters demonstrates a decreased proportion of time with more attractive male(s) in Presentation 2, that there is generally a decreased proportion of time with attractive males in Treatment 2, and that as the difference in body length between more attractive and less attractive males increases, the proportion of time with more attractive male(s) increases (Table 5.5). These findings appear to be contradictory to the lack of significance found in the paired t-tests (Table 5.3), but the model is a more powerful analysis. 
Table 5.4. Results of significance testing dropping single predictors to determine important predictors in the fitted model: Proportion of time with MA Presentation Order + Population + Treatment + Length Difference + Colour Difference $+(1 \mid$ Female ID $)$. Treatment, presentation order and population were included as fixed factors. Values in boldface indicate significant effects

\begin{tabular}{|l|c|c|c|c|}
\hline & d.f & AIC & LRT & $\operatorname{Pr}$ (Chi) \\
\hline$<$ none $>$ & & -31.127 & & \\
\hline Presentation Order & 1 & -28.761 & 4.366 & $\mathbf{0 . 0 3 7}$ \\
\hline Population & 1 & -32.399 & 0.728 & 0.394 \\
\hline Treatment & 1 & -23.421 & 9.706 & $\mathbf{0 . 0 0 2}$ \\
\hline Difference in Length & 1 & -10.174 & 22.953 & $<0.001$ \\
\hline Difference in Colour & 1 & -31.114 & 2.013 & 0.156 \\
\hline
\end{tabular}


Table 5.5. The fixed effects of model parameters for the fitted model: Proportion of time with MA Presentation Order + Population + Treatment + Length Difference + Colour Difference $+(1 \mid$ Female ID)

\begin{tabular}{|l|c|c|c|}
\hline & Estimate & Standard Error & T \\
\hline Intercept & 0.544 & 0.045 & 12.013 \\
\hline Presentation Order (2) & -0.063 & 0.030 & -2.097 \\
\hline Population (Quaré) & -0.032 & 0.038 & -0.827 \\
\hline Treatment (2) & & & -3.110 \\
\hline Difference in Length & 0.032 & 0.039 & 4.933 \\
\hline Difference in Colour & 1.091 & 0.007 & 1.413 \\
\hline
\end{tabular}




\subsection{Discussion}

In the current experiment, focal female guppies exhibited a preference for associating with the more attractive male(s) in 3 of 8 treatments, with the remaining 5 treatments not exhibiting a significant preference for either shoal and associating randomly (Figure 5.2; Table 5.2). Following analysis using a linear mixed-effects model, I found that focal females spent less time on average with the more attractive male(s) in Presentation 2 compared to Presentation 1. I also found that females in Treatment 2 (increased stimulus) spent less time with the more attractive males. In addition, the amount of time that focal females spent with more attractive male(s) increased as the difference in (average) body length between the pairs of phenotypically different stimulus males increased (Table 5.5).

There are many reasons why females may choose to associate with males other than those which are considered more attractive based on their level of coloration, even though body coloration is a common male trait on which female guppies across many Trinidadian populations base their mate choice (Houde 1987, 1997; Auld et al. 2016). For example, it has previously been shown that female guppies will avoid mating with males who have recently mated with another female (Scarponi et al. 2015), a phenomenon which is seen in other species including cockroaches (Harris and Moore 2005), Drosophila (Loyau et al. 2012), and in some circumstances, crayfish (Mellan et al. 2014). Although female guppies can use chemical cues to assess a male's recent mating history (Scarponi \& Godin 2018), such cues were not available to females in this experiment as they only 
had visual cues to assess the males. It therefore seems unlikely that male prior mating history would have affected female preferences observed in the current experiment. In addition, males were held in single-sex aquaria for at least 48-hours (though generally longer) prior to experimental use. Previous studies have shown that male guppies have fully replenished sperm stores within three days after their last copulation (Pilastro et al. 2004), which should have meant that the males used in this experiment were not likely to be sperm-depleted at time of use. Finally, although female guppies have also been shown to prefer bold males regardless of their coloration (Godin \& Dugatkin 1996), focal females in the current experiment would have no way to assess how bold the stimulus males were.

One key point that should be emphasised here is that the females used in the current experiment were generally gravid and therefore less sexually receptive to males (Houde 1997), as is the case with the majority of free-ranging females in natural populations in Trinidad (Magurran 2005). This might mean that they were less interested in associating with the more attractive males and were using some other factor to make their social association decisions. As male harassment can cause significant costs to females (Ojanguran \& Magurran 2007), it would have been informative to have also observed the behaviour of the stimulus males, which I did not do here. Perhaps focal females were spending more time with the male that was less active and(or) displaying less frequently, suggesting that he was less sexually motivated and therefore less likely to attempt sneak copulations. 
One factor which did significantly affect the amount of time focal females spent associating with the more attractive stimulus male(s) was the average difference in length between the more and less attractive males. The time females spent with the more attractive male(s) tended to increase with increasing difference in body length between paired stimulus males. In many Trinidadian populations, receptive female guppies prefer larger mates on average (e.g. Houde 1997; Magellan et al. 2005; Auld et al. 2016), which may have been an important factor in the social decisions made by focal females in the current experiment. Initially, I had hoped (as in Chapters 4 and 6 ) that I would be able to use both length and colour to classify stimulus males as either less attractive or more attractive. However, whilst doing the photo analysis for this chapter it became apparent that not all of the "more attractive" males were both larger and more colourful than the "less attractive" stimulus males. The decision was taken that, as colour has more commonly been shown to correlate with sexual attractiveness to female guppies (e.g. Houde 1987, 1997; Auld et al. 2016), including in the populations from which my experimental fish originated, colour would be the measure by which attractiveness would be measured here. Difference in body length between paired stimulus males(s) was kept in the linear mixed-effects model so that it could still be considered here. Model results revealed that females were more attracted to relatively larger "more attractive" males. It thus appears that male body length was an important trait that focal females used to decide with whom to associate with, and this could either be from a mate choice perspective (i.e. Houde 1997; Magellan et al. 2005; Auld et al. 2016) or a shoaling-partner decision owing to the general preference of female guppies for size assortative shoaling 
(i.e. Croft et al. 2003b, 2005, 2009a). The larger males are still smaller than the females, but the female would appear less of an oddity in a group of large males than a group of small males.

One of the main findings of the current experiment was that females from both study populations appeared reduce their time spent associating with the more attractive male(s) during Presentation 2, and particularly so if they were assigned to Treatment 2 (i.e. were first given two individual stimulus males, and then two shoals of three males in Presentation 2), although there was no significant interaction between Treatment and Presentation number. Females were not, however, reducing the overall amount of time they spent shoaling as there was no significant difference in the proportion of time females spent within a preference zone between Presentation 1 and Presentation 2 (see Appendix 3). Female guppies are known to spend the majority of their time as part of a shoal in the wild (e.g. Griffiths \& Magurran 1998; Croft et al. 2003b), and so it appears that the focal females in this experiment were prioritising being part of a shoal over completely avoiding costly male harassment. It therefore seems possible that the focal females are demonstrating these association choices as part of a strategy to minimise harassment, whilst also remaining part of a shoal as much as possible to minimise potential risk of predation. A solitary female would be a favourable target for a predator, because adult females are typically larger than adult male guppies on average (Magurran 2005) and would therefore be a more energetically rewarding prey item.

It should be emphasised that I did not find a difference in the behavioural responses of focal females between the two study populations tested. It might have been 
expected that females from the low-predation Tunapuna River population should be more willing to remain alone (near the centre of the test aquarium and outside of the preference zones) to avoid the sexual advances of males as they are subject to less ambient predation risk in nature than females in the high risk Quaré River population. This was not seen, however, and it appears that the motivation to remain part of a shoal is similar in both of the populations tested here, regardless of their ambient predation risks in nature. An interesting future direction would be to repeat the current experiment, but with an additional female who would remain contained within a cylinder in the centre of the test aquarium. This would then allow the focal female the option to shoal without having to trade-off the costs associated with being near males.

I carried out the current experiment to better understand whether males could potentially gain a reproductive benefit by associating with groups of sexually more attractive males, as this was one of the potential explanations for the male shoaling behaviour observed in Chapter 4. However, the results of the current chapter suggest that this is not the case, at least when test females are gravid as they were here and are in the majority of cases in the wild (Houde 1997). Future work using virgin or sexually receptive females instead of gravid focal females used here could examine whether more sexually receptive females would exhibit different or stronger preferences for more sexually attractive males than observed here. 


\section{Chapter 6}

With friends like these, who needs enemies?

The effect of chemical alarm cues on male-male social

environment choice 


\subsection{Abstract}

Predation risk is a major ecological factor affecting the behaviour of prey species. Because the ambient risk of predation varies over time and space, individual animals use a variety of different predator-related cues to assess their immediate risk level and modify their behaviour accordingly to avoid predation. Here, I use a dichotomous choice paradigm to test the effects of damage-released chemical alarm cues on the shoaling preferences of male Trinidadian guppies (Poecilia reticulata) originating from two populations differing in the level of ambient fish predation. I offered focal males a choice between two maleonly stimulus shoals, one containing conspecific males that were phenotypically similar to the focal males and the other containing males phenotypically different from the focal males, in the presence of either chemical alarm cues or water as a control. I found that there was some effect of both focal male phenotype and an interaction between presence of alarm cue and population of origin on social partner preferences. In particular, whilst there was an overall preference for the more sexually attractive stimulus males as social partners in the presence of the water control (echoing the findings of Chapter 4), drab focal males from the high-predation population exhibited a tendency to shift their preference to favour social partners more phenotypically similar to themselves when chemical alarm cues were present. I also found a significant interaction between the presence or absence of alarm cues and population of origin on the shoal-switching frequency of focal males, with males from the high-predation population exposed to chemical alarm cues moving between the stimulus shoals least frequently. These results provide some evidence that the presence of chemical alarm cues leads to shifts in social 
partner preferences and movement behaviour in male guppies originating from a population that has evolved under strong fish predation pressure, though focal male phenotype seems also to have a significant effect. 


\subsection{Introduction}

Group living and assortative grouping can be important factors in reducing an individual's risk of predation (summarised in Krause \& Ruxton 2002; Ward \& Webster 2016). Predation risk can vary with both time and space (Lima \& Bednekoff 1999; Sih et al. 2000), and so prey animals often have to make use of various predation cues, including chemical cues (reviewed in Kats \& Dill 1998; Wisenden 2000; Brown 2003; Ferrari et al. 2010), in order to adjust their behaviour and make appropriate decisions, including potentially those relating to social partners.

In this chapter, I sought to examine the potential effects of damage-released chemical alarm cues on the shoaling preferences and rate of movement of male Trinidadian guppies (Poecilia reticulata). Focal males were offered a choice between two shoals, one containing males phenotypically similar to themselves, and one where the males were phenotypically different from themselves, either in the presence of a chemical alarm cue or a water control. I recorded the time that a focal male spent near either shoal as a measure of its shoaling preference and the number of times the focal male changed from associating with one stimulus shoal to the other as a measure of his rate of movement. This was done with males originating from two different populations, one characterised as high-predation risk and the other one as low-predation risk. Following on from the findings of Chapter 4, I made three a priori predictions regarding the behaviour of focal males. First, focal males should show on average a preference for the more attractive shoal (i.e. the shoal containing more sexually attractive conspecifics), 
regardless of their own personal phenotype, when tested with a water control. Second, when given the choice in the presence of chemical alarm cues, focal males should choose to associate with the shoal of conspecfic males that are phenotypically most similar to themselves (the more attractive shoal in the case of attractive focal males, and the less attractive shoal in the case of drab focal males). Third, owing to an increased individual risk of predation associated with being alone or moving (Lima \& Dill 1990; Godin 1997), focal males exposed to the chemical alarm cues should move between shoals less frequently than focal males exposed to the water control, with attractive focal males moving least of all due to their increased visual conspicuousness (Godin \& McDonough 2003; Johansson et al. 2004).

In this Introduction, I first briefly reiterate the benefits of group living and assortative shoaling (Section 6.2.1), before moving on to an overview of how predation risk can vary at the population level (Section 6.2.2). I then highlight the different types of predation cue which prey organisms can utilise to assess the risk they are exposed to (Section 6.2.3) and end the introduction with an outline of my aims and predictions for the current experiment (Section 6.2.4).

\subsubsection{Group living and assortative shoaling}

Choice of social partner can be very important in mitigating the potential risk of mortality from predation (Krause \& Ruxton 2002; Ward \& Webster 2016). In many species, individuals will preferentially choose to group with others who are phenotypically 
similar to themselves so as to reduce their risk of being attacked through the oddity effect and(or) predator confusion effect (Landeau \& Terborgh 1986; Krause \& Ruxton 2002; Ward \& Webster 2016). Where phenotype is heritable, it may also be that individuals prefer others of a similar phenotype if phenotypic familiarity predicts kinship, though studies of group relatedness do not always find this. For example, whilst there is evidence of non-random associations in the guppy, these relationships do not appear to be based on relatedness (Croft et al. 2012). Assortative shoaling can be based on many different factors, including body size (Krause et al. 1996; Hoare et al. 2000a; Croft et al. 2005; Jones et al. 2010), pigmentation or coloration (McRoberts \& Bradner 1998; Rosenthal \& Ryan 2005; Blakeslee et al. 2009), or even behavioural traits such as shoaling tendency (Croft et al. 2005). It has, however, been noted in waterfleas (Daphnia spp.) that there is a general preference for associating with larger conspecifics, as small Daphnia are at greatest risk of predation when the majority of the group is small, whilst large Daphnia are more likely to be predated when they are in the minority of a group, indicating that a phenotypic preference in grouping does not always have to be positively assortative to be adaptive (Rodgers et al. 2015).

\subsubsection{Population predation level}

As has been well-covered in previous chapters (see for example Chapter 3, section 3.2), distinct populations of the same species can commonly evolve in environments differing in predation intensity. In the Trinidadian guppy, for example, some populations 
inhabit areas where adult guppies are exposed to no predatory fish species, whilst other populations coexist with a wide array of different predatory fish species, including the pike cichlid Crenicichla alta and the blue acara cichlid Aequidens pulcher (Magurran 2005; Croft et al. 2006b; Botham et al. 2008). Individuals living in high-predation populations typically have different life histories and phenotypes, both physical and behavioural, than individuals in low-predation populations (summarised in Magurran 2005). As already discussed in Chapter 3 (section 3.2), guppies from high-predation populations are typically smaller and less colourful (Endler 1980), begin reproduction earlier (Reznick \& Endler 1982), spend less time foraging (Magurran \& Seghers 1994b) and favour less conspicuous sneak copulations over the more obvious courtship displays (Endler 1987; Magurran \& Seghers 1990, 1994b; Godin 1995) than guppies from low-predation populations. Male guppies also switch to increased sneak copulations and decreased courtship displays when predators, or some form of predation cue, are present (Magurran \& Seghers 1990; Godin 1995).

Although not observed in my earlier field observations (Chapter 3 ), there is generally an understanding that guppies in high-predation populations also spend more time shoaling with conspecifics (Seghers 1974; Magurran \& Seghers 1991, 1994a), and that these shoals are on average larger and more cohesive than those seen in lowpredation populations (Seghers 1974; Herbert-Read et al. 2017). Males from highpredation populations also exhibit less aggression towards other males than males in lowpredation populations (Magurran \& Seghers 1991). Male guppies in high-predation populations tend to spend most of their time in shallower waters (Croft et al. 2004a), as 
they are less accessible to their predators and therefore offer a level of protection. Such inter-population differences in life history, physical phenotype and behaviour are of course not exclusive to guppies. For example, in the oriental fire-bellied toad (Bombina orientalis), individuals from an island population were observed to be less brightly coloured and less active than those on the South Korean mainland, findings which appear to be linked to an increased predation risk in the island population (Kang et al. 2017).

Altered behaviours can be the result of trade-offs between minimizing individual risk of predation and maximizing other fitness-enhancing activities such as foraging and reproduction (Magurran \& Seghers 1994b). It would, however, be disadvantageous for individuals in high-predation populations to spend all their time focussed on predator avoidance, as this would likely occur to the detriment of feeding and breeding. Predators will not always be an immediate risk to an individual even though they are present in an environment. Predation risk varies in both time and space (Lima \& Bednekoff 1999; Sih et al. 2000), resulting in ecological uncertainty as individuals rarely have complete knowledge about the risks around them, and therefore seek information about their current environment to reduce this uncertainty and make appropriate behavioural responses (Dall et al. 2005; Schmidt et al. 2010). In order to account for this, individuals under high-predation pressure will often make use of a variety of predation cues to identify more immediate risk and modify their behaviour accordingly, becoming more vigilant and taking refuge or evasive action when threats appear imminent. One example can be seen in the threespot damselfish (Stegastes planifrons), which exhibit stronger avoidance behaviours when predator models approach closer to them, and also 
demonstrate heightened avoidance behaviours to larger models and those in a strike position. Whilst undertaking avoidance behaviours, damselfish also reduced feeding and territory defence behaviours to focus on avoiding an apparent imminent threat of predation (Helfman 1989).

\subsubsection{Predation cues}

Prey animals can use many different types of cues to identify the immediate risk of predation in their environment and alter their behaviour accordingly. There may be visual cues, such as the visible presence of a predator or of a conspecific giving an alarm display. They may be auditory, such as the call of a predator, the alarm calls of a conspecific or the sound of an attack. In aquatic ecosystems particularly, one of the most important types of cue are chemical cues (reviewed in Kats \& Dill 1998; Wisenden 2000; Brown 2003; Ferrari et al. 2010), either originating from the predator itself or from other prey animals as they detect a predator or are attacked and captured (reviewed in Chivers \& Smith 1998). To give a specific example, larval amphibians (two-lined salamanders, Eurycea bislineata and Cope's grey treefrog, Hyla chrysoscelis) use chemical cues to detect predatory fish and increase their time spent in refuges when these cues are present (Petranka et al. 1987). It should be noted that there are many other types of chemical cues in addition to predation cues, such as chemicals involved in mate choice (reviewed in Johannson \& Jones 2007; Thomas 2011) and species identification (Tyler et al. 2015). 
For the purpose of the current study, however, I focus solely on chemical cues related to predation.

The different types of predation cues (visual, chemical, auditory, etc.) may be considered separately or in combination and can elicit different behaviours in prey animals. For example, guppies presented with a model of a predatory cichlid were much less likely to inspect the perceived "predator" when it was paired with a chemical alarm cue, compared to when the visual stimulus was presented alongside a water control cue (Brown \& Godin 1999). Similarly, wall lizards (Podarcis muralis) will exhibit a greater antipredator response when both chemical and visual cues are present, rather than just one type alone (Amo et al. 2004). Even when just considering chemical cues, there can be differences in response depending on the exact cues present. In leopard frog tadpoles (Rana pipens), some anti-predator behaviours (such as hiding) may be induced by the presence of chemical cues indicating damaged conspecifics, but the full range of antipredator responses (behavioural and induced morphological changes) only occur when there are chemical cues that suggest a nearby predator has recently consumed and digested a conspecific (Schoeppner \& Relyea 2009).

Guppies from high-predation risk populations have also previously been shown to exhibit increased neophobic responses to a novel predator chemical cue when compared to guppies from low-predation populations, a trend which was also seen in wood frog tadpoles (Lythobates sylvaticus) (Brown et al. 2013). A subsequent study also showed that whilst both high- and low-predation guppies showed a decline in learned responses to predation cues over time, the response was retained over a longer period in those guppies 
from high-risk populations (Brown et al. 2015). Related to this, it has also been shown that guppies from high-risk populations show a greater response to visual cues of model predators than those from low-risk populations, and that high-risk guppies also react more strongly to models of predatory fish than of snakes which are not known to be a major guppy predator, indicating that these individuals are adept at discerning risk (Kelley \& Magurran 2003).

\subsubsection{Aims and predictions}

In Chapter 4, I found that male guppies from three natural populations differing in the level of predation risk showed a preference to shoal with other males that were larger and more colourful than themselves (in the absence of females). This result was counter to my initial predictions, as I expected that males would prefer less attractive shoal mates so as to appear relatively more sexually attractive to females than otherwise (as seen in male house finches, Oh \& Badyaev 2010). In addition, I did not see any differences in male shoaling behaviour between the three different populations, contrary to my initial prediction that guppies from high-predation populations should have different social preferences that would reduce their conspicuousness to predators.

It is possible that differences in shoal mate preferences were not observed among my study populations because the experiment described in Chapter 4 was performed in the laboratory, which is a predator-free environment with no immediate risk apparent to the guppies. Building on these previous results, the current experiment investigated the 
social decisions that focal male guppies make when they perceive an immediate predation risk, which was simulated here by the addition of a chemical alarm cue to the water in the testing arena prior to the onset of a shoal preference trial. The alarm cue is generated when a guppy's skin is punctured, suggesting that a predatory attack has just occurred and therefore that there is a predator in the vicinity that may pose a threat to a focal male (Brown \& Godin 1999). Here, I presented individual focal male guppies with a dichotomous choice to join either of two all-male stimulus shoals, one containing male guppies that are phenotypically similar to the focal guppy and the other containing male guppies that are phenotypically different from the focal male. A recent study using female guppies has shown that high-risk conditions, such as the presence of a chemical alarm cue, promote the formation of social networks that are strongly assorted by body size, an assortment that was not seen under low-risk conditions (Hasenjager \& Dugatkin 2017).

I predicted that male guppies from a high-predation population would show a greater preference for the shoal containing conspecific males that were most phenotypically similar to themselves when exposed to the chemical alarm cue, whilst maintaining their previously observed preference for the more attractive shoal when the water control was added to the choice arena. In contrast, I predicted that the guppies from a low-predation risk population would prefer to shoal with the relatively more attractive of the two stimulus shoals, as seen in Chapter 4, regardless of whether or not the alarm cue was present, owing to their expected reduced risk aversion (as seen in Magurran \& Seghers 1990). In addition, I also recorded the number of times focal males switched their shoaling preference completely (i.e. moved from associating with one of 
the stimulus shoals to associating with the other) as a measure of movement. I predicted that focal males exposed to the chemical alarm cue would perform fewer switches between stimulus shoals compared to males receiving the water control, because males exposed to alarm cues would be aware of potential danger and would therefore prefer to remain close to a group (i.e. switch between shoals less frequently owing to the predation cost of movement in the presence of predators; Lima \& Dill 1990). As guppies have been shown previously to prefer to shoal in high-risk situations (Seghers 1974; Magurran \& Seghers 1991, 1994a), it follows that this would be accompanied by a reduction in overall movement outside of the shoal. It should be noted that the alarm cue (or water control) is added to the choice arena only, meaning that only the focal male is exposed to it. His shoal-choice decision should therefore not be influenced by any potential social information arising from the behavioural responses of the stimulus males to the chemical cues.

\subsection{Methods}

\subsubsection{Fish collection and maintenance}

As in Chapter 5 (section 5.3), I collected my experimental guppies using seine nets from two riverine populations in Trinidad, a high-predation risk population (Quaré River) and a low-predation risk population (Tunapuna River). Collected guppies were placed in buckets containing river water and transported by car to the University of West Indies (UWI) in St. Augustine, Trinidad where they were held in population-specific, single-sex 
aquaria in an aquatics laboratory. The standard care for these guppies was as outlined in Chapter 2 (section 2.2). I used a total of 179 focal male guppies in the current experiment (87 focal fish from the Quaré River and 92 from the Tunapuna River; Table 6.1). Each focal male was only used once as a focal and therefore experienced only the chemical alarm cue or the water control, but not both. Due to numbers of fish collected, it was necessary in some cases for focal males to later be reused as stimulus males (and vice versa), but no male was ever used in more than one trial in any given day.

Focal and stimulus males were selected from different holding tanks to prevent social familiarity that may bias shoal choice (Magurran et al. 1994; Griffiths \& Magurran 1997; Croft et al. 2004c). Focal males were classified as either attractive (larger body length and more orange and black coloration) or drab (smaller and with less orange and black coloration) relative to the overall phenotype of experimental guppies collected from each population. Focal males classified as attractive were presented with a choice of one shoal of stimulus males that were closely matched in phenotype to the focal and another shoal of drab stimulus males (smaller and less colourful than the focal male). Focal males that were classified as drab were similarly presented with one shoal of stimulus males that closely resembled them phenotypically, with the other shoal containing attractive stimulus males (larger and more colourful than the focal male). This meant that each focal male experienced a choice of socially associating either with a shoal of males similar or different in phenotype to himself (Figure 6.1; Table 6.2). 
Table 6.1. Number of replicates for each of the different treatments used. Owing to inadvertent data recording errors, some of the shoal switches exhibited by the focal male in some of the trials were not noted and consequently the number of replicate trials shown below differed between the two behavioural measures.

\begin{tabular}{|c|c|c|c|c|}
\hline Population & $\begin{array}{c}\text { Focal Male } \\
\text { Type }\end{array}$ & Cue Type & $\begin{array}{c}\text { Shoaling Time } \\
\text { Replicates }\end{array}$ & $\begin{array}{c}\text { Shoal Switches } \\
\text { Replicates }\end{array}$ \\
\hline \multirow{4}{*}{$\begin{array}{c}\text { Quaré River } \\
\text { (high-predation) }\end{array}$} & \multirow{2}{*}{$\begin{array}{c}\text { More } \\
\text { Attractive }\end{array}$} & Control & $N=20$ & $\mathrm{~N}=18$ \\
\hline & & Alarm & $\mathrm{N}=21$ & $\mathrm{~N}=19$ \\
\hline & Less & Control & $\mathrm{N}=23$ & $\mathrm{~N}=23$ \\
\hline & Attractive & Alarm & $N=23$ & $N=22$ \\
\hline \multirow{4}{*}{$\begin{array}{l}\text { Tunapuna River } \\
\text { (low-predation) }\end{array}$} & \multirow{2}{*}{$\begin{array}{l}\text { More } \\
\text { Attractive }\end{array}$} & Control & $\mathrm{N}=22$ & $\mathrm{~N}=20$ \\
\hline & & Alarm & $\mathrm{N}=23$ & $N=20$ \\
\hline & \multirow{2}{*}{$\begin{array}{l}\text { Less } \\
\text { Attractive }\end{array}$} & Control & $\mathrm{N}=24$ & $\mathrm{~N}=24$ \\
\hline & & Alarm & $\mathrm{N}=23$ & $\mathrm{~N}=20$ \\
\hline
\end{tabular}


a) Colour and length phenotypes of an attractive focal male and males in the paired stimulus shoals

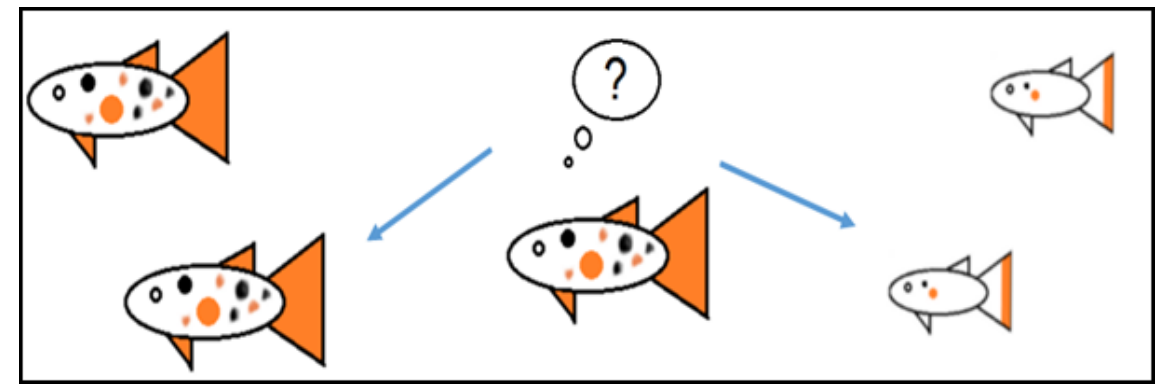

b) Colour and length phenotypes of a drab focal male and males in the paired stimulus shoals

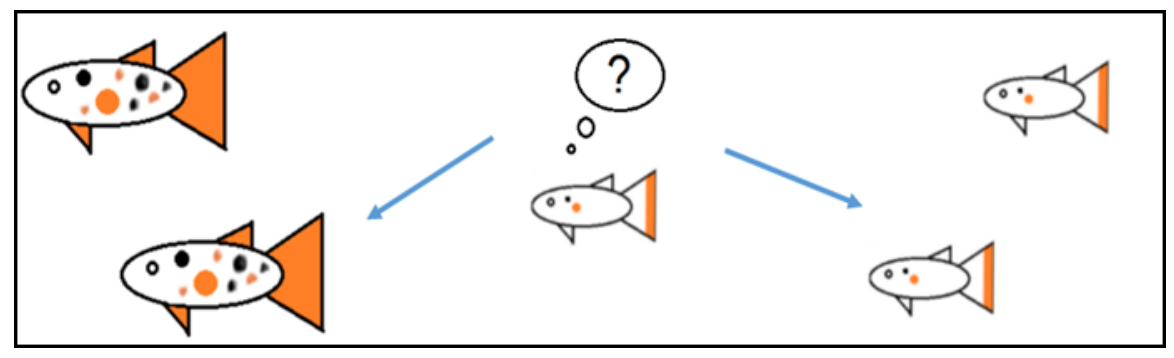

Figure 6.1. The different male phenotypes used in each of the different trials for a given population. Focal males were classified as either a) attractive or b) drab, relative to the overall population, and were always presented with a choice between a shoal phenotypically (both length and coloration) similar to himself and another shoal phenotypically different from himself. 
Table 6.2. Mean \pm SE body length $(\mathrm{mm})$ and coloration score (proportion of body covered with patches of orange and black colours) for the more attractive shoal, focal males and less attractive shoal for each of the different conditions tested.

\begin{tabular}{|c|c|c|c|c|c|c|c|c|}
\hline & & & & & & \multirow{2}{*}{\multicolumn{3}{|c|}{ Colour }} \\
\hline & & & \multicolumn{3}{|c|}{ Length } & & & \\
\hline Population & $\begin{array}{l}\text { Focal } \\
\text { Male } \\
\text { Type }\end{array}$ & $\begin{array}{l}\text { Cue } \\
\text { type }\end{array}$ & More & Focal & Less & More & Focal & Less \\
\hline \multirow{4}{*}{$\begin{array}{c}\text { Tunapuna } \\
\text { (low- } \\
\text { predation) }\end{array}$} & \multirow[t]{2}{*}{$\begin{array}{c}\text { Less } \\
\text { Attractive }\end{array}$} & Control & $\begin{array}{c}23.83 \pm \\
0.25\end{array}$ & $\begin{array}{c}21.03 \pm \\
0.30\end{array}$ & $\begin{array}{c}20.95 \pm \\
0.25\end{array}$ & $\begin{array}{c}0.094 \pm \\
0.003\end{array}$ & $\begin{array}{c}0.044 \pm \\
0.005\end{array}$ & $\begin{array}{c}0.042 \pm \\
0.003\end{array}$ \\
\hline & & Cue & $\begin{array}{c}23.96 \pm \\
0.22\end{array}$ & $\begin{array}{c}20.72 \pm \\
0.27\end{array}$ & $\begin{array}{c}21.01 \pm \\
0.25\end{array}$ & $\begin{array}{c}0.087 \pm \\
0.004\end{array}$ & $\begin{array}{c}0.051 \pm \\
0.007\end{array}$ & $\begin{array}{c}0.051 \pm \\
0.004\end{array}$ \\
\hline & \multirow[t]{2}{*}{$\begin{array}{c}\text { More } \\
\text { Attractive }\end{array}$} & Control & $\begin{array}{c}24.02 \pm \\
0.25\end{array}$ & $\begin{array}{c}24.18 \pm \\
0.17\end{array}$ & $\begin{array}{c}20.81 \pm \\
0.21\end{array}$ & $\begin{array}{c}0.090 \pm \\
0.004\end{array}$ & $\begin{array}{c}0.085 \pm \\
0.005\end{array}$ & $\begin{array}{c}0.049 \pm \\
0.005\end{array}$ \\
\hline & & Cue & $\begin{array}{c}23.75 \pm \\
0.24\end{array}$ & $\begin{array}{c}24.01 \pm \\
0.25\end{array}$ & $\begin{array}{c}20.47 \pm \\
0.22\end{array}$ & $\begin{array}{c}0.090 \pm \\
0.003\end{array}$ & $\begin{array}{c}0.088 \pm \\
0.005\end{array}$ & $\begin{array}{c}0.048 \pm \\
0.004\end{array}$ \\
\hline \multirow{4}{*}{$\begin{array}{c}\text { Quaré } \\
\text { (high- } \\
\text { predation) }\end{array}$} & \multirow[t]{2}{*}{$\begin{array}{c}\text { Less } \\
\text { Attractive }\end{array}$} & Control & $\begin{array}{c}23.70 \pm \\
0.29\end{array}$ & $\begin{array}{c}20.50 \pm \\
0.27\end{array}$ & $\begin{array}{c}20.61 \pm \\
0.30\end{array}$ & $\begin{array}{c}0.097 \pm \\
0.004\end{array}$ & $\begin{array}{c}0.050 \pm \\
0.006\end{array}$ & $\begin{array}{c}0.055 \pm \\
0.005\end{array}$ \\
\hline & & Cue & $\begin{array}{c}23.02 \pm \\
0.21\end{array}$ & $\begin{array}{c}20.30 \pm \\
0.29\end{array}$ & $\begin{array}{c}20.48 \pm \\
0.21\end{array}$ & $\begin{array}{c}0.095 \pm \\
0.003\end{array}$ & $\begin{array}{c}0.051 \pm \\
0.004\end{array}$ & $\begin{array}{c}0.056 \pm \\
0.005\end{array}$ \\
\hline & \multirow[t]{2}{*}{$\begin{array}{c}\text { More } \\
\text { Attractive }\end{array}$} & Control & $\begin{array}{c}23.29 \pm \\
0.24\end{array}$ & $\begin{array}{c}23.36 \pm \\
0.24\end{array}$ & $\begin{array}{c}20.33 \pm \\
0.23\end{array}$ & $\begin{array}{c}0.100 \pm \\
0.004\end{array}$ & $\begin{array}{c}0.095 \pm \\
0.009\end{array}$ & $\begin{array}{c}0.062 \pm \\
0.004\end{array}$ \\
\hline & & Cue & $\begin{array}{c}23.09 \pm \\
0.24\end{array}$ & $\begin{array}{c}23.13 \pm \\
0.31\end{array}$ & $\begin{array}{c}20.54 \pm \\
0.27\end{array}$ & $\begin{array}{c}0.098 \pm \\
0.004\end{array}$ & $\begin{array}{c}0.087 \pm \\
0.006\end{array}$ & $\begin{array}{c}0.059 \pm \\
0.004\end{array}$ \\
\hline
\end{tabular}


As explained above, the premise of these experiments required that focal males were offered a choice between two shoals, one of which that was phenotypically similar to himself in terms of length and coloration, and one which was different. It was also necessary that the two stimulus shoals be significantly different from each other, both in terms of length and coloration. Results of this analysis can be found in Appendix 4, confirming that in all of the different treatment combinations, the attractive and drab stimulus shoals were significantly different from one another in both length and coloration. In addition, whenever the focal male was an attractive male, the focal was significantly different in length and colour from the drab stimulus males, whilst not significantly different from the attractive stimulus shoals. In contrast, drab focal males were always significantly different from attractive stimulus shoals in both length and colour, whilst not demonstrating any significant difference in phenotype from the drab stimulus males (Appendix 4).

\subsubsection{Experimental protocol and apparatus}

The alarm cue used in this experiment was produced following the method of Brown \& Godin (1999). In brief, $0.1 \mathrm{~cm}^{2}$ of guppy skin is required to produce $1 \mathrm{ml}$ of chemical alarm cue extract, which is known to elicit antipredator behavioural responses in Trinidadian guppies (Brown \& Godin 1999). Female guppies were sacrificed as skin donors (as females are larger, this meant fewer individuals were required to obtain the required amount of skin). Donor females were killed by cervical dislocation. Their head 
and tail and internal organs were removed by dissection and discarded. The remaining body tissue (containing the skin) was used in the preparation of chemical alarm cue. Two separate batches of alarm cue were similarly produced, one using females from the Quaré River and one from the Tunapuna River. This ensured that focal males would only be exposed to conspecific alarm cues from their own population, as it has been shown that this can vary between populations, with focal guppies responding most strongly to the chemical alarm cues of conspecifics from their own population (Brown et al. 2010). Once harvested, $50 \mathrm{~cm}^{2}$ of surface area of body tissue (containing skin) was added to $200 \mathrm{ml}$ aged tap water in a beaker and then homogenised using an electric hand blender. Breaking up the skin in this matter releases chemical alarm cues, thus simulating a predation event (Brown \& Godin 1999). The homogenate was then filtered through polyester floss, and then aged tap water was added until a final volume of $500 \mathrm{ml}$ of extract was obtained. In total, 32 Quaré females and 39 Tunapuna females were sacrificed. Each population-specific alarm cue preparation was then divided into 5-ml aliquots in Nasco brand whirl packs and stored in a $-20^{\circ} \mathrm{C}$ freezer until use. Previous work has confirmed that such freezing does not subsequently reduced the alarm cues potency in eliciting antipredator behaviour in fish (Lawrence \& Smith 1989), including the Trinidadian guppy (see Brown \& Godin 1999). In control trials, aged tap water was added to the experimental aquaria instead of the chemical alarm cue. As with the alarm cue extract, this water was similarly filtered, divided into $5-\mathrm{ml}$ aliquots and stored at $-20^{\circ} \mathrm{C}$ to ensure that there was nothing in this process that could affect some trials and not others. Aliquots were thawed immediately prior to use in trials on a given day. 
The experimental apparatus used was similar to that described in Chapter 2 (section 2.3). The focal guppy was placed in a central test aquarium (arena), with the two stimulus shoals presented in separate smaller aquaria at either end (Figure 6.2). Initially, each stimulus shoal aquarium was separated from the central aquarium (choice arena) with a removable Plexiglas screen, which prevented the focal and stimulus males from seeing each other. The external sides of all aquaria were covered in brown paper to minimise any external visual disturbances to the guppies during the trials. The main difference with this set-up, compared to that outlined in Chapter 2, is that two pieces of airline tubing were affixed centrally to the front inside wall of the central aquarium. One was used to administer the added substance (chemical alarm cue, or water control), and therefore had a syringe at one end and was open to the aquarium at the other. The second line of tubing was connected to the air supply and had an airstone attached to its underwater end to lightly aerate the aquarium and to allow more effective circulation of the added substance. Prior to testing, the focal guppy was temporally placed into a clear, perforated plastic cylinder $(7 \mathrm{~cm}$ diam. $)$ in the centre of the test aquarium. The perforation of the cylinder allowed the focal guppy to be exposed to, and detect, either the alarm cue or water control, depending on the treatment, before a test trial began (Figure 6.2). 


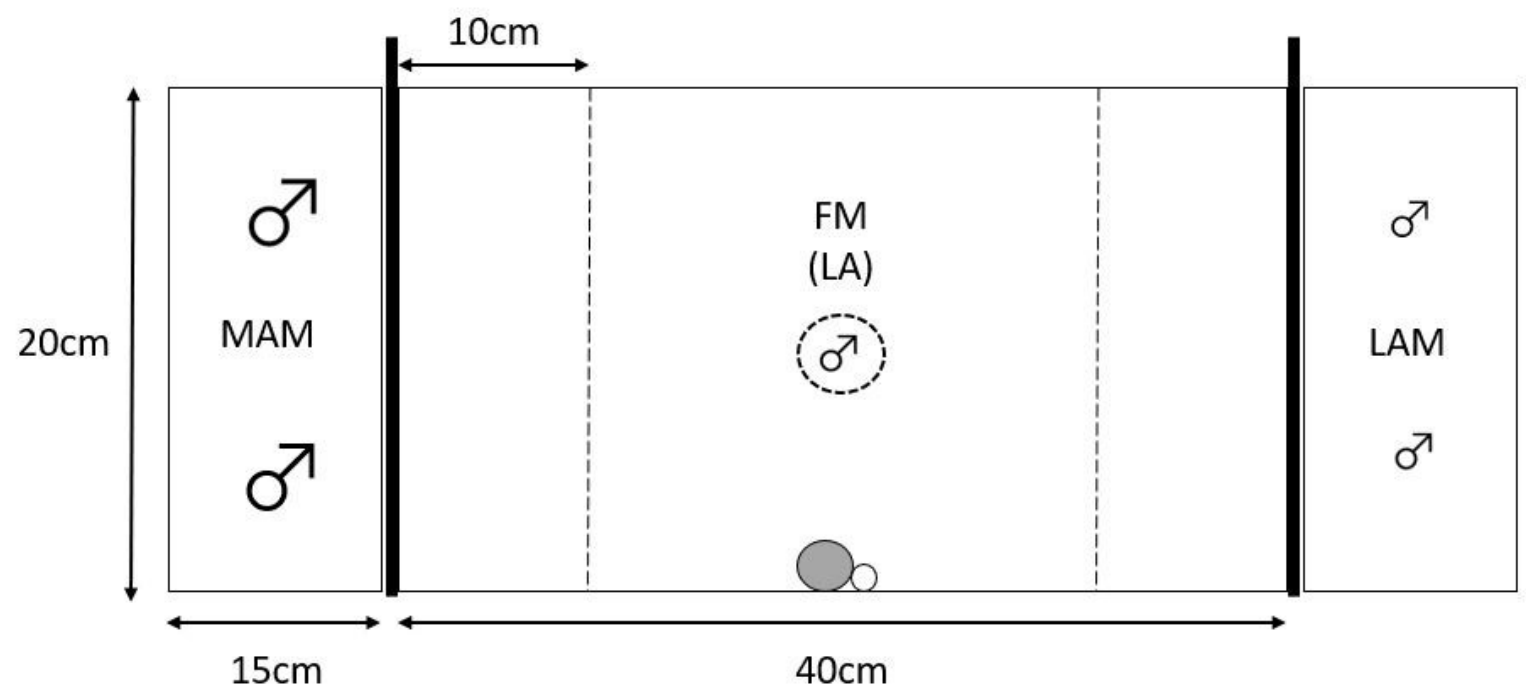

Figure 6.2. Overhead view of the experimental set-up, showing a central test arena and two smaller aquaria holding the male stimulus shoals at either end. In this diagram, a less attractive (LA) focal male (FM) is being offered a choice between a shoal of more attractive males (MAM) and a shoal of less attractive males (LAM). The central cylinder is perforated to allow exposure of the focal male to the chemical alarm cue or water cue in the central arena, depending on treatment. The open circle at the side and centre of the central arena indicates airline tubing where alarm cue or water is administered. The shaded circle is the airstone, which allows aeration of the water and distribution of the added substance. The dark thick lines denote removable opaque Plexiglas screens. The stippled lines denote a $10-\mathrm{cm}$ wide shoal preference zone in front of either stimulus shoals. 
Once the focal male was introduced into the central cylinder, it was allowed to acclimatize undisturbed for $2 \mathrm{~min}$, following which the opaque screens were removed, allowing the focal male to observe the stimulus shoals for $2 \mathrm{~min}$. Following this brief period, $2 \mathrm{ml}$ of chemical alarm cue (or water control) was injected into the central aquarium, followed $30 \mathrm{~s}$ later by an injection of $5 \mathrm{ml}$ tank water to ensure that all of the alarm cue or water control was fully flushed through the tubing and into the central test arena. The focal male was then given an additional 5 min to view the stimulus shoals whilst exposed to the injected alarm cue or water control cue, following which the cylinder was gently removed and the focal male allowed to swim freely and choose to socially associate with either of the two stimulus shoals. The behaviour of the focal male was observed using an HD-webcam (Logitech C920 HD Pro) mounted $40 \mathrm{~cm}$ above the central aquarium and coupled to a laptop computer to allow observation with minimal disturbance to the guppies. The shoal association times and shoal switches of the focal male were recorded for 10 min, after which the male was gently dipnetted and returned to the central cylinder, the opaque screens were replaced, and the positions of stimulus aquaria were swapped to control for any potential side bias of the focal male. After 2 min, the screens were removed and the focal male was allowed to view the stimulus shoals again for 5 min, following which the cylinder was again gently lifted and the focal male allowed to choose between the two stimulus shoals for a second 10-min preference test. During each of the two consecutive 10 -min preference tests, I recorded (i) the time that the focal male spent in either of the shoal preference zones and (ii) the number of times the focal male moved from the association zone of one stimulus shoal to the association 
zone of the other shoal, and back and forth, which I defined as shoal switching. Time spent near the most attractive shoal was summed over the two 10-min tests (i.e. over 20 $\min )$, and the time spent near the less attractive shoals was likewise summed over the same 20 min. Likewise, the total number of shoal switches by the focal male was summed over the 20-min trial.

At the end of the trial, all guppies were removed from the experimental aquaria, lightly anaesthetised with MS-222, placed individually on a piece of white Plexiglas and photographed next to a metric ruler using a digital camera. As detailed in Chapter 2 (section 2.4), the photographs were later analysed using ImageJ (Schneider et al. 2012) to quantify body length and body coloration for all males. Following photography, the males were placed in separate stock holding aquaria.

\subsubsection{Statistical analyses}

All statistical analyses were carried out in the R statistical environment ( $\mathrm{R}$ Core Team 2018, version 3.5.2). I began by performing a series of Wilcoxon signed-rank tests comparing the proportion of time spent with the more attractive stimulus shoal against a proportion of 0.50 (i.e. random choice) for each treatment to determine whether focal males preferred to associate with either of the paired stimulus shoals presented significantly more than expected by chance (denoted by a 0.5 proportion of total shoaling time spent with each shoal). Second, I fitted a general linear model to the data and then performed an ANOVA to test for any effect of population, focal male type and presence 
or absence of alarm cue (and the interactions between these variables) on the amount of time focal males spent shoaling with the more attractive stimulus shoal (arcsinetransformed as these data are proportional; Model: Im(asin(TimeMA) as.factor(FMType) * as.factor(Population) * as.factor(Treatment)); Table 6.4). I examined the residuals and plotted $\mathrm{Q}-\mathrm{Q}$ plots to confirm the normality and homogeneity of the residuals. I similarly fitted a general linear model to shoal switching (movement) data and performed an ANOVA to test for the effects of the same above factors on the number of shoal switches (square-root transformed to ensure normality of residuals) the focal male performed over the course of the 20-min trial (Model: Im(sqrt(Switches) as.factor(FMType) * as.factor(Population) * as.factor(Treatment)); Table 6.5).

\subsubsection{Ethical Standards}

This research was approved by the Carleton University Animal Care Committee (protocol \#104422) and adheres to the animal use and care guidelines of the Canadian Council on Animal Care and thus the laws of Canada. Wild guppies were collected from their natural home population under permit from the Fisheries Division of the Ministry of Agriculture, Land and Fisheries, Republic of Trinidad and Tobago. All healthy fish were placed in a freshwater pond on the St. Augustine campus of the University of the West Indies at the end of the experiment, with the approval of the University. 


\subsection{Results}

Using only those trials where the focal male sampled (visited) both shoal association zones during the initial 10-min preference test (so as to confirm that he was aware of the two different stimulus shoals, see Table 6.1 for replicate sample sizes), I calculated the proportion of total shoaling time that focal males spent associating with the shoal containing the more attractive stimulus males, as was done in Chapter 4, and compared it against that expected by chance (i.e. proportion $=0.50$ ) using Wilcoxon signed-rank tests (Table 6.3). I found that more attractive focal males preferentially shoaled with the more attractive stimulus shoals over the less attractive stimulus shoals (in 3 out of 4 scenarios), irrespective of treatment cue type and population of origin, except in the case of the Quaré males exposed to the alarm cue who exhibited a nonsignificant tendency to shoal preferentially with the more attractive shoal (Figure 6.3, Table 6.3). In comparison, drab focal males did not generally exhibit a preference for either stimulus shoals and thus shoaled randomly. The exception here is the drab males from the Tunapuna River that were exposed to the alarm cue, who showed a significant preference for the more attractive stimulus shoal (Figure 6.3, Table 6.3). 
a) Tunapuna

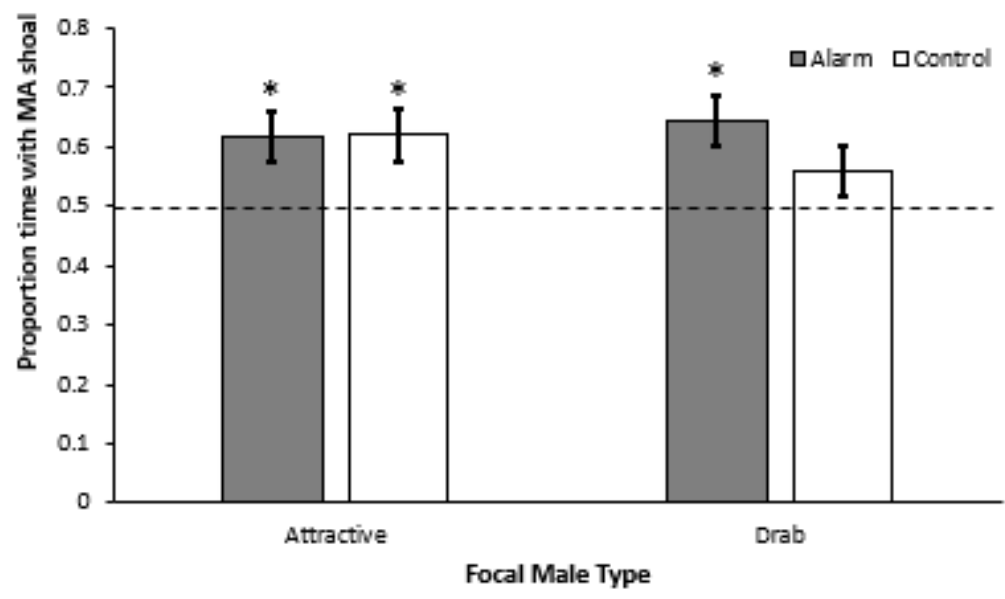

b) Quaré



Figure 6.3: Mean \pm SE proportion of total shoaling time focal males spent associating with the more attractive (MA) stimulus shoal by focal males from the (a) Tunapuna River (lowpredation) and (b) Quaré River (high-predation) in either the presence of a chemical alarm cue (shaded bars) or a water control (white bars). Table 6.4 gives results of Wilcoxon signed-rank tests comparing observed preference scores against that expected by chance (i.e. proportion $=0.50)$. An asterisk $(*)$ above a mean denotes a preference for the MA shoal that is significantly greater than expected from chance (indicated by the horizontal stippled line at 0.50). 
Table 6.3: Results of Wilcoxon signed-rank tests comparing the proportion of total shoaling time focal males spent associating with the more attractive shoal against that expected by chance (i.e. proportion $=0.50$ ). Boldface $p$ values in indicate preferences for the more attractive shoal that are greater than expected by chance.

\begin{tabular}{|c|c|c|c|c|}
\hline Population & Focal Male Type & Cue Type & $\mathbf{v}$ & $p$ \\
\hline \multirow{4}{*}{ (high-predation) } & \multirow[b]{2}{*}{ More Attractive } & Control & 173 & 0.009 \\
\hline & & Alarm & 166 & 0.082 \\
\hline & \multirow[b]{2}{*}{ Less Attractive } & Control & 170 & 0.345 \\
\hline & & Alarm & 94 & 0.190 \\
\hline \multirow[b]{2}{*}{ Tunapuna } & \multirow[b]{2}{*}{ More Attractive } & Control & 202 & 0.012 \\
\hline & & Alarm & 221 & 0.010 \\
\hline \multirow[t]{2}{*}{ (low-predation) } & \multirow[b]{2}{*}{ Less Attractive } & Control & 193 & 0.229 \\
\hline & & Alarm & 229 & 0.004 \\
\hline
\end{tabular}


When a more inclusive general linear model was fitted (Table 6.4), I found that focal male type (attractive or drab) significantly affected the shoaling decisions of focal males overall $(\mathrm{F}=4.921, \mathrm{df}=1,171, \mathrm{p}=0.029$; Table 6.4$)$. Neither cue treatment or population alone influenced the shoal choice decisions of focal males, but population and cue treatment interacted significantly $(F=4.477, d f=1,171, p=0.036$; Table 6.4).

In addition to understanding how much time focal males spent with each shoal, I also considered the rate of movement of focal males. I did this by counting the number of times in the total 20-min observation period that the focal male switched from one side of the test aquarium to the other (changing his association from one stimulus shoal to the other). I found that in general drab males performed more switches than attractive males, and that for both populations the males with the lowest number of switches were the attractive focal males exposed to alarm cue (Figure 6.4).

When the general linear model was fitted (Table 6.5), I found a significant effect of focal male type $(F=5.953, d f=1,158, p=0.016$, Table 6.5$)$ on shoal switching rate, as there was for shoaling time (Table 6.4). There was also a significant effect of population $(F=3.922, d f=1,158, p=0.049$, Table 6.5$)$ and a significant interaction between treatment (alarm cue or control) and population $(F=4.022, d f=1,158, p=0.047$, Table 6.5). 
Table 6.4. ANOVA table and parameter estimates of the general linear model testing for the effects of cue type (treatment), focal male type and population on the time focal males spent shoaling with the more attractive shoal.

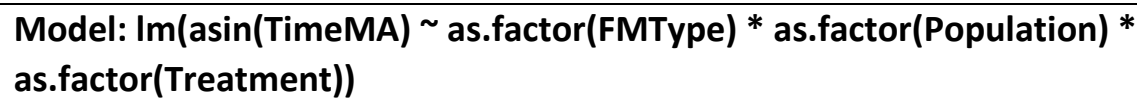

\begin{tabular}{|l|l|l|l|l|l|}
\hline & $\mathbf{d f}$ & $\begin{array}{l}\text { Sum Sq } \\
\text { (Type I) }\end{array}$ & Mean Sq & F value & Pr (>F) \\
\hline Focal Male Type (attractive or drab) & 1 & 0.529 & 0.529 & 4.921 & $\mathbf{0 . 0 2 9}$ \\
\hline Population (Tunapuna or Quaré) & 1 & 0.065 & 0.065 & 0.604 & 0.438 \\
\hline Treatment (alarm cue or control) & 1 & 0.098 & 0.098 & 0.911 & 0.341 \\
\hline Focal Male Type : Population & 1 & 0.333 & 0.333 & 3.100 & 0.080 \\
\hline Focal Male Type : Treatment & 1 & 0.016 & 0.016 & 0.147 & 0.702 \\
\hline Population: Treatment & 1 & 0.482 & 0.482 & 4.477 & $\mathbf{0 . 0 3 6}$ \\
\hline Focal Male Type : Population : Treatment & 1 & 0.099 & 0.099 & 0.920 & 0.339 \\
\hline Residuals & 171 & 18.394 & 0.108 & & \\
\hline
\end{tabular}

\begin{tabular}{|l|l|l|l|l|}
\hline & Estimate & $\begin{array}{l}\text { Standard } \\
\text { Error }\end{array}$ & t value & $\begin{array}{l}\text { Pr } \\
(>|\mathbf{t}| \mathbf{)}\end{array}$ \\
\hline Intercept & 0.644 & 0.068 & 9.411 & $<0.001$ \\
\hline Focal Male Type (Attractive) & 0.171 & 0.100 & 1.706 & 0.090 \\
\hline Population (Tunapuna) & -0.026 & 0.096 & -0.276 & 0.783 \\
\hline Treatment (Alarm Cue) & -0.179 & 0.097 & -1.850 & 0.066 \\
\hline FMT (Attractive) : Population (Tunapuna) & -0.082 & 0.139 & -0.587 & 0.558 \\
\hline FMT (Attractive) : Treatment (Alarm Cue) & 0.056 & 0.141 & 0.395 & 0.694 \\
\hline Population (Tunapuna) : Treatment (Alarm Cue) & 0.298 & 0.136 & 2.190 & 0.030 \\
\hline $\begin{array}{l}\text { FMT (Attractive) : Population (Tunapuna) : } \\
\text { Treatment (Alarm Cue) }\end{array}$ & -0.188 & 0.196 & -0.959 & 0.339 \\
\hline
\end{tabular}


a) Tunapuna

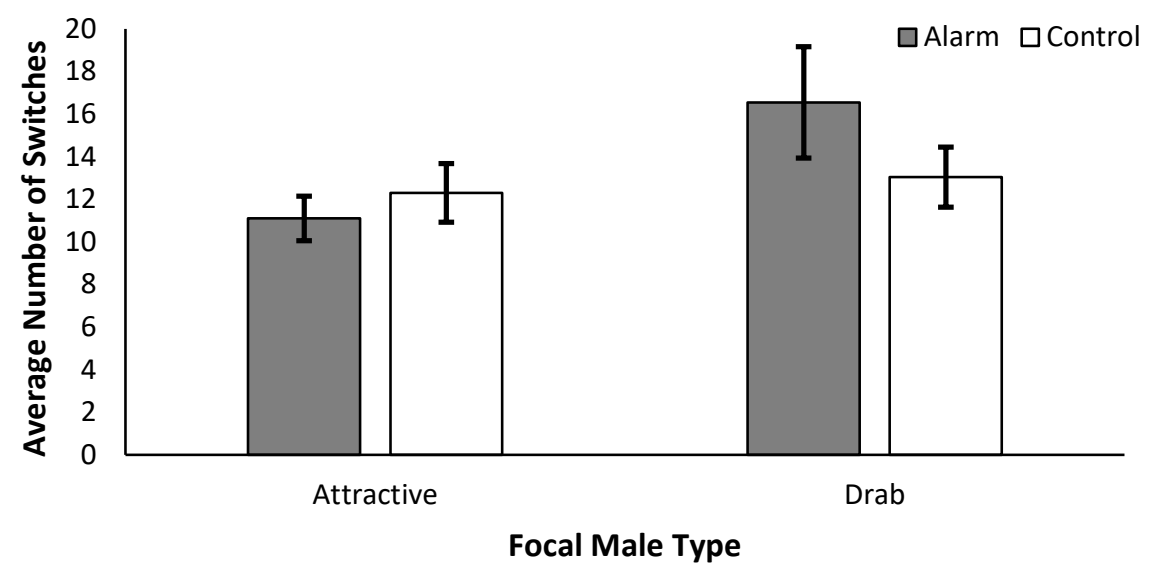

b) Quaré

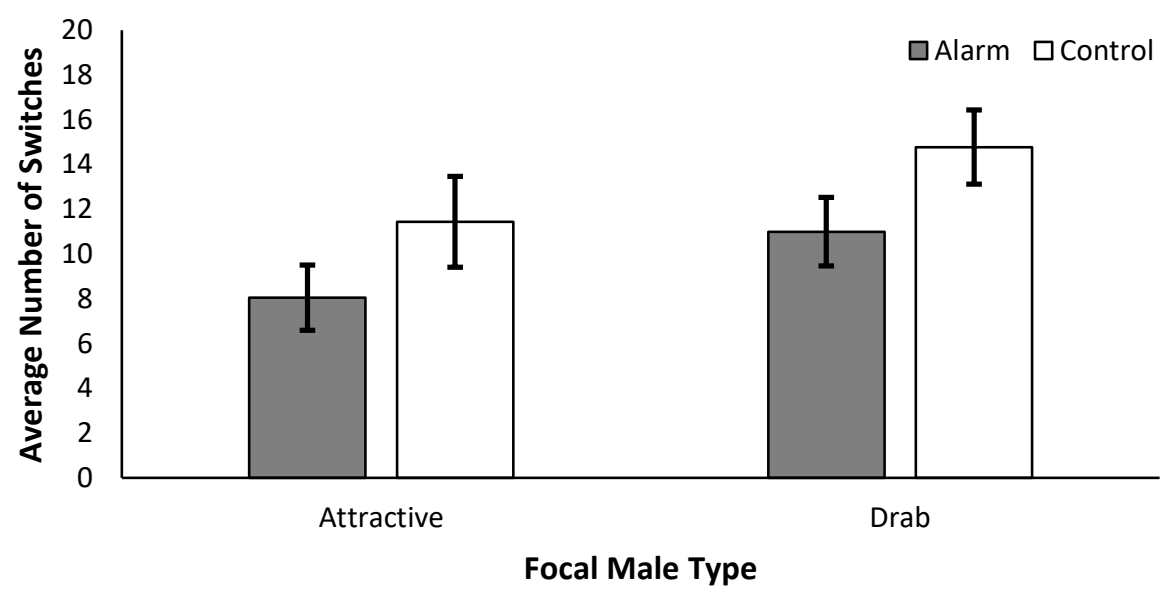

Figure 6.4. Mean $\pm \mathrm{SE}$ number of switches (movements from associating with one stimulus shoal to associating with the other shoal) in shoaling association by focal males from the (a) Tunapuna River (low-predation) and (b) Quaré River (high-predation) in either the presence of a chemical alarm cue (shaded bars) or a water control (white bars). 
Table 6.5. ANOVA table and parameter estimates of a general linear model testing for the effects of cue type, focal male type and population on the frequency of shoal switches performed by focal males.

\begin{tabular}{|l|l|l|l|l|l|}
\hline $\begin{array}{l}\text { Model: Im(sqrt(Switches) as.factor(FMType) * as.factor(Population) * } \\
\text { as.factor(Treatment)) }\end{array}$ & df & $\begin{array}{l}\text { Sum Sq } \\
\text { (Type I) }\end{array}$ & Mean Sq & $\begin{array}{l}\text { F } \\
\text { value }\end{array}$ & Pr (>F) \\
\hline & 1 & 6.881 & 6.881 & 5.953 & $\mathbf{0 . 0 1 6}$ \\
\hline Focal Male Type (attractive or drab) & 1 & 4.533 & 4.533 & 3.922 & $\mathbf{0 . 0 4 9}$ \\
\hline Population (Tunapuna or Quaré) & 1 & 2.073 & 2.073 & 1.793 & 0.182 \\
\hline Treatment (alarm cue or water control) & 1 & 0.649 & 0.649 & 0.562 & 0.455 \\
\hline Focal Male Type : Population & 1 & 0.421 & 0.421 & 0.364 & 0.547 \\
\hline Focal Male Type : Treatment & 1 & 4.649 & 4.649 & 4.022 & $\mathbf{0 . 0 4 7}$ \\
\hline Population : Treatment & 1 & 0.849 & 0.849 & 0.734 & 0.393 \\
\hline Focal Male Type : Population : Treatment & 158 & 182.630 & 1.156 & & \\
\hline Residuals & 158 & & & \\
\hline
\end{tabular}

\begin{tabular}{|l|l|l|l|l|}
\hline & Estimate & $\begin{array}{l}\text { Standard } \\
\text { Error }\end{array}$ & t value & $\begin{array}{l}\text { Pr } \\
(>|\mathbf{t}|)\end{array}$ \\
\hline Intercept & 3.711 & 0.224 & 16.552 & $<0.001$ \\
\hline Focal Male Type (Attractive) & -0.563 & 0.338 & -1.665 & 0.098 \\
\hline Population (Tunapuna) & -0.230 & 0.314 & -0.733 & 0.465 \\
\hline Treatment (Alarm Cue) & -0.593 & 0.321 & -1.848 & 0.064 \\
\hline FMT (Attractive) : Population (Tunapuna) & 0.504 & 0.470 & 1.074 & 0.285 \\
\hline FMT (Attractive) : Treatment (Alarm Cue) & 0.071 & 0.477 & 0.148 & 0.882 \\
\hline Population (Tunapuna) : Treatment (Alarm Cue) & 0.937 & 0.457 & 2.051 & 0.042 \\
\hline $\begin{array}{l}\text { FMT (Attractive) : Population (Tunapuna) : } \\
\text { Treatment (Alarm Cue) }\end{array}$ & -0.574 & 0.670 & -0.857 & 0.393 \\
\hline
\end{tabular}




\subsection{Discussion}

In the current experiment, I observed an overall significant effect of focal male type on shoaling preference, with more attractive focal males showing a stronger preference for the more attractive stimulus shoal than drabber focal males. This makes sense for two reasons; firstly, focal males should prefer to shoal assortatively when predation cues are present to minimize the oddity effect (cf. Milinski 1977; Krause \& Ruxton 2002; Ward \& Webster 2016), and secondly less attractive drab focal males might be less reproductively successful in shoals of males significantly more attractive than themselves (cf. Bateson \& Healy 2005). My previous results (Chapter 4) suggest that, in the absence of females, male guppies generally preferred to socially associate with more attractive conspecifics over less attractive ones (and this was also observed in all but one case here, though the preference was not always significant). However, in Chapter 4, all focal males were of intermediate phenotype and therefore were phenotypical "odd" compared to conspecific males in the paired stimulus shoals. In contrast, in the current experiment, focal males were offered a choice between a shoal of conspecific males that phenotypically resembled themselves and a shoal of conspecific males in which they would be noticeably odd (phenotypically different). It would thus appear adaptive for drab focal males to not associate preferentially with the more attractive shoal.

There was a significant interaction between population and alarm cue treatment, a result expected a priori. As predicted, high-predation Quaré male guppies more strongly modified their shoaling preferences in the presence of alarm cue compared with the low- 
predation Tunapuna males (Figure 6.2; Table 6.4). This is expected given that guppies in high-predation risk environments, such as the Quaré River, would have evolved under greater exposure to predator alarm cues, and therefore exhibit stronger responses to ambient predator alarm cues (Brown et al. 2013, 2015) than guppies that have evolved in low-predation risk environments, such as the Tunapuna River.

Wild Trinidadian male guppies in nature, including in the Quaré and Tunapuna Rivers, move between shoals of conspecifics very frequently (see Chapter 3, Figure 3.6; Croft et al. 2003a). Paralleling my latter field observations, focal male guppies in the current experiment also moved between the available stimulus shoals at relatively high rates on average (Figure 6.4). Shoal switching (movement) rate was significantly influenced overall by focal male type and population of origin, but not by treatment, although there was a significant river by treatment interaction (Table 6.5). These results reflect the fact that attractive focal males from the high-predation risk Quaré River that were exposed to the chemical alarm cue moved between the stimulus shoals least frequently compared with attractive males from the Tunapuna River and drab focal males from either study populations. The attractive focal males from the Quaré River are presumably more vulnerable to predation because of their larger body size and more elaborate coloration, which attract natural fish predators (Godin \& McDonough 2003; Johansson et al. 2004), compared with less attractive focal males. Additionally, prey movement increases visual conspicuousness and vulnerability to predators in general (Lima \& Dill 1990; Godin 1997). Such greater vulnerability to predation should select for lower mobility and less movement between shoals by individual Quaré River males, which 
was observed in the current experiment. Although not supported by my observations of wild guppies (Chapter 3, section 3.4.2), it is generally accepted that guppies in highpredation populations spend more time in shoals and less time alone (cf. Seghers 1974; Magurran \& Seghers 1994a), as adaptive antipredation strategies (Godin 1986; Lima \& Dill 1990).

As with shoaling preference, there was a significant interaction between treatment and population of origin affecting the shoal switching frequency of focal males in the current experiment, with high-predation Quaré focal males exhibiting fewer switches in the presence of the alarm cue when compared to the low-predation Tunapuna males. Again, given that guppies from high-predation populations, such as the Quaré River, have evolved under greater exposure to predator alarm cues, it follows that they exhibit stronger responses to ambient predator alarm cues (Brown et al. 2013, 2015), than guppies evolving in low-predation risk environments, such as the Tunapuna River which are less exposed to such cues. Attractive focal males exhibited a slight decline in their shoal switching rate on average (from 12.3 switches in the control treatment to 11.1 switches in the alarm cue treatment), whilst the drab focal males conversely exhibited an increase in shoal switching activity on average (13.04 switches in the control treatment to 16.55 switches in the alarm cue treatment). Taken together, these results suggest that adult Tunapuna River guppies have not evolved strong antipredator behavioural responses, because they have not experienced high levels of predation from piscivorous fishes (and resultant predator alarm cues) in their natural environment over evolutionary time, compared to adult guppies native to the high-predation Quaré River guppies. 
It should be emphasised that, whilst many of the findings reported in this chapter are suggestive, there are still questions about how much predation risk, as assessed by chemical alarm cues, affects social environment choice in male guppies. Focal fish were exposed only to chemical alarm cues versus a water control in the current experiment. Whilst the chemical alarm cue used here may have increased vigilance to some degree in focal males, it was not the multi-sensory cues that guppies would be exposed to in the wild when a predation threat is imminent. It has been shown previously that guppies exhibit stronger anti-predator responses when multiple types of predation cue are present rather than just one (Brown \& Godin 1999). In addition, given that the chemical alarm cue was added solely to the central test arena and influenced only the focal male, the stimulus shoals were not exposed to the alarm cue and thus their behaviour would not have been directly influenced by it. Social information can be valuable in behavioural decision making (Brown \& Laland 2003; Danchin et al. 2004; Dall et al. 2005; King \& Cowlishaw 2007; Valone 2007), and the lack of alarm/vigilance behaviour in the stimulus shoals may have provided focal males with social information that the apparent threat (suggested by the presence of chemical alarm cue) was not as imminent as perhaps they might have initially assessed. Whilst personal information about an individual's current environment is valuable, the cost of maintaining vigilance behaviour over a prolonged period of time in the absence of danger may be considerable in terms of lost feeding or courtship opportunities (Lima \& Dill 1990; Lima \& Bednekoff 1999). It may be that, whilst males exposed to the alarm cue were initially more vigilant, their risk-averse behaviours gradually declined as the trial progressed and no further cues indicative of risk of 
predation arose. As mentioned in this chapter's Introduction, there have been many studies showing that anti-predator behaviour of individuals is stronger in the presence of multiple cues indicative of predation risk (e.g. Brown \& Godin 1999; Amo et al. 2004) than a single cue alone. Moreover, the potency of the chemical alarm cue may have declined over the course of the $30-\mathrm{min}$ behavioural trial. A study of damselfish Pomacentrus ambonensis showed that chemical alarm cues aged for $30 \mathrm{~min}$ did not elicit anti-predator responses in those fish exposed to them, whilst cues aged for less time induced intermediate reactions (Chivers et al. 2013).

Overall, the results of the current experiment suggest that the phenotype of male guppies has some effect on their preferences for male social partners and movement behaviour between social groups, with some evidence of interacting effects of population of origin and the presence of chemical alarm cues. However, the results reported here are far from being definitive. Future studies should consider the duration of the chemical alarm cue's persistence in the aquatic environment, in addition to exposing stimulus fish to the same alarm cues as focal test fish to determine if social information from shoal mates is taken into consideration by focal test fish in their assessment of the local risk of predation. 


\section{Chapter 7}

\section{General Discussion}


In this thesis, I have mainly investigated and compared the social partner choices of male guppies originating from four different riverine Trinidadian populations, each differing in the level of fish predation risk as well as a variety of other factors. My field and laboratory studies have provided further evidence that male guppies, just like female guppies, exhibit social preferences and behaviours which can vary somewhat depending on their ambient and perceived risk of predation. Although female guppies are the sex most commonly considered in studies of guppy sociality, my work demonstrates that males should not be overlooked in this context either. In Chapter 3, I observed focal males as they swam freely in their home rivers and recorded their social and sexual behaviours. I found that there were no significant differences in the measures of shoaling behaviour that I recorded but did note some differences in sexual behaviour, with males from the high-predation Quaré River performing more sneak copulation attempts than males from rivers with lower ambient levels of predation intensity. Such greater expression of the sneak copulation mating tactic under high predation risk has previously been observed in other high-risk contexts (Endler 1987; Magurran \& Seghers 1990, 1994b; Godin 1995).

In addition, I also took phenotypic measurements of free-ranging male guppies collected in my four study populations and found that the low-predation Upper Aripo fish were the largest, as would be expected due to the lower risk of large individuals being targeted by predators in their home river (Endler 1995). Male guppies from highpredation populations have also been shown to mature earlier, and consequently at a smaller size, due to the increased risk of mortality from predation in their environment (Reznick \& Endler 1982). Contrary to expectation, I found that it was not the high- 
predation Quaré River males but rather males from the intermediate predation-risk Arima River population that had the least amount of body coloration on average. This population difference in male body coloration is likely best explained by the increased level of suspended sediment in the water of their home river from upstream mining since World War II (Ehlman et al. 2018), which rendered the water more turbid and likely reducing the efficacy of the coloured body markings (e.g. Maan et al. 2010).

Chapter 3 revealed what male guppies were doing, but not who they were doing it with, or if certain traits made an individual a more desirable social partner. Chapters 46 took a laboratory approach to consider the phenotypes of preferred social partners under different conditions. Chapter 4 demonstrated that the shoaling choices of male guppies are not affected by the phenotype of conspecific males when females are present, with males apparently associating randomly. This was contrary to expectations, as I had anticipated that males would prefer to associate with the shoal containing a male less attractive than himself in order to appear comparatively more attractive to the female, and thus improve his chances of securing a mating (Oh \& Badyaev 2010; Gasparini et al. 2013). However, in the absence of females, focal males chose to preferentially associate with males that were larger and more colourful than themselves, with no significant difference observed among my study populations. Two possible reasons for this preference are that, by associating with such shoal mates, focal males may increase their chances of encountering receptive females (who they can then attempt to inseminate through sneak copulation whilst their larger and more colourful associates are courting the female), or that as size and colour intensity (particularly orange) are 
indicators of fitness and foraging ability in the guppy (Endler 1980), it may be beneficial for focal males to associate with these males as they are likely adept foragers and may lead focal males to high-quality food sources.

Following on from this result, I considered whether the number of males in a shoal would affect a female's preference for that shoal, as this was one suggested explanation for the male social preference seen in Chapter 4. In Chapter 5, I found that female focal guppies did not appear to prefer to associate with more colourful males, and that increasing the shoal size did not affect this. The females used in the latter experiment were gravid and therefore not sexually receptive, which likely affected their decision, but which is also representative of the majority of wild female guppies in nature (Houde 1997; Magurran 2005). It is well-established that female guppies have a strong preference to associate with other females and tend to move and shoal to avoid male sexual harassment (Croft et al. 2006b; Darden \& Croft 2008; Piyapong et al. 2010), and so the situation they were presented with here would not be optimal as they were only able to associate with males or remain alone. However, as Appendix 3 shows, females did not reduce the amount of time that they were shoaling between the two presentations of stimulus male shoals, indicating that females are willing to accept the risk of harassment in order to remain part of a shoal, instead of swimming alone and potentially placing themselves at greater risk.

Finally, as the previous chapters show little evidence of difference in social partner choice in guppies originating from different natural populations, in Chapter 6 I manipulated the perceived risk of predation using chemical alarm cues to test whether 
males would alter their previously observed preference for more attractive shoal mates if they perceived an immediate risk of predation. I did find that overall the preference for more attractive shoal mates was maintained. However, males from the high-predation Quaré River moved between shoals significantly less when the alarm cue was present, suggesting that they were modifying their mobility so as to minimise their visual conspicuousness. This suggests that chemical cues alone are not sufficient to significantly alter a male's social partner preference, though it would be prudent to consider this in combination with other predation cues (such as visual cues) to ascertain whether individual social preferences can be altered.

Whilst the findings reported in my thesis have expanded our knowledge of the social association decisions of male guppies, the wider environmental context should also not be overlooked. Anthropogenic changes to the environment are becoming increasingly prevalent, with human induced rapid environmental changes (HIREC) being a topic of great concern (as reviewed in Palumbi 2001; Sih et al. 2011; Sih 2013). The guppy, despite its ubiquity and tenacity, is not immune from these changes. In Chapter 3, there was the suggestion that water turbidity linked to upstream mining could result in phenotypic changes (as less colourful males were found at my Arima River site), and other researchers have also suggested a number of guppy behaviours may also be affected by this, including shoaling preferences (Borner et al. 2015; Kimbell \& Morrell 2015, 2016), mating strategies (Ehlman et al. 2018) and predator avoidance (Borner et al. 2015; Kimbell \& Morrell 2015). Other types of changes can also be consequential for guppies. Chapter 6 showed the importance of chemical alarm cues in making decisions to avoid predations, particularly 
for guppies in high predation-risk populations. Studies on the rainbow trout Onchorhynchus mykiss (Brown et al. 2012) and Atlantic salmon Salmo salar (Elvidge \& Brown 2016) have shown that increased environmental acidification can lead to fish being less able to detect these alarm cues, and consequently suffer increased risks of predation. Juvenile damselfish Pomacentrus amboinensis also show ineffective learning of predation cues under acidification and fail to respond to such cues (Ferrari et al. 2012).

As Trinidad becomes increasingly developed and more roads are built, many rivers are becoming increasingly accessible which, whilst useful for researchers, means that they are subjected to greater levels of human activity, leading to increasing levels of disturbance, litter and other pollution. Studies of guppies have shown that courtship and other behaviours can be altered/affected when water becomes contaminated with agricultural chemicals such as herbicides (Shenoy 2012) and endocrine-disrupting chemicals resulting from the metabolism of growth hormones (Bertram et al. 2015), and also when the water system becomes contaminated by pharmaceuticals (e.g. Saaristo et al. 2017). My sites at the Tunapuna and Upper Aripo Rivers are clearly already disturbed by human use, and the lower Arima River is very disturbed by upstream mining activities, so the work presented in this thesis is not the result of working with fish from pristine environments. As human activity in and around the rivers in Trinidad further increases, it is likely that the guppy behaviours that I have observed for this thesis may be further altered in the future. Guppies are generally found throughout the waterways of Trinidad and appear to be able to live and reproduce in even the most disturbed waters, but this will also lead to increased stress and different trade-offs (Magurran 2005). 
In addition to the challenges and limitations posed by current and future environmental changes, it should also be remembered that, for the majority of my projects, I have only made use of guppies from one riverine population experiencing each of the predation levels considered. It is therefore necessary to consider that differences seen (or not seen) across the different populations may be due to other factors that vary between the locations (light intensity, food availability etc.) as well as, or instead of, the different predation risks that guppies encounter. A more comprehensive study comparing multiple replicate populations under different predation levels would be invaluable in further explaining the effects of population predation risk upon male social behaviour when compared to other factors.

Overall, I have demonstrated that male guppies do express phenotype-dependent social environment choices under some circumstances (particularly in the absence of females), although I cannot fully explain why this may be. Because groups of large, colourful males were not preferred by (gravid) females here, it is unlikely the aforementioned male social associations confer reproductive benefits to males. I did find that a chemical predation cue affected the rate of movement in male guppies from highpredation populations (with these males moving less when exposed to the chemical predation cue) but did not significantly alter their social partner choices. I have, however, shown that male guppies do exhibit social preferences even despite their increased fission-fusion movement when compared to female guppies.

Although female guppies are clearly the more social sex (in terms of shoaling) and their social association behaviour has been studied more extensively than that of males 
in the past, there remains much scope for further investigations of male sociality in this species and in animals in general. In particular, further experiments that seek to understand why relatively more attractive males appear to be the favoured shoal mates of focal males would potentially be enlightening. This could include repeating the experiment in Chapter 5 using virgin (and therefore receptive) females to determine whether these females are more likely to associate with larger groups of attractive males. The effect of predation cues could also be further investigated, including through exposing both focal and stimulus males to the chemical predation cue, and by presenting multiple types of cue (e.g. chemical cues accompanied by visual cues). Finally, I would suggest a series of experiments tracking the movement of individually tagged males freeswimming in a pool, to consider potential phenotypic assortment when males have a number of choices and are able to freely interact with one another (again in the presence and absence of females). 


\section{Bibliography}


Abrahams, M.V. 1993. The trade-off between foraging and courting in male guppies. Animal Behaviour, 45: 673-681.

Agrillo, C., Dadda, M. and Bisazza, A. 2006. Sexual harassment influences group choice in female mosquitofish. Ethology, 112: 592-598.

Alatalo, R.V., Höglund, J., Lundberg, A. and Sutherland, W.J. 1992. Evolution of black grouse leks : female preferences benefit males in larger leks. Behavioral Ecology, 3: 5359.

Alonzo, S.H. 2008. Female mate choice copying affects sexual selection in wild populations of the ocellated wrasse. Animal Behaviour, 75: 1715-1723.

Amo, L., López, P. and Martín, J. 2004. Wall lizards combine chemical and visual cues of ambush snake predators to avoid overestimating risk in refuges. Animal Behaviour, 67: 647-653.

Andersson, M. 1994. Sexual Selection. Princeton University Press, Princeton.

Andersson, M. and Iwasa, Y. 1996. Sexual selection. Trends in Ecology and Evolution, 11: 53-58.

Andersson, M. and Simmons, L.W. 2006. Sexual selection and mate choice. Trends in Ecology and Evolution, 21: 296-302.

Aplin, L.M., Farine, D.R., Morand-Ferron, J., Cole, E.F., Cockburn, A. and Sheldon, B.C. 2013. Individual personalities predict social behaviour in wild networks of great tits (Parus major). Ecology Letters, 16: 1365- 1372. 
Aplin, L.M., Farine, D.R., Morand-Ferron, J. and Sheldon, B.C. 2012. Social networks predict patch discovery in a wild population of songbirds. Proceedings of the Royal Society B, 279: 4199-4205.

Archie, E.A., Moss, C.J. and Alberts, S.C. 2006. The ties that bind: genetic relatedness predicts the fission and fusion of social groups in wild African elephants. Proceedings of the Royal Society B, 273: 513-522.

Auge, A.-C., Auld, H.L., Sherratt, T.N. and Godin, J.-G.J. 2016. Do males form social associations based on sexual attractiveness in a fission-fusion fish society? PLOS ONE, 11: e0151243.

Auld, H.L. and Godin, J.-G.J. 2015. Sexual voyeurs and copiers: social copying and the audience effect on male mate choice in the guppy. Behavioral Ecology and Sociobiology, 69: $1795-1807$.

Auld, H.L., Jeswiet, S.B. and Godin, J.-G.J. 2015. Do male Trinidadian guppies adjust their alternative mating tactics in the presence of a rival male audience? Behavioral Ecology and Sociobiology, 69: 1191-1199.

Auld, H.L., Pusiak. R.J. and Godin, J.-G.J. 2016. Independent mating preferences for male body size and coloration in female Trinidadian guppies. Ethology, 122: 597-608.

Auld, H.L., Ramnarine, I.W. and Godin, J.-G.J. 2017. Male mate choice in the Trinidadian guppy is influenced by the phenotype of audience sexual rivals. Behavioral Ecology, 28: $362-372$. 
Aureli, F., Schaffner,C.M., Boesch, C., Bearder, S.K., Call, J., Chapman, C.A., Connor, R., Di Flore, A., Dunbar, R.I.M., Henzi, S.P., Holekamp, K., Korstjens, A.H., Layton, R., Lee, P., Lehmann, J., Manson, J.H., Ramos-Fernandez, G., Strier, K.B. and van Schaik, C.P. 2008. Fission-fusion dynamics. Current Anthropology, 49: 627-654.

Baird, T.A. 2013. Male collared lizards, Crotaphytus collaris (Sauria: Crotaphytidae), signal females by broadcasting visual displays. Biological Journal of the Linnean Society, 108: $636-646$.

Bateson, M. and Healy, S.D. 2005. Comparative evaluation and its implications for mate choice. Trends in Ecology and Evolution, 20: 659-664.

Bertram, M.G., Saaristo, M., Baumgartner, J.B., Johnstone, C.P., Allinson, M., Allinson, G. and Wong, B.B.M. 2015. Sex in troubled waters: Widespread agricultural contaminant disrupts reproductive behaviour in fish. Hormones and Behavior, 70: 85-91.

Bisazza, A. and Marin, G. 1995. Sexual selection and sexual size dimorphism in the eastern mosquitofish Gambusia holbrooki (Pisces Poeciliidae). Ethology Ecology and Evolution, 7: 169-183.

Blakeslee, C., McRobert, S.P., Brown, A.C. and Clotfelder, E.D. 2009. The effect of body coloration and group size on social partner preferences in female fighting fish (Betta splendens). Behavioural Processes, 157-161.

Bonduriansky, R. 2001. The evolution of male mate choice in insects: a synthesis of ideas and evidence. Biological Reviews, 76: 305-339. 
Borner, K.K., Krause, S., Mehner, T., Uusi-Heikkilä, S., Ramnarine, I.W. and Krause, J. 2015. Turbidity affects social dynamics in Trinidadian guppies. Behavioral Ecology and Sociobiology, 69: 645-651.

Botham, M.S., Hayward, R.K., Morrell, L.J., Croft, D.P., Ward, J.R., Ramnarine, I.W. and Krause, J. 2008. Risk-sensitive antipredator behavior in the Trinidadian guppy, Poecilia reticulata. Ecology, 89: 3174- 3185.

Botham, M.S., Kerfoot, C.J., Louca, V. and Krause, J. 2005. Predator choice in the field: grouping guppies, Poecilia reticulata, receive more attacks. Behavioral Ecology and Sociobiology, 59: 181-184.

Botham, M.S. and Krause, J. 2005. Shoals receive more attacks from the wolf-fish (Hoplias malabaricus Bloch, 1794). Ethology, 111: 881-890.

Brask, J.B., Croft, D.P, Edenbrow, M., James, R., Bleakley, B.H., Ramnarine, I.W., Heathcote, R.J.P., Tyler, C.R., Hamilton, P.B., Dabelsteen, T. and Darden, S.K. 2019. Evolution of non-kin cooperation: social assortment by cooperative phenotype in guppies. Royal Society Open Science, 6: 181493.

Brask, J.B., Croft, D.P., Thompson, K., Dabelsteen, T. and Darden, S.K. 2011. Social preferences based on sexual attractiveness: a female strategy to reduce male sexual attention. Proceedings of the Royal Society B, 279: 1748-1753.

Briggs, S.E., Godin, J.-G.J. and Dugatkin, L.A. 1996. Mate-choice copying under predation risk in the Trinidadian guppy (Poecilia reticulata). Behavioral Ecology, 7: 151-157. 
Brooks, R. 2002. Variation in female mate choice within guppy populations: population divergence, multiple ornaments and the maintenance of polymorphism. Genetica, 116: 343-358.

Brown, C. and Laland, K.N. 2003. Social learning in fishes: a review. Fish and Fisheries, 4: 280-288.

Brown, G.E. 2003. Learning about danger: chemical alarm cues and local risk assessment in prey fishes. Fish and Fisheries, 4: 227-234.

Brown, G.E., Elvidge, C.K., Ferrari, M.C.O. and Chivers, D.P. 2012. Understanding the importance of episodic acidification on fish predator-prey interactions: Does weak acidification impair predator recognition? Science of the Total Environment, 439: 62-66.

Brown, G.E., Elvidge, C.K., Macnaughton, C.J., Ramnarine, I. and Godin, J.-G.J. 2010. Crosspopulation responses to conspecific chemical alarm cues in wild Trinidadian guppies, Poecilia reticulata: evidence for local conservation of cue production. Canadian Journal of Zoology, 88: 139-147.

Brown, G.E., Elvidge, C.K., Ramnarine, I., Ferrari, M.C.O. and Chivers, D.P. 2015. Background risk and recent experience influences retention of neophobic responses to predators. Behavioral Ecology and Sociobiology, 69: 737-745.

Brown, G.E., Ferrari, M.C.O, Elvidge, C.K., Ramnarine, I. and Chivers, D.P. 2013. Phenotypically plastic neophobia: a response to variable predation risk. Proceedings of the Royal Society B, 280: 20122712. 
Brown, G.E. and Godin, J.-G.J. 1999. Chemical alarm signals in wild Trinidadian guppies (Poecilia reticulata). Canadian Journal of Zoology, 77: 562-570.

Candolin, U. 2003. The use of multiple cues in mate choice. Biological Reviews, 78: 575595.

Cattelan. S., Lucon-Xiccato, T., Pilastro, A. and Griggio, M. 2018. Familiarity mediates equitable social associations in guppies. Behavioral Ecology: doi:10.1093/beheco/ary135

Chapman, B.B., Morrell, L.J. and Krause, J. 2009. Plasticity in male courtship behaviour as a function of light intensity in guppies. Behavioral Ecology and Sociobiology, 63: 17571763.

Chivers, D.P., Dixson, D.L., White, J.R., McCormick, M.I. and Ferrari, M.C.O. 2013. Degradation of chemical alarm cues and assessment of risk throughout the day. Ecology and Evolution, 3: 3925-3934.

Chivers, D.P. and Smith, R.J.F. 1998. Chemical alarm signalling in aquatic predator-prey systems: A review and prospectus. Écoscience, 5: 338-352.

Chivers, D.P., Zhao, X., Brown, G.E., Marchant, T.A. and Ferrari, M.C.O. 2008. Predatorinduced changes in morphology of a prey fish: the effects of food level and temporal frequency of predation risk. Evolutionary Ecology, 22: 561-574.

Christal, J. and Whitehead, H. 2001. Social affiliations within sperm whale (Physeter macrocephalus) groups. Ethology, 107: 323-340. 
Chuard, P.J.C., Brown, G.E. and Grant, J.W.A. 2016. The effects of adult sex ratio on mating competition in male and female guppies (Poecilia reticulata) in two wild populations. Behavioural Processes, 129: 1-10.

Clutton-Brock, T. and McAuliffe, K. 2009. Female mate choice in mammals. Quarterly Review of Biology, 84: 3-27.

Clutton-Brock, T.H. and Parker, G.A. 1995. Sexual coercion in animal societies. Animal Behaviour, 49: 1345-1365.

Conrad, J.L., Weinersmith, J.L., Brodin, T., Saltz, J.B. and Sih, A. 2011. Behavioural syndromes in fishes: a review with implications for ecology and fisheries management. Journal of Fish Biology, 78: 395- 435.

Couzin, I.D. and Laidre, M.E. 2009. Fission - fusion populations. Current Biology, 19: 633635.

Croft, D.P., Albanese, B., Arrowsmith, B.J., Botham, M., Webster, M. and Krause, J. 2003a. Sex-biased movement in the guppy (Poecilia reticulata). Oecologia, 137: 62-68.

Croft, D.P., Arrowsmith, B.J., Bielby, J., Skinner, K., White, E., Couzin, I.D., Magurran, A.E., Ramnarine, I. and Krause, J. 2003b. Mechanisms underlying shoal composition in the Trinidadian guppy, Poecilia reticulata. Oikos, 100: 429-438.

Croft, D.P., Arrowsmith, B.J., Webster, M. and Krause, J. 2004c. Intra-sexual preferences for familiar fish in male guppies. Journal of Fish Biology, 64: 279-283. 
Croft, D.P., Botham, M.S. and Krause, J. 2004a. Is sexual segregation in the guppy, Poecilia reticulata, consistent with the predation risk hypothesis? Environmental Biology of Fishes, 71: $127-133$.

Croft, D.P., Darden, S.K. \& Ruxton, G.D. 2009a. Predation risk as a driving force for phenotypic assortment: a cross-population comparison. Proceedings of the Royal Society B, 276: 1899-904.

Croft, D.P., Hamilton, P.B., Darden, S.K., Jacoby, D.M.P., James, R., Bettaney, E.M. and Tyler, C.R. 2012. The role of relatedness in structuring the social network of a wild guppy population. Oecologia, 170: 955-963.

Croft, D.P., James, R., Thomas, P.O.R., Hathaway, C., Mawdsley, D., Laland, K.N. and Krause, J. 2006a. Social structure and co-operative interactions in a wild population of guppies (Poecilia reticulata). Behavioral Ecology and Sociobiology, 59: 644-650.

Croft, D.P., James, R., Ward, A.J.W., Botham, M.S., Mawdsley, D. and Krause, J. 2005. Assortative interactions and social networks in fish. Oecologia, 143: 211-219.

Croft, D.P., Krause, J., Darden, S.K., Ramnarine, I.W., Faria, J.J. and James, R. 2009b. Behavioural trait association in a social network: patterns and implications. Behavioral Ecology and Sociobiology, 63: 1495-1503.

Croft, D.P., Krause, J. and James, R. 2004b. Social networks in the guppy (Poecilia reticulata). Proceedings of the Royal Society B, 271: S516-S519. 
Croft, D.P., Morrell, L.J., Wade, A.S., Piyapong, C., Ioannou, C.C., Dyer, J.R.G., Chapman, B.B., Wong, Y. and Krause, J. 2006b. Predation risk as a driving force for sexual segregation: A cross-population comparison. American Naturalist, 167: 867-878.

Dadda, M. 2015. Female social response to male sexual harassment in poeciliid fish: a comparison of six species. Frontiers in Psychology, 6: 1453.

Dadda, M., Pilastro, A. and Bisazza, A. 2005. Male sexual harassment and female schooling behaviour in the eastern mosquitofish. Animal Behaviour, 70: 463-471.

Dall, S.R.X., Giraldeau, L.-A., Olsson, O., McNamara, J.M. and Stephens, D.W. 2005. Information and its use by animals in evolutionary ecology. Trends in Ecology and Evolution, 20: 187-193.

Danchin, É., Giraldeau, L.-A., Valone, T.J. and Wagner, R.H. 2004. Public information: From nosy neighbors to cultural evolution. Science, 305: 487-491.

Darden, S.K., and Croft, D.P. 2008. Male harassment drives females to alter habitat use and leads to segregation of the sexes. Biology Letters, 4: 449-451.

Darden, S.K., James, R., Ramnarine, I.W. and Croft, D.P. 2009. Social implications of the battle of the sexes: sexual harassment disrupts female sociality and social recognition. Proceedings of the Royal Society B, 276: 2651-2656.

Darden, S.K. and Watts, L. 2012. Male sexual harassment alters female social behaviour towards other females. Biology Letters, 8: 186-188. 
Dargent, F., Chen, L., Fussmann, G.F., Ghalambor, C.K. and Hendry, A.P. 2019. Female preference for novel males constrains the contemporary evolution of assortative mating in guppies. Behavioral Ecology, doi: https://doi.org/10.1093/beheco/ary202

Deacon, A.E., Farrell, A.D. and Fraser, D.F. 2015. Observation of a semi-aquatic spider attack: An overlooked fish predator in a well studied ecosystem? Living World, Journal of the Trinidad and Tobago Field Naturalists' Club, 2015: 57-59.

Deacon, A.E., Jones, F.A.M. and Magurran, A.E. 2018. Gradients in predation risk in a tropical river system. Current Zoology, 64: 213-221.

Dingemanse, N.J. and Réale, D. 2005. Natural selection and animal personality. Behaviour, 142: $1159-1184$.

Drewe, J. A. 2010. Who infects whom? Social networks and tuberculosis transmission in wild meerkats. Proceedings of the Royal Society B, 277: 633-642.

Dubois, F. and Belzile, A. 2012. Audience effect alters male mating preferences in zebra finches (Taeniopygia guttata). PLoS One, 7: e43697.

Dugatkin, L.A. and Godin, J.-G.J. 1992. Reversal of female mate choice by copying in the guppy (Poecilia reticulata). Proceedings of the Royal Society of London B, 249: 179-184.

Dussault, G.V. and Kramer, D.L. 1981. Food and feeding behavior of the guppy, Poecilia reticulata (Pisces: Poeciliidae). Canadian Journal of Zoology, 59: 684-701. 
Edenbrow, M., Darden, S.K., Ramnarine, I.W., Evans, J.P., James, R. and Croft, D.P. 2011. Environmental effects on social interaction networks and male reproductive behaviour in guppies, Poecilia reticulata. Animal Behaviour, 81: 551- 558.

Edward, D.A. and Chapman, T. 2011. The evolution and significance of male mate choice. Trends in Ecology and Evolution, 26:647-654.

Ehlman, S.M., Martinez, D., and Sih, A. 2018. Male guppies compensate for lost time when mating in turbid water. Behavioral Ecology and Sociobiology, 72: 46.

Elvidge, C.K. and Brown, G.E. 2014. Predation costs of impaired chemosensory risk assessment on acid-impacted juvenile Atlantic salmon (Salmo salar). Canadian Journal of Fisheries and Aquatic Sciences, 71: 756-762.

Endler, J.A. 1980. Natural selection on color patterns in Poecilia reticulata. Evolution, 34: 76-91.

Endler, J.A. 1983. Natural and sexual selection on color patterns in poeciliid fishes. Environmental Biology of Fishes, 9: 173-190.

Endler, J.A. 1987. Predation, light intensity and courtship behaviour in Poecilia reticulata (Pisces: Poeciliidae). Animal Behaviour, 35: 1376-1385.

Endler, J.A. 1995. Multiple-trait coevolution and environmental gradients in guppies. Trends in Ecology and Evolution, 10: 22-29.

Endler, J.A. and Houde, A.E. 1995. Geographic variation in female preferences for male traits in Poecilia reticulata. Evolution, 49: 456-468. 
Ferrari, M.C.O, Manassa, R.P., Dixon, D.L., Munday, P.L., McCormick, M.I., Meekan, M.G., Sih, A. and Chivers, D.P. 2012. Effects of ocean acidification on learning in coral reef fishes. PLos ONE, 7: e31478.

Ferrari, M.C.O., Wisenden, B.D. and Chivers, D.P. 2010. Chemical ecology of predator-prey interactions in aquatic ecosystems: a review and prospectus. Canadian Journal of Zoology, 88: 698-724.

Fortin, D., Fortin, M.-E., Beyer, H.L., Duchesne, T., Courant, S. and Dancose, K. 2009. Group-size-mediated habitat selection and group fusion-fission dynamics of bison under predation risk. Ecology, 90: 2480-2490.

Fraser, D.F., Gillam, J.F., Akkara, J.T., Albanese, B.W. and Snider, S.B. 2004. Night feeding by guppies under predator release: Effects on growth and daytime courtship. Ecology, 85: 312-319.

Galef, Jr., B.G., Lim, T.C.W. and Gilbert, G.S. 2008. Evidence of mate choice copying in Norway rats, Rattus norvegicus. Animal Behaviour, 75: 1117-1123.

Garcías-Navas, V., Ferrer, E.S. and Sanz, J.J. 2012. Plumage yellowness predicts foraging ability in the blue tit Cyanistes caeruleus. Biological Journal of the Linnean Society, 106: 418-429.

Gasparini, C., Devigili, A. and Pilastro, A. 2012. Cross-generational effects of sexual harassment on female fitness in the guppy. Evolution, 66: 532-543. 
Gasparini, C., Serena, G. and Pilastro, A. 2013. Do unattractive friends make you look better? Context-dependent male mating preferences in the guppy. Proceedings of the Royal Society B. 280: 20123072.

Godin, J.-G.J. 1986. Antipredator function of shoaling in teleost fishes: A selective review. Naturaliste Canadien (Revue Écologie \& Systématique), 113: 241-250.

Godin, J.-G.J. 1995. Predation risk and alternative mating tactics in male Trinidadian guppies (Poecilia reticulata). Oecologia, 103: 224-229.

Godin, J.-G.J. (ed.) 1997. Behavioural ecology of teleost fishes. Oxford University Press, Oxford.

Godin, J.-G.J. and Auld, H.L. 2013. Covariation and repeatability of male mating effort and mating preferences in a promiscuous fish. Ecology and Evolution, 3: 2020-2029.

Godin, J.-G.J. and Briggs, S.E. 1996. Female mate choice under predation risk in the guppy. Animal Behaviour, 51: 117-130.

Godin, J.-G.J. and Dugatkin, L.A. 1996. Female mating preference for bold males in the guppy, Poecilia reticulata. Proceedings of the National Academy of Sciences of the United States of America, 93: 10262-10267.

Godin, J.-G.J. and McDonough, H.E. 2003. Predator preference for brightly colored males in the guppy: a viability cost for a sexually selected trait. Behavioral Ecology, 14: 194-200. 
Grether, G.F., Hudon, J. and Millie, D.F. 1999. Carotenoid limitation of sexual coloration along an environmental gradient in guppies. Proceedings of the Royal Society of London B, 266: 1317-1322.

Grether, G.F., Millie, D.F., Bryant, M.J., Reznick, D.N. and Mayea, W. 2001. Rain forest canopy cover, resource availability and life history evolution in guppies. Ecology, 82: 15461559.

Griffiths, S.W. and Magurran, A.E. 1997. Familiarity in schooling fish: how long does it take to acquire? Animal Behaviour, 53: 945-949.

Griffiths, S.W. and Magurran, A.E. 1998. Sex and schooling behaviour in the Trinidadian guppy. Animal Behaviour, 56: 689-693.

Guevara-Fiore, P., Stapley, J. and Watt, P.J. 2010. Mating effort and female receptivity: How do male guppies decide when to invest in sex? Behavioral Ecology and Sociobiology, 64: $1665-1672$.

Hamilton, W.D. 1971. Geometry for the selfish herd. Journal of Theoretical Biology, 31: 295-311.

Harris, W.E. and Moore, P.J. 2005. Female mate preference and sexual conflict: Females prefer males that have had fewer consorts. American Naturalist, 165: S64-S71.

Hasenjager, M.J. and Dugatkin, L.A. 2017. Fear of predation shapes social network structure and the acquisition of foraging information in guppy shoals. Proceedings of the Royal Society B, 284: 20172020. 
Helfman, G.S. 1989. Threat-sensitive predator avoidance in damselfish-trumpetfish interactions. Behavioral Ecology and Sociobiology, 24: 47-58.

Herbert-Read, J.E., Rosén, E., Szorkovszky, A., loannou, C.C., Rogell, B., Perna, A., Ramnarine, I.W., Kotrschal, A., Kolm, N., Krause, J. and Sumpter, D.J.T. 2017. How predation shapes the social interaction rules of shoaling fish. Proceedings of the Royal Society B, 284: 20171126.

Herdman, E.J.E., Kelly, C.D. and Godin, J.-G.J. 2004. Male mate choice in the guppy (Poecilia reticulata): Do males prefer larger females as mates? Ethology, 110: 97-111.

Hoare, D.J., Krause, J., Peuhkuri, N. and Godin, J.-G.J. 2000a. Body size and shoaling in fish. Journal of Fish Biology, 57: 1351-1366.

Hoare, D.J., Ruxton, G.D., Godin, J.-G.J. and Krause, J. 2000b. The social organization of free-ranging fish shoals. Oikos, 89: 546-554.

Houde, A.E. 1987. Mate choice based upon naturally occurring color-pattern variation in a guppy population. Evolution, 41: 1-10.

Houde, A.E. 1997. Sex, color and mate choice in guppies, Princeton University Press, Princeton.

Houde, A.E. and Endler, J.A. 1990. Correlated evolution of female mating preferences and male color patterns in the guppy Poecilia reticulata. Science, 248: 1405-1408. 
Hoy, S.R., Millon, A., Petty, S.J., Whitfield, D.P. and Lambin, X. 2016. Food availability and predation risk, rather than intrinsic attributes, are the main factors shaping the reproductive decisions in a long-lived predator. Journal of Animal Ecology, 85: 892-902.

Hughes, K.A., Du, L., Rodd, H. and Reznick, D.N. 1999. Familiarity leads to female mate preference for novel males in the guppy, Poecilia reticulata. Animal Behaviour, 58: 907916.

Ioannou, C.C., Tosh, C.R., Neville, L. and Krause, J. 2008. The confusion effect - from neural networks to reduced predation risk. Behavioral Ecology, 19: 126-130.

Jennions, M.D. and Petrie, M. 1997. Variation in mate choice and mating preferences: A review of the causes and consequences. Biological Reviews, 72: 283-327.

Jerry, M. and Brown, C. 2017. Fitness costs of sexual harassment - the price of persuasion. Ethology, 123: 1-9.

Jeswiet, S.B. and J.-G.J. Godin. 2011. Validation of a method for quantifying male mating preferences in the guppy (Poecilia reticulata). Ethology, 17: 422-429.

Jeswiet, S.B., Lee-Jenkins, S.S.Y. and Godin, J.-G.J. 2012. Concurrent effects of sperm competition and female quality on male mate choice in the Trinidadian guppy (Poecilia reticulata). Behavioral Ecology, 23: 195-200.

Jirotkul, M. 1999. Operational sex ratio influences female preference and male-male competition in guppies. Animal Behaviour, 58: 287-294. 
Jirotkul, M. 2000. Male trait distribution determined alternative mating tactics in guppies. Journal of Fish Biology, 56: 1427-1434.

Johansson, B.G. and Jones, T.M. 2007. The role of chemical communication in mate choice. Biological Reviews, 82: 265-289.

Johansson, J., Turesson, H. and Persson, A. 2004. Active selection for large guppies, Poecilia reticulata, by the pike cichlid, Crenicichla saxatilis. Oikos, 105: 595-605.

Johnson, K.V.-A., Aplin, L.M., Cole, E.F., Farine, D.R., Firth, J.A., Patrick, S.C. and Sheldon, B.C. 2017. Male great tits assort by personality during the breeding season. Animal Behaviour, 128: 21-32.

Jones, K.A., Croft, D.P., Ramnarine, I.W. and Godin, J.-G.J. 2010. Size-assortative shoaling in the guppy (Poecilia reticulata): the role of active choice. Ethology, 116: 147-154.

Jones, K. A. and Godin, J.-G.J. 2010. Are fast explorers slow reactors? Linking personality type and anti-predator behaviour. Proceedings of the Royal Society B, 277: 625-632.

Kang, C., Sherratt, T.N., Kim, Y.E., Shin, Y., Moon, J., Song, U., Kang, J.Y., Kim, K. and Jang, Y. 2017. Differential predation drives the geographical divergence in multiple traits in aposematic frogs. Behavioral Ecology, 28: 1122-1130.

Kats, L.B. and Dill, L.M. 1998. The scent of death: Chemosensory assessment of predation risk by prey animals. Écoscience, 5: 361-394.

Kelley, J.L., Graves, J.A. and Magurran, A.E. 1999. Familiarity breeds contempt in guppies. Nature, 401: 661-662. 
Kelley, J.L. and Magurran, A.E. 2003. Effects of relaxed predation pressure on visual predator recognition in the guppy. Behavioral Ecology and Sociobiology, 54: 225-232.

Kelley, J.L., Morrell, L.J., Inskip, C., Krause, J. and Croft, D.P. 2011. Predation risk shapes social networks in fission-fusion populations. PLOS ONE, 6: e24280.

Kelly, C.D., Godin, J.-G.J. and Wright, J.M. 1999. Geographical variation in multiple paternity in natural populations of the guppy (Poecilia reticulata). Proceedings of the Royal Society B, 266: 2403-2408.

Kelly, C.D. and Godin, J.-G.J. 2001. Predation risk reduces male-male sexual competition in the Trinidadian guppy (Poecilia reticulata). Behavioral Ecology and Sociobiology, 51: 95100.

Kerth, G. and Konig, B. 1999. Fission, fusion and nonrandom associations in female Bechstein's bats (Myotis bechsteinii). Behaviour, 136: 1187- 1202.

Kerth, G., Perony, N. and Schweitzer, F. 2011. Bats are able to maintain long-term social relationships despite the high fission-fusion dynamics of their groups. Proceedings of the Royal Society B, 278: 2761-2767.

Kimbell, H.S. and Morrell, L.J. 2015. Turbidity influences individual and group level responses to predation in guppies, Poecilia reticulata. Animal Behaviour, 103: 179-185.

Kimbell, H.S. and Morrell, L.J. 2016. Turbidity weakens selection for assortment in body size in groups. Behavioral Ecology, 27: 545-552. 
King, A.J. and Cowlishaw, G. 2007. When to use social information: the advantage of large group size in individual decision making. Biology Letters, 3: 137-139.

Kokko, H., Brooks, R., Jennions, M.D. and Morley, J. 2003. The evolution of mate choice and mating biases. Proceeding of the Royal Society of London B, 270: 653-664.

Kolluru, G.R. and Grether, G.F. 2005. The effects of resource availability on alternative mating tactics in guppies (Poecilia reticulata). Behavioral Ecology, 16: 294-300.

Kolluru, G.R., Grether, G.F. and Contreras, H. 2007. Environmental and genetic influences on mating strategies along a replicated food availability gradient in guppies (Poecilia reticulata). Behavioral Ecology and Sociobiology, 61: 689-701.

Kotiaho, J., Alatalo, R.V., Mappes, J., Parri, S. and Rivero, A. 1998. Male mating success and risk of predation in a wolf spider: A balance between sexual and natural selection? Journal of Animal Ecology, 67: 287-291.

Krakauer, D.C. 1995. Groups confuse predators by exploiting perceptual bottlenecks: a connectionist model of the confusion effect. Behavioral Ecology and Sociobiology, 36: 421-429.

Krause, J. and Godin, J.-G.J. 1994. Shoal choice in the banded killifish (Fundulus diaphanus, Teleostei, Cyprinodontidae): effects of predation risk, fish size, species composition and size of shoals. Ethology, 98: 128-136.

Krause, J., Godin, J.-G.J. and Brown, D. 1996. Size-assortativeness in multi-species fish shoals. Journal of Fish Biology, 49: 221-225. 
Krause, J. and Ruxton, G.D. 2002. Living in Groups. Oxford University Press, Oxford.

Krebs, J.M. and Bell, S.S. 2012. Risk of predation reflects variation in the reproductive strategy of a dominant forage fish in mangrove tidal tributaries. Oecologia, 168: 737-748.

Krebs, J.R. and Davies, N.B. 1993. An Introduction to Behavioural Ecology. $3^{\text {rd }}$ Edition. Blackwell Publishing.

Landeau, L. and Terborgh, J. 1986. Oddity and the "confusion effect" in predation. Animal Behaviour, 34: 1372-1380.

Lawrence, B.J. and Smith, R.J.F. 1989. Behavioral response of solitary fathead minnows, Pimephales promelas, to alarm substance. Journal of Chemical Ecology, 15: 209-219.

Lazarus, J. 1979. The early warning function of flocking in birds: an experimental study with captive quelea. Animal Behaviour, 27: 855-865.

Lewis, S., South, A., Burns, R. and Al-Wathiqui, N. 2012. Nuptial gifts. Current Biology, 21: R644-R645.

Liley, N.R. 1966. Ethological isolating mechanisms in four sympatric species of Poeciliid fishes. Behaviour (Supplement), 14: 1-197.

Lima, S.L. 1995. Back to the basics of anti-predatory vigilance: the group-size effect. Animal Behaviour, 49: 11-20.

Lima, S.L. 2009. Predators and the breeding bird: behavioral and reproductive flexibility under the risk of predation. Biological Reviews, 84: 485-513. 
Lima, S.L. and Bednekoff, P.A. 1999. Temporal variation in danger drives antipredator behavior: the predation risk allocation hypothesis. American Naturalist, 153: 649-659.

Lima, S.L. and L.M. Dill. 1990. Behavioral decisions made under the risk of predation: a review and prospectus. Canadian Journal of Zoology, 68: 619-640.

Lindström, L., Ahtiainen, J.J., Mappes, J., Kotiaho, J.S., Lyytinen, A. and Alatalo, R.V. 2006. Negatively condition dependent predation cost of a positively condition dependent sexual signalling. Journal of Evolutionary Biology, 19: 649-656.

Long, T.A.F., Pischedda, A., Stewart, A.D. and Rice, W.R. 2009. A cost of sexual attractiveness to high-fitness females. PLoS One, 7: e1000254.

Loyau, A., Blanchet, S., Van Laere, P., Clobert, J. and Danchin, E. 2012. When not to copy: female fruit flies used sophisticated public information to avoid mated males. Scientific Reports, 2:768.

Luyten, P.H. and Liley, N.R. 1985. Geographic variation in the sexual behaviour of the guppy, Poecilia reticulata (Peters). Behaviour, 95: 164-179.

Lynch, M. 1980. The evolution of cladoceran life histories. Quarterly Review of Biology, 55: $23-42$.

Maan, M.E., Seehausen, O. and van Alphen, J.J.M. 2010. Female mating preferences and male coloration covary with water transparency in a Lake Victoria cichlid fish. Biological Journal of the Linnean Society, 99: 398-406. 
Magellan, K., Pettersson, L.B. and Magurran, A.E. 2005. Quantifying male attractiveness and mating behaviour through phenotypic size manipulation in the Trinidadian guppy, Poecilia reticulata. Behavioral Ecology and Sociobiology, 58: 366-374.

Magnhagen, C. 1991. Predation risk as a cost of reproduction. Trends in Ecology and Evolution, 6: 183-186.

Magurran, A.E. 2005. Evolutionary Ecology: the Trinidadian Guppy. Oxford University Press, Oxford.

Magurran, A.E. and Seghers, B.H. 1990. Risk sensitive courtship in the guppy (Poecilia reticulata). Behaviour, 112: 194-201.

Magurran, A.E. and Seghers, B.H. 1991. Variation in schooling and aggression amongst guppy (Poecilia reticulata) populations in Trinidad. Behaviour, 118: 214-234.

Magurran, A.E. and Seghers, B.H. 1994a. Predator inspection behaviour covaries with schooling tendency amongst wild guppy, Poecilia reticulata, populations in Trinidad. Behaviour, 128: 121-134.

Magurran, A.E. and Seghers, B.H. 1994b. Sexual conflict as a consequence of ecology: evidence from guppy, Poecilia reticulata, populations in Trinidad. Proceedings of the Royal Society $B, 255:$ 31-36.

Magurran, A.E. and Seghers, B.H. 1994c. A cost of sexual harassment in the guppy, Poecilia reticulata. Proceedings of the Royal Society B, 258: 89-92. 
Magurran, A.E., Seghers, B.H., Shaw, P.W. and Carvalho, G.R. 1994. Schooling preferences for familiar fish in the guppy, Poecilia reticulata. Journal of Fish Biology, 45: 401-406.

Makowicz, A.M. and Schlupp, I. 2013. The direct costs of living in a sexually harassing environment. Animal Behaviour, 85: 569-577.

Mateos-Gonzalez, F., Quesada, J. and Senar, J.C. 2011. Sexy birds are superior at solving a foraging problem. Biology Letters, doi:10.1098/rsbl.2011.0163.

Matthews, I.M. and Magurran, A.E. 2000. Evidence for sperm transfer during sneaky mating in wild Trinidadian guppies. Journal of Fish Biology, 56: 1381-1386.

McCollum, S.A. and Leimberger, J.D. 1997. Predator-induced morphological changes in an amphibian: predation by dragonflies affects tadpole shape and color. Oecologia, 109: 615621.

McCracken, G.F. and Bradbury, J.W. 1981. Social organisation and kinship in the polygynous bat Phyllostomus hastatus. Behavioral Ecology and Sociobiology, 8: 11-34.

McKellar, A.E. and Hendry, A.P. 2011. Environmental factors influencing adult sex ratio in Poecilia reticulata: laboratory experiments. Journal of Fish Biology, 79: 937-953.

McKellar, A.E., Turcotte, M.M. and Hendry, A.P. 2009. Environmental factors influencing adult sex ratio in Poecilia reticulata. Oecologia, 159: 735-745.

McKinnon, L., Gilchrist, H.G. and Scribner, K.T. 2006. Genetic evidence for kin-based female social structure in common eiders (Somateria mollissima). Behavioral Ecology, 17: 614-621. 
McRobert, S.P. and Bradner, J. 1998. The influence of body coloration on shoaling preferences in fish. Animal Behaviour, 56: 611-615.

Mellan, D., Warren, A., Buckholt, M.A. and Matthews, L.M. 2014. Sexual history affects mating behavior and mate choice in the crayfish Orconectes limosus. Ethology, 120: 681692.

Milinski, M. 1977. Experiments on the selection by predators against spatial oddity of their prey. Zeitschrift für Tierpsychologie, 43: 311-325.

Mueller, H.C. 1971. Oddity and specific searching image more important than conspicuousness in prey selection. Nature, 233: 345-346.

Neff, B.D., Pitcher, T.E. and Ramnarine, I.W. 2008. Inter-population variation in multiple paternity and reproductive skew in the guppy. Molecular Ecology, 17: 2975-2984.

Oh, K.P. and Badyaev, A.V. 2010. Structure of social networks in a passerine bird: consequences for sexual selection and the evolution of mating strategies. American Naturalist, 176: E80-89.

Ojanguran, A.F. and Magurran, A.E. 2007. Male harassment reduces short-term female fitness in guppies. Behaviour, 144: 503-514.

Olson, V.A. and Owens, I.P.F. 1998. Costly sexual signals: are carotenoids rare, risky or required? Trends in Ecology and Evolution, 13: 510-514.

Ophir, A.G. and Galef, Jr, B.G. 2003. Female Japanese quail affiliate with live males that they have seen mate on video. Animal Behaviour, 66: 369-375. 
Orrock, J.L., Danielson, B.J. and Brinkerhoff, R.J. 2004. Rodent foraging is affected by indirect, but not direct, cues of predation risk. Behavioral Ecology, 15: 433-437.

Palumbi, S.R. 2001. Humans as the world's greatest evolutionary force. Science, 293: 1786-1790.

Paul, A. 2002. Sexual selection and mate choice. International Journal of Primatology, 23: 877-904.

Peluc, S.I., Sillett, T.S., Rotenberry, J.T. and Ghalambor, C.K. 2008. Adaptive phenotypic plasticity in an island songbird exposed to a novel predation risk. Behavioural Ecology, 19: 830-835.

Penry-Williams, I.L., Ioannou, C.C. and Taylor, M.I. 2018. The oddity effect drives prey choice but not necessarily attack time. Ethology, 124: 496-503.

Petranka, J.W., Katz, L.B. and Sih, A. 1987. Predator-prey interactions amongst fish and larval amphibians: use of chemical cues to detect predatory fish. Animal Behaviour, 35: 420-425.

Pettersson, L.B., Ramnarine, I.W., Becher, S.A., Mahabir, R. and Magurran, A.E. 2004. Sex ratio dynamics and fluctuating selection pressures in natural populations of the Trinidadian guppy, Poecilia reticulata. Behavioral Ecology and Sociobiology, 55: 461-468.

Pilastro, A. and Bisazza, A. 1999. Insemination frequency of two alternative male mating tactics in the guppy (Poecilia reticulata). Proceedings of the Royal Society B, 266: 18871891. 
Pilastro, A., Simonato, M., Bisazza, A. and Evans, J.P. 2004. Cryptic female preference for colorful males in guppies. Evolution, 58: 665-669.

Piyapong, C., Krause, J., Chapman, B.B., Ramnarine, I.W., Louca, V. and Croft, D.P. 2010. Sex matters: a social context to boldness in guppies (Poecilia reticulata). Behavioral Ecology, 21: 3-8.

Pocklington, R. and Dill, L.M. 1995. Predation on females or males: who pays for bright male traits? Animal Behaviour, 49: 1122-1124.

Procter, D.S., Moore, A.J. and Miller, C.W. 2012. The form of sexual selection arising from male-male competition depends on the presence of females in the social environment. Journal of Evolutionary Biology, 25: 803- 812.

R Core Team. 2018. R: A language and environment for statistical computing. R Foundation for Statistical Computing, Vienna, Austria. http://www.R-project.org/.

Relyea, R.A. 2003. How prey respond to combined predators: a review and an empirical test. Ecology, 84: 1827-1839.

Renan, S., Speyer, E., Ben-Nun, T., Ziv, A., Greenbaum, G., Templeton, A., Bar-David, S. and Bouskila, A. 2018. Fission-fusion social structure of a reintroduced ungulate: Implications for conservation. Biological Conservation, 222: 261-267.

Reznick, D., Butler IV, M.J. and Rodd, H. 2001. Life-history evolution in guppies. VII. The comparative ecology of high- and low-predation environments. American Naturalist, 157: 126-140. 
Reznick, D. and Endler, J.A. 1982. The impact of predation on life history evolution in Trinidadian guppies (Poecilia reticulata). Evolution, 36: 160-177.

Řežucha, R. and Reichard, M. 2014. The effect of social environment on alternative mating tactics in male Endler's guppy, Poecilia wingei. Animal Behaviour, 88: 195-202.

Řežucha, R. and Reichard, M. 2016. The association between personality traits, morphological traits and alternative mating behaviour in male Endler's guppies, Poecilia wingei. Ethology, 122: 456-467.

Richards, E.L., van Oosterhout, C. and Cable, J. 2010. Sex-specific shoaling differences affect parasite transmission in guppies. PLoS ONE, 5: e13285.

Riipi, M., Alatalo, R.V., Lindström, L. and Mappes, J. 2001. Multiple benefits of gregariousness cover detectability costs in aposematic aggregations. Nature, 413: 512514.

Rodgers, G.M., Downing, B. and Morrell, L.J. 2015. Prey body size mediates the predation risk associated with being "odd". Behavioral Ecology, 26: 242-246.

Rodgers, G.M., Kimbell, H. and Morrell, L.J. 2013. Mixed-phenotype grouping: the interaction between oddity and crypsis. Oecologia, 172: 59-68.

Rose, P.E. and Croft, D.P. 2015. The potential of social network analysis as a tool for the management of zoo animals. Animal Welfare, 24: 123-138.

Rosenthal, G.G. and Ryan, M.J. 2005. Assortative preferences for stripes in danios. Animal Behaviour, 70: 1063-1066. 
Ruckstuhl, K.E. and Neuhaus, P. 2002. Sexual segregation in ungulates: a comparative test of three hypotheses. Biological Review of the Cambridge Philosophical Society, 77: 77-96.

Russell, S.T., Kelley, J.L., Graves, J.A. and Magurran, A.E. 2004. Kin structure and shoal composition dynamics in the guppy, Poecilia reticulata. Oikos, 106: 520-526.

Ruxton, G.D. 2009. Non-visual crypsis: a review of the empirical evidence for camouflage to senses other than vision. Philosophical Transactions of the Royal Society B, 364: 549557.

Ruxton, G.D., Allen, W.L., Sherratt, T.N. and Speed, M.P. 2018. Avoiding Attack: The Evolutionary Ecology of Crypsis, Aposematism, and Mimicry. $2^{\text {nd }}$ Edition. Oxford University Press.

Saaristo, M., McLennan, A., Johnstone, C.P., Clarke, B.O. and Wong, B.B.M. 2017. Impacts of the antidepressant fluoxetine on the anti-predator behaviours of wild guppies (Poecilia reticulata). Aquatic Toxicology, 183: 38-45.

Santos, R.G., Pinheiro, H.T., Martins, A.S., Riul, P., Bruno, S.C., Janzen, F.J. and loannou, C.C. 2016. The anti-predator role of within-nest emergence synchrony in sea turtle hatchlings. Proceedings of the Royal Society B, 283: 20160697.

Scarponi, V., Chowdhury, D. and Godin, J.-G.J. 2015. Male mating history influences female mate choice in the Trinidadian guppy (Poecilia reticulata). Ethology, 121: 10911103. 
Scarponi, V. and Godin, J.-G.J. 2018. Female assessment of male functional fertility during mate choice in a promiscuous fish. Ethology, 124: 196-208.

Schlupp, I. 2018. Male mate choice in livebearing fishes: an overview. Current Zoology, 64: 393-403.

Schlupp, I., Marler, C. and Ryan, M.J. 1994. Benefit to sailfin mollies mating with heterospecific females. Science, 263: 373-374.

Schmidt, K.A., Dall, S.R.X. and van Gils, J.A. 2010. The ecology of information: an overview on the ecological significance of making informed decisions. Oikos, 119: 304-316.

Schneider, C. A., Rasband, W.S. and Eliceiri, K.W. 2012. NIH Image to ImageJ: 25 years of image analysis. Nature Methods, 9: 671-675.

Schoeppner, N.M. and Relyea, R.A. 2009. Interpreting the smells of predation: how alarm cues and kairomones induce different prey defences. Functional Ecology, 23: 1114-1121.

Schuett, W., Tregenza, T. and Dall, S.R.X. 2010. Sexual selection and animal personality. Biological Reviews, 85: 217- 246.

Segami Marzel, J.C., Rudh, A., Rogell, B., Ödeen, A., Løvlie, H., Rosher, C. and Qvarnström, A. 2017. Cryptic female strawberry poison dart frogs experience elevated predation risk when associating with an aposematic partner. Ecology and Evolution, 7: 744-750.

Seghers, B.H. 1974. Schooling behavior in the guppy (Poecilia reticulata): An evolutionary response to predation. Evolution, 28: 486-489. 
Senar, J. C. and Escobar, D. 2002. Carotenoid derived plumage coloration in the siskin Carduelis spinus is related to foraging ability. Avian Science, 2: 19-24.

Shenoy, K. 2012. Environmentally realistic exposure to the herbicide Atrazine alters some sexually selected traits in male guppies. PLoS One, 7: e30611.

Sigurjónsdottir, H. and Haraldsson, H. 2019. Significance of group composition for the welfare of pastured horses. Animals, 9: 14.

Sih, A. 1994. Predation risk and the evolutionary ecology of reproductive behaviour. Journal of Fish Biology, 45: 111-130.

Sih, A. 2013. Understanding variation in behavioural responses to human-induced rapid environmental change: a conceptual overview. Animal Behaviour, 85: 1077-1088.

Sih, A., Bell, A. and Johnson, J.C. 2004. Behavioral syndromes: an ecological and evolutionary overview. Trends in Ecology and Evolution, 19: 372-378.

Sih, A., Cote, J., Evans, M., Fogarty, S. and Pruitt, J. 2012. Ecological implications of behavioural syndromes. Ecology Letters, 15: 278-289.

Sih, A. and Del Giudice, M. 2012. Linking behavioural syndromes and cognition: a behavioural ecology perspective. Philosophical Transactions of the Royal Society $B, 367$ : $2762-2772$.

Sih, A., Ferrari, M.C.O and Harris, D.J. 2011. Evolution and behavioural responses to human-induced rapid environmental change. Evolutionary Applications, 4: 367-687. 
Sih, A., Ziemba, R. and Harding, K.C. 2000. New insights on how temporal variation in predation risk shapes prey behavior. Trends in Ecology and Evolution, 15: 3-4.

Silk, J.B. 2002. Kin selection in primate groups. International Journal of Primatology, 23: 849-875.

Silk, J.B. 2007. The adaptive value of sociality in mammalian groups. Philosophical Transactions of the Royal Society B, 362: 539-559.

Silk, J.B., Beehner, J.C., Bergman, T.J., Crockford, C., Engh, A.L., Moscovice, L.R., Wittig, R.M., Seyfarth, R.M. and Cheney, D.L. 2010. Strong and consistent social bonds enhance the longevity of female baboons. Current Biology, 20: 1359-1361.

Silk, M.J., Croft, D.P., Delahay, R.J., Hodgson, D.J., Weber, N., Boots, M. and McDonald, R.A. 2017. The application of statistical network models in disease research. Methods in Ecology and Evolution, 8: 1026-1041.

Silk, M. J., Croft, D.P., Tregenza, T. and Bearhop, S. 2014. The importance of fission-fusion social group dynamics in birds. Ibis, 156: 701-715.

Stamps, J. and Groothuis, T.G.G. 2010. The development of animal personality: relevance, concepts and perspectives. Biological Reviews, 85: 301-325.

Stanley, C.R., Williams, H.L. and Preziosi, R.F. 2018. Female clustering in cockroach aggregations- A case of social niche construction? Ethology, 124: 706-718.

Sterck, E.H.M., Watts, D.P. and van Schaik, C.P. 1997. The evolution of female social relationships in nonhuman primates. Behavioral Ecology and Sociobiology. 41: 291-309. 
Stevens, M. and Merilaita, S. 2009. Animal camouflage: current issues and new perspectives. Philosophical Transactions of the Royal Society B, 364: 423-427.

Stroeymeyt, N., Grasse, A.V., Crespi, A., Mersch, D.P., Cremer, S. and Keller, L. 2018. Social network plasticity decreases disease transmission in a eusocial insect. Science, 362: 941945.

Sueur, C., Petit, O. and Deneubourg, J.L. 2010. Short-term group fission processes in macaques: a social networking approach. Journal of Experimental Biology, 213: 13381346.

Theodorakis, C.W. 1989. Size segregation and the effects of oddity on predation risk in minnow schools. Animal Behaviour, 38: 496-502.

Thomas, M.L. 2011. Detection of female mating status using chemical signals and cues. Biological Reviews, 86: 1-13.

Tóth, Z. and Griggio, M. 2011. Leaders are more attractive: Birds with bigger yellow breast patches are followed by more group-mates in foraging groups. PLos One, 6: e26605.

Turner, G.F. and Pitcher, T.J. 1986. Attack abatement: a model for group protection by combined avoidance and dilution. American Naturalist, 128: 228-240.

Tyler, F., Fisher, D., d'Ettorre, P., Rodriguez-Munõz, R. and Tregenza, T. 2015. Chemical cues mediate species recognition in field crickets. Frontiers in Ecology and Evolution, 3: 48. 
Vahed, K. 1998. The function of nuptial feeding in insects: a review of empirical studies. Biological Reviews, 73: 43-78.

Valone, T.J. 2007. From eavesdropping on performance to copying the behavior of others: a review of public information use. Behavioral Ecology and Sociobiology, 62: 1-14.

Verdolin, J.L. 2006. Meta-analysis of foraging and predation risk trade-offs in terrestrial systems. Behavioral Ecology and Sociobiology, 60: 457-464.

Ward, A. and Webster, M. 2016. Sociality: The Behaviour of Group-Living Animals. Springer International, Switzerland.

Wedell, N., Gage, M.J.G. and Parker, G.A. 2002. Sperm competition, male prudence and sperm-limited females. Trends in Ecology and Evolution, 17: 313-320.

Werner, E.E., Gilliam, J.F., Hall, D.J. and Mittelbach, G.G. 1983. An experimental test of the effects of predation risk on habitat use in fish. Ecology, 64: 1540-1548.

Wilkinson, G.S., Carter, G., Bohn, K.M., Caspers, B., Chaverri, G., Farine, D., Günther, L., Kerth, G., Knörnschild, M., Mayer, F., Nagy, M., Ortega, J. and Patriquin, K. 2019. Kinship, association and social complexity in bats. Behavioral Ecology and Sociobiology, 73: 7.

Wisenden, B.D. 2000. Olfactory assessment of predation risk in the aquatic environment. Philosophical Transactions of the Royal Society of London B, 355: 1205-1208.

Witte, K. and Massmann, R. 2003. Female sailfin mollies, Poecilia latipinna, remember males and copy the choice of others after 1 day. Animal Behaviour, 65: 1151-1159. 
Witte, K. and Ryan, M.J. 2002. Male choice copying in the sailfin molly, Poecilia latipinna, in the wild. Animal Behaviour, 63: 943-949.

Wong, B.B.M. and Candolin, U. 2005. How is female mate choice affected by male competition? Biological Reviews, 80: 559-571.

Zanette, L.Y., White, A.F., Allen, M.C. and Clinchy, M. 2011. Perceived predation risk reduces the number of offspring songbirds produce per year. Science, 334: 1398-1401.

Zhang, J.-L., Zhang, C.-N., Li, E.-C., Jin, M.-M., Huang, M.-X., Cui, W., Lin, Y.-Y. and Shi, Y.J. 2019. Triphenyltin exposure affects mating behaviors and attractiveness to females during mating in male guppies (Poecilia reticulata). Ecotoxicology and Environmental Safety, 169: 76-84 


\section{Appendices}


Appendix 1. Confirmation that discarding trials where significant (>80\%) side bias is observed does not significantly alter the type I error rate (including R code).

As highlighted in Section 2.3, following previous convention (established in Schlupp et al. 1994), any fish that spent more than $80 \%$ of its total shoaling time (i.e. the time that it was in one or other of the two association zones) on one side of the tank across the two preference tests was considered to have exhibited a side bias and was therefore discounted from further analysis. In order to ensure that this criterion did not asversely affect the data and subsequent analysis, a simulation comparison was carried out based on the null hypothesis $(\mathrm{NH})$ that focal male guppies do not express a significant preference for more sexually attractive male conspecifics. The simulation comparison considered the sampling distribution when i) all trials were used and ii) only trials without a consistent side bias were used. I also considered whether the difference in sampling distribution (if observed) affected the type I error rate - rejection of a true null hypothesis.

Preference for attractive males was measured as:

$$
\frac{x_{1 L}+x_{2 R}}{x_{1 T}+x_{2 T}} \text { or } \frac{x_{1 R}+x_{2 L}}{x_{1 T}+x_{2 T}}
$$

depending on whether attractive males were in the left end compartment or the right end compartment of the experimental apparatus in the first trial (as stimulus males are switched before the second trial, any male on the left during the first trial would be on the right during the second trial and vice versa). $x_{1 R}$ is the absolute length of time the focal fish spends in the right preference zone in the first trial, and $x_{2 L}$ is the absolute length of 
time the focal fish spends in the left preference zone in the second test, and $x_{1 T}$ is the total time spent in either preference zone in trial 1.

Data were excluded if a consistent side bias was observed, represented as:

$$
\frac{x_{1 L}+x_{2 L}}{x_{1 T}+x_{2 T}}>0.8 \text { or } \frac{x_{1 R}+x_{2 R}}{x_{1 T}+x_{2 T}}>0.8
$$

$1,000,000$ rows of data were created where $x_{1 \top}$ and $x_{2 T}$ are drawn from a uniform distribution between 0 and 1 . Once $x_{1 \top}$ and $x_{2 T}$ are set, random fractions (again drawn from a uniform distribution between 0 and 1) of these totals are set to be $x_{1 L}$ and $x_{2 L}$ from which $\mathrm{x}_{1 \mathrm{R}}\left(=\mathrm{x}_{1 \mathrm{~T}}-\mathrm{x}_{1 \mathrm{~L}}\right)$ and $\mathrm{x}_{2 \mathrm{R}}\left(=\mathrm{x}_{2 \mathrm{~T}}-\mathrm{x}_{2 \mathrm{~L}}\right)$ were calculated. Since it was now known in a trial $\left(x_{1 L}, x_{1 R}, x_{1 T}, x_{2} L, x_{2 R}, x_{2 T}\right)$ when the $N H$ is true (no preference for attractive males), preferences were calculated and those showing chance left or right biases could be discarded.

On average $21.5 \%$ of observations were excluded from the full data set because of consistent left or right biases. The sample distributions of preference (grey) and restricted preference (pink), in which I omitted the extremes ( $>0.8$ left or right), are indeed different, but not radically so (see figure below). More restricted preference measures are closer to 0.5 (lower variance) because some extreme preference measures (albeit for sides, not attractiveness) were excluded. This shows that while there is some effect to these criteria, the selection criterion has not substantially altered the sample distribution of preference. 


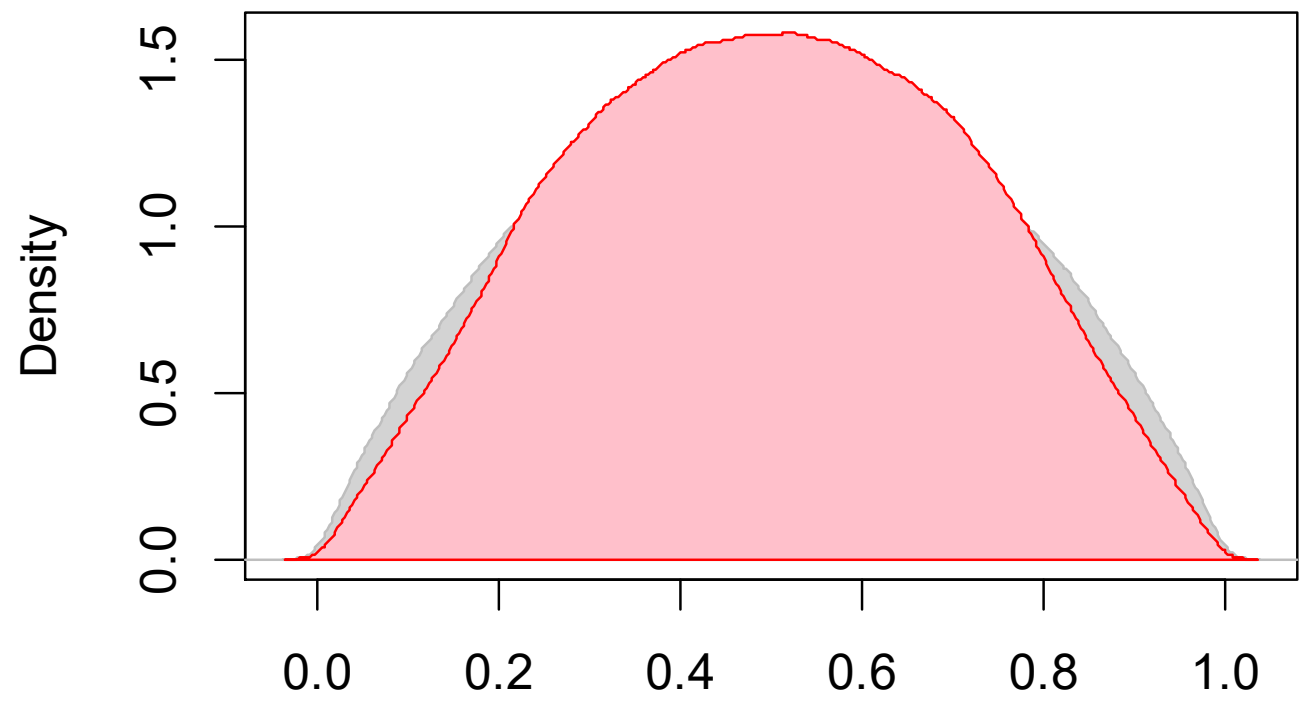

Sample preference distributions based on the full simulated data (grey) and the restricted data (pink- after consistent side bias removed)

Next, the type I error rate (the probability of obtaining a statistically significant result when in fact the $\mathrm{NH}$ is true) was evaluated from a standard experiment in which the preferences of 30 focal fish were observed. The million data rows were sampled 10,000 times and each time 30 observations of preference were selected (either from all the data, or only the data where no left/right bias was shown). In each of these 10,000 cases, a one sample t-test was conducted to evaluate whether the null hypothesis that the population mean preference is 0.5 could be rejected.

The type I error rate was estimated as 0.0495 when all the preference data were used and a significance cut-off of 0.05 was assumed. Likewise, the type I error rate was estimated to be 0.0507 when the restricted preference data were used. Therefore, in 
both cases, the estimated type I error rate is as expected (i.e. close to the significance threshold that was set). Overall, while the sampling distribution of preference differs slightly by excluding observations with consistent left or right biases, it has little to no effect on the type I error rate. 


\section{R Code for the simulation}

$\mathrm{n}<-1000000$; crit $=0.8$

xT1<- numeric(n); xL1<- numeric(n); xR1<- numeric(n)

$x T 2<-$ numeric(n); $x \mathrm{~L} 2<-$ numeric(n); $x R 2<-$ numeric(n)

$x T 1<-\operatorname{runif}(n) ; x L 1<-\operatorname{runif}(n) * x T 1 ; x R 1<-x T 1-x L 1$

$x T 2<-$ runif(n); $x \mathrm{~L} 2<-\operatorname{runif}(\mathrm{n}) * x T 2 ; x R 2<-x T 2-x \mathrm{~L} 2$

$\mathrm{pL}<-(x \mathrm{~L} 1+\mathrm{xL} 2) /(x \mathrm{~T} 1+x \mathrm{~T} 2) ; \mathrm{pR}<-(x R 1+x R 2) /(x T 1+x T 2)$

$z \mathrm{~L}<-$ ifelse $((\mathrm{pL}>\mathrm{crit}), 0,1) ; \mathrm{zR}<-$ ifelse $((\mathrm{pR}>\mathrm{crit}), 0,1)$

$z<-$ ifelse $(z L+z R<2,0,1) ; r<-$ sample $(0: 1, n$, replace $=T)$

$d<-$ data.frame( $x T 1, x L 1, x R 1, x T 2, x L 2, x R 2, z L, z R, z, r)$

\# Calculates distributions of preferences based on entire data and subset with no strong bias

ds $<-$ subset $(d, d \$ z>0)$

pAll<- ifelse $((d \$ r==0),(d \$ x R 1+d \$ x L 2) /(d \$ x T 1+d \$ x T 2),(d \$ x L 1+d \$ x R 2) /(d \$ x T 1+d \$ x T 2))$

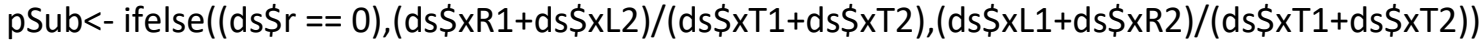

dens2<- density(na.omit(pSub))

plot(dens2, type="n")

dens1<- density(na.omit(pAll))

polygon(dens1, col="lightgray", border="gray")

polygon(dens2, col="pink", border="red")

\# Does one sample t test 10,000 times based on 30 data points and each time calculates $p$-value d\$pAll<- pAll

ds\$pSub $<-p S u b$

nt $<-10000$

v1<- numeric(nt)

v2<- numeric(nt)

for (i in 1:nt) \{

subd <- d[sample(nrow(d), 30), ]\$pAll

stest<- t.test(subd, mu=0.5)

v1[i]<- stest\$p.value

subds<- ds[sample(nrow(ds), 30), ]\$psub

stest<- t.test(subds, mu=0.5)

v2[i]<- stest\$p.value

\}

sum $(v 1<0.05)$

$\operatorname{sum}(v 2<0.05)$ 
Appendix 2. Confirmation that the total time focal males were observed did not significantly differ among the 3 different populations recorded.

a) Mean and standard error of total time observed for focal males from the 3 different rivers

\begin{tabular}{|c|c|c|c|}
\hline & Quaré & Upper Aripo & Tunapuna \\
& (high-predation) & (low-predation) & (low-predation) \\
\hline Time Observed (sec) & 74.07 & 73.97 & 78.38 \\
\hline Standard Error & \pm 22.59 & \pm 8.93 & \pm 14.31 \\
\hline
\end{tabular}

b) Results of the ANOVA log (Time) as.factor(Population), showing that there was no significant difference in the total time focal males were observed for across the 3 different rivers. Time was log-transformed to improve normality and homogeneity of residuals.

\begin{tabular}{|c|c|c|c|c|c|}
\hline & DF & Sum Sq (Type 1) & Mean Sq & F & Pr (>F) \\
\hline Population & 2 & 0.426 & 0.213 & 0.421 & 0.658 \\
\hline Residuals & 61 & 30.905 & 0.507 & & \\
\hline
\end{tabular}


Appendix 3. Results of paired t-tests comparing the mean time spent shoaling (within one of the two preference zones) by focal females in Presentation 1 and Presentation 2 for both populations tested (Tunapuna= low-predation; Quaré= high-predation). $\mathrm{p}$ values indicate that there was no significant difference in the amount of time spent shoaling in Presentation 1 and Presentation 2 for any treatment.

\begin{tabular}{|c|c|c|c|c|c|c|}
\hline Population & Stimulus & Presentation 1 & Presentation 2 & $\mathbf{t}$ & d.f. & $\mathbf{p}$ \\
& Type & $\begin{array}{c}\text { Mean Shoaling } \\
\text { Time (secs) }\end{array}$ & $\begin{array}{c}\text { Mean Shoaling } \\
\text { Time (secs) }\end{array}$ & & & \\
\hline \multirow{2}{*}{$\begin{array}{c}\text { Tunapuna } \\
\text { River }\end{array}$} & Consistent & 1083.50 & 1083.86 & -0.035 & 21 & 0.973 \\
\cline { 2 - 7 } & Increased & 1104.25 & 1086.50 & 1.469 & 19 & 0.159 \\
\hline \multirow{2}{*}{ Quaré } & Consistent & 1069.95 & 1090.36 & -1.687 & 21 & 0.106 \\
\hline River & Increased & 1078.76 & 1073.82 & 0.288 & 16 & 0.777 \\
\cline { 2 - 8 } & & & & & & \\
\hline
\end{tabular}


Appendix 4. Results of paired t-tests to test the null hypothesis that there was no significant difference in (a) body length or (b) coloration between the focal and stimulus males, and also between the two sets of stimulus males (more and less attractive). All significant values indicated in boldface. In all cases more and less attractive stimulus males were significantly different from one another, and focal males were significantly different from the stimulus males of the opposite type to themselves (i.e. a less attractive focal male was significantly different from the more attractive stimulus males but was not significantly different from the less attractive stimulus males).

(a) Body length

\begin{tabular}{|c|c|c|c|c|c|}
\hline & & & \multicolumn{3}{|c|}{ Paired t-test results } \\
\hline Population & $\begin{array}{c}\text { Focal Male } \\
\text { Type }\end{array}$ & Cue type & Focal- Less & Focal-More & More-Less \\
\hline \multirow{4}{*}{$\begin{array}{l}\text { Tunapuna } \\
\text { (low- } \\
\text { predation) }\end{array}$} & \multirow{2}{*}{$\begin{array}{l}\text { Less } \\
\text { Attractive }\end{array}$} & Control & $\begin{array}{c}\mathrm{t}=0.39, \mathrm{df}=23 \\
\mathrm{p}=0.700\end{array}$ & $\begin{array}{c}t=-8.96, d f=23 \\
p<0.001\end{array}$ & $\begin{array}{c}t=8.96, d f=23 \\
p<0.001\end{array}$ \\
\hline & & Cue & $\begin{array}{c}t=-1.15, d f=22 \\
p=0.263\end{array}$ & $\begin{array}{c}t=-13.99, d f=22, \\
p<0.001\end{array}$ & $\begin{array}{c}t=12.31, d f=22 \\
p<0.001\end{array}$ \\
\hline & \multirow{2}{*}{$\begin{array}{c}\text { More } \\
\text { Attractive }\end{array}$} & Control & $\begin{array}{c}t=19.19, d f=21, \\
p<0.001\end{array}$ & $\begin{array}{c}t=0.77, d f=21, \\
p=0.447\end{array}$ & $\begin{array}{c}\mathrm{t}=12.86, \mathrm{df}=21 \\
\mathrm{p}<0.001\end{array}$ \\
\hline & & Cue & $\begin{array}{c}t=15.41, d f=22, \\
p<0.001\end{array}$ & $\begin{array}{c}\mathrm{t}=1.23, \mathrm{df}=22 \\
\mathrm{p}=0.230\end{array}$ & $\begin{array}{c}\mathrm{t}=14.00, \mathrm{df}=22 \\
p<0.001\end{array}$ \\
\hline \multirow{4}{*}{$\begin{array}{c}\text { Quaré } \\
\text { (high- } \\
\text { predation) }\end{array}$} & \multirow{2}{*}{$\begin{array}{c}\text { Less } \\
\text { Attractive }\end{array}$} & Control & $\begin{array}{c}t=-0.46, d f=22 \\
p=0.650\end{array}$ & $\begin{array}{c}t=-10.06, d f=22 \\
p<0.001\end{array}$ & $\begin{array}{c}t=8.94, d f=22 \\
p<0.001\end{array}$ \\
\hline & & Cue & $\begin{array}{c}t=-0.66, d f=22, \\
p=0.515\end{array}$ & $\begin{array}{c}t=-13.84, d f=22, \\
p<0.001\end{array}$ & $\begin{array}{c}t=11.31, d f=22 \\
p<0.001\end{array}$ \\
\hline & \multirow{2}{*}{$\begin{array}{c}\text { More } \\
\text { Attractive }\end{array}$} & Control & $\begin{array}{c}t=8.58, d f=19 \\
p=0.003\end{array}$ & $\begin{array}{c}t=-0.13, d f=19 \\
p=0.897\end{array}$ & $\begin{array}{c}t=9.39, d f=19 \\
p<0.001\end{array}$ \\
\hline & & Cue & $\begin{array}{c}t=5.91, d f=20, \\
p<0.001\end{array}$ & $\begin{array}{c}t=0.17, d f=20, \\
p=0.864\end{array}$ & $\begin{array}{c}\mathrm{t}=10.33, \mathrm{df}=20 \\
\mathrm{p}<0.001\end{array}$ \\
\hline
\end{tabular}


(b) Body coloration

\begin{tabular}{|c|c|c|c|c|c|}
\hline & & & \multicolumn{3}{|c|}{ Paired t-test results } \\
\hline Population & $\begin{array}{c}\text { Focal Male } \\
\text { Type }\end{array}$ & Cue type & Focal- Less & Focal-More & More-Less \\
\hline \multirow{4}{*}{$\begin{array}{l}\text { Tunapuna } \\
\text { (low- } \\
\text { predation) }\end{array}$} & \multirow{2}{*}{$\begin{array}{c}\text { Less } \\
\text { Attractive }\end{array}$} & Control & $\begin{array}{c}t=-0.42, d f=23, \\
p=0.676\end{array}$ & $\begin{array}{c}t=-8.14, d f=23, \\
p<0.001\end{array}$ & $\begin{array}{c}t=9.78, d f=23 \\
p<0.001\end{array}$ \\
\hline & & Cue & $\begin{array}{c}\mathrm{t}=-0.03, \mathrm{df}=22, \\
\mathrm{p}=0.976\end{array}$ & $\begin{array}{c}t=-5.66, d f=22, \\
p<0.001\end{array}$ & $\begin{array}{c}t=6.64, d f=22, \\
p<0.001\end{array}$ \\
\hline & \multirow{2}{*}{$\begin{array}{c}\text { More } \\
\text { Attractive }\end{array}$} & Control & $\begin{array}{c}t=5.28, d f=21, \\
p<0.001\end{array}$ & $\begin{array}{c}\mathrm{t}=-0.81, \mathrm{df}=21, \\
\mathrm{p}=0.425\end{array}$ & $\begin{array}{c}t=7.86, d f=21, \\
p<0.001\end{array}$ \\
\hline & & Cue & $\begin{array}{c}\mathrm{t}=5.70, \mathrm{df}=22, \\
p<0.001\end{array}$ & $\begin{array}{c}\mathrm{t}=-0.33, \mathrm{df}=22 \\
\mathrm{p}=0.746\end{array}$ & $\begin{array}{c}t=7.45, d f=22, \\
p<0.001\end{array}$ \\
\hline \multirow{4}{*}{$\begin{array}{c}\text { Quaré } \\
\text { (high- } \\
\text { predation) }\end{array}$} & \multirow{2}{*}{$\begin{array}{c}\text { Less } \\
\text { Attractive }\end{array}$} & Control & $\begin{array}{c}t=-0.71, d f=22, \\
p=0.484\end{array}$ & $\begin{array}{c}t=-6.42, d f=22, \\
p<0.001\end{array}$ & $\begin{array}{c}\mathrm{t}=6.89, \mathrm{df}=22, \\
\mathrm{p}<0.001\end{array}$ \\
\hline & & Cue & $\begin{array}{c}t=-0.79, d f=22, \\
p=0.438\end{array}$ & $\begin{array}{c}t=-9.23, d f=22 \\
p<0.001\end{array}$ & $\begin{array}{c}t=5.85, d f=22, \\
p<0.001\end{array}$ \\
\hline & \multirow{2}{*}{$\begin{array}{c}\text { More } \\
\text { Attractive }\end{array}$} & Control & $\begin{array}{c}t=3.47, d f=19, \\
p=0.003\end{array}$ & $\begin{array}{c}t=-0.56, d f=19, \\
p=0.581\end{array}$ & $\begin{array}{c}t=5.55, d f=19, \\
p<0.001\end{array}$ \\
\hline & & Cue & $\begin{array}{c}t=3.17, d f=20, \\
p=0.005\end{array}$ & $\begin{array}{c}\mathrm{t}=-1.65, \mathrm{df}=20 \\
\mathrm{p}=0.115\end{array}$ & $\begin{array}{c}\mathrm{t}=5.60, \mathrm{df}=20, \\
p<0.001\end{array}$ \\
\hline
\end{tabular}


Appendix Figure A1. Example of within-population variation in body length and coloration in male guppies collected from the Quaré River, Trinidad.

\section{Examples of Quaré River male guppies}
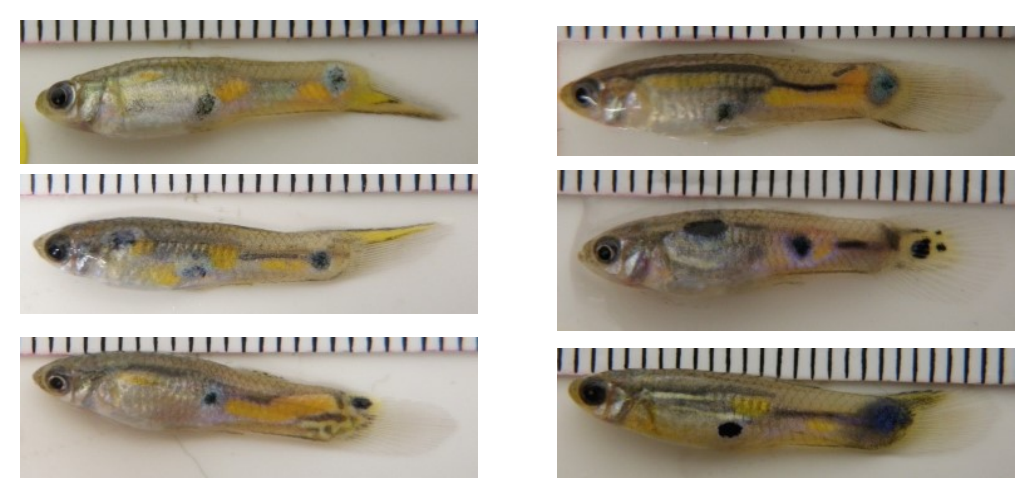

11111111111111111111111111110

11111111111||||||||
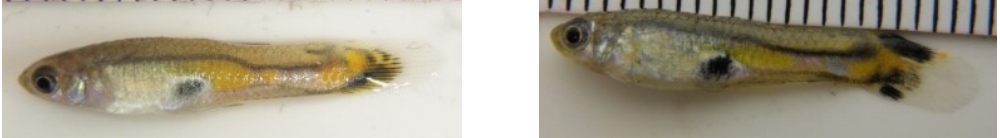
Appendix Figure A2. Map of the Northern Mountain Range region of Trinidad, with stars marking the sites used throughout this thesis (from left-right: Tunapuna, Arima, Upper Aripo, Quaré)

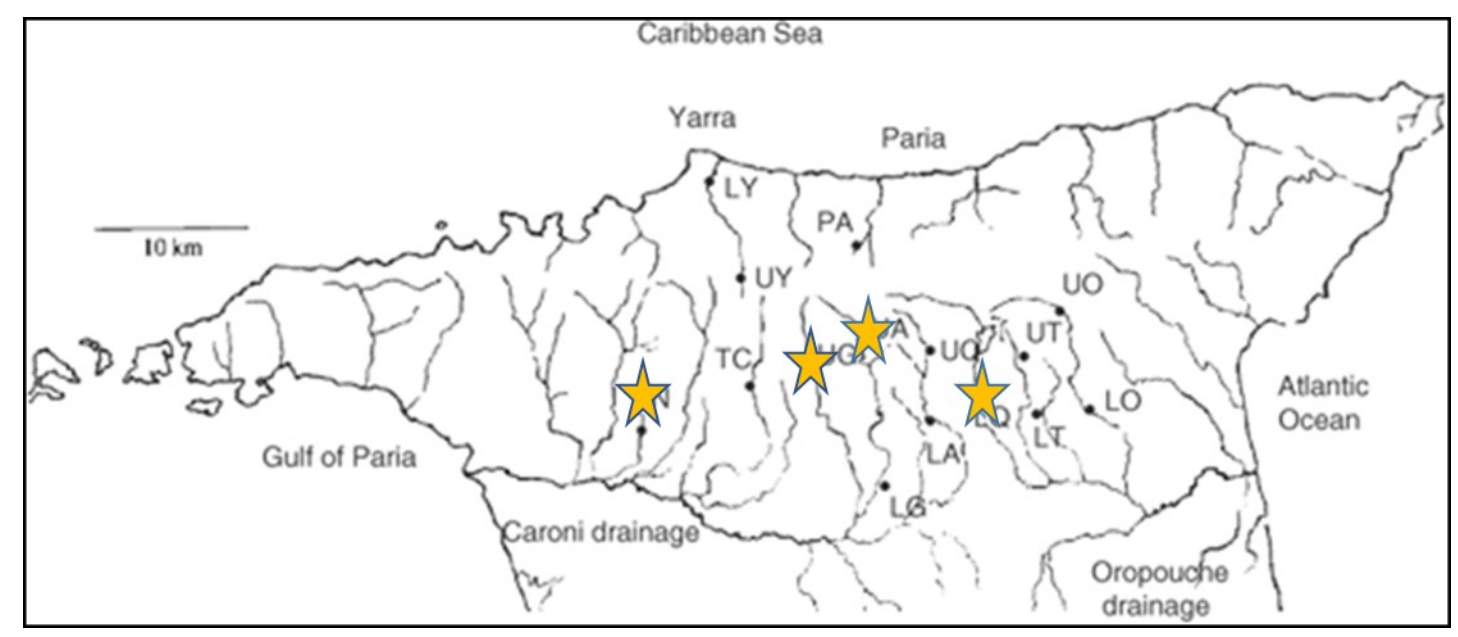

